\title{
1 Heavy metal uptake of near-shore benthic foraminifera during 2 multi-metal culturing experiments
}

\author{
3 Sarina Schmidt ${ }^{1}$, Edmund Charles Hathorne ${ }^{1}$, Joachim Schönfeld ${ }^{1}$ and Dieter Garbe- \\ 4 Schönberg ${ }^{2}$ \\ $5 \quad{ }^{1}$ GEOMAR Helmholtz Centre for Ocean Research Kiel, Wischhofstraße 1-3, 24148 Kiel, Germany \\ 6 2Institute of Geosciences, Kiel University, Ludewig-Meyn-Straße 10, 24118 Kiel, Germany \\ $7 \quad$ Correspondence to: Sarina Schmidt (sschmidt@geomar.de)
}

\begin{abstract}
Heavy metal pollution originating from anthropogenic sources, e.g., mining, industry and extensive land use, is increasing in many parts of the world and influences coastal marine environments for a long time. The elevated input of heavy metals into the marine system potentially affects the biota because of their toxicity, persistence and bioaccumulation. An emerging tool for environmental applications is the heavy metal incorporation into foraminiferal tests calcite, which facilitates monitoring of anthropogenic footprints on recent and past environmental systems. The aim of this study is to investigate whether the incorporation of heavy metals in foraminifera is a direct function of their concentration in seawater. Culturing experiments with a mixture of dissolved chromium $(\mathrm{Cr})$, manganese $(\mathrm{Mn})$, nickel $(\mathrm{Ni})$, copper $(\mathrm{Cu})$, zinc $(\mathrm{Zn})$, silver $(\mathrm{Ag})$, cadmium $(\mathrm{Cd})$, tin $(\mathrm{Sn})$, mercury $(\mathrm{Hg})$ and lead $(\mathrm{Pb})$ in artificial seawater were carried out over a wide concentration range to assess the uptake of heavy metals by the near-shore foraminiferal species Ammonia aomoriensis, Ammonia batava and Elphidium excavatum. Seawater analysis exhibited the increasing metal concentrations between culturing phases and revealed high metal concentrations in the beginning of the culturing phases due to the punctual metal addition. Furthermore, a loss of metals during the culturing process was discovered, which lead to a deviation between the expected and the actual concentrations of the metals in seawater. Laser ablation ICP-MS analysis of the newly formed calcite revealed species-specific differences in the incorporation of heavy metals. The foraminiferal calcite of all three species reveals a strong positive correlation with $\mathrm{Pb}$ and $\mathrm{Ag}$ concentrations in the culturing medium. Ammonia aomoriensis further showed a correlation with $\mathrm{Mn}$ and $\mathrm{Cu}$, A. batava with $\mathrm{Mn}$ and $\mathrm{Hg}$ and E. excavatum with $\mathrm{Cr}$ and $\mathrm{Ni}$, and partially also with $\mathrm{Hg}$. Zn, $\mathrm{Sn}$ and $\mathrm{Cd}$ showed no clear trend for the species studied, which may be caused by the little variation of these metals in seawater. Our calibrations and the calculated partition coefficients render A. aomoriensis, A. batava and E. excavatum as natural archives that enable the direct quantification of metals in polluted and pristine environments. This in turn allows monitoring of the ecosystem status of areas that are potentially under the threat of anthropogenic pollution in order to evaluate contemporary emission reduction measures.
\end{abstract}

\section{Introduction}

Particular heavy metals e.g., zinc $(\mathrm{Zn})$, iron $(\mathrm{Fe})$, molybdenum (Mo), cobalt $(\mathrm{Co})$ and copper $(\mathrm{Cu})$ serve as micronutrients (e.g., Hänsch and Mendel, 2009) for eukaryotic life and play an important role for metabolism, growth, reproduction and enzymatic activity of organisms (e.g., Martín-González et al., 2005; Gallego et al., 2007). Other metals like mercury $(\mathrm{Hg})$, on the other hand, are not known to have any positive effect on the body and are therefore believed to have a higher toxic potential (Jan et al., 2015). All these metals occur naturally in the environment as geogenic traces in soils, water, rocks and, consequently, in plants and animals. However, at higher 
concentrations, most heavy metals become toxic and have hazardous effects on marine biota (Stankovic et al., 2014). Furthermore, they are highly persistent in the marine environment and can be hardly degraded by organisms after the uptake of these metals into their system and cells (Flora et al., 2012; Kennish, 2019). Coastal environments act as natural catchments for anthropogenic pollutants because these areas are directly affected by industry, agriculture and urban runoff (e.g., Alloway, 2013; Julian, 2015; Tansel and Rafiuddin, 2016).

In marginal seas and coastal areas, benthic foraminifera are common, and they can be used as proxies for changing environmental parameters like water temperature (Mg/Ca; e.g., Nürnberg et al., 1995; 1996), salinity (Na/Ca; e.g., Wit et al., 2013, Bertlich et al., 2018), oxygen content or redox conditions ( $\mathrm{Mn} / \mathrm{Ca}$; Groeneveld and Filipsson, 2013b; Koho et al., 2015; 2017; Kotthoff et al., 2017; Petersen et al., 2018). Foraminifera take up heavy metals and incorporate them into their calcium carbonate shells during calcification (e.g., Boyle, 1981; Rosenthal et al., 1997; Dissard et al., 2010a; 2009; 2010b; Munsel et al., 2010; Nardelli et al., 2016; Frontalini et al., 2018a; 2018b; Titelboim et al., 2018; Smith et al., 2020). Moreover, foraminifera have a short life cycle (< 1 year; e.g., Haake, 1967; Boltovskoy and Lena, 1969; Wefer, 1976; Murray, 1992) and thus, react immediately to changing environmental conditions and contamination levels of the surrounding environment.

Species of the foraminiferal genera Elphidium and Ammonia are among the most abundant foraminiferal taxa in near-shore environments worldwide. They are found from subtidal water depths to the outer continental shelves (Murray, 1991). Furthermore, their calcite tests are often well preserved in the fossil record (Poignant et al., 2000; McGann, 2008; Xiang et al., 2008) and therefore provide the opportunity to assess past environmental conditions. The combination of all these properties make foraminifera, and especially Elphidium and Ammonia species, suitable indicators of anthropogenic pollution (e.g., Sen Gupta et al., 1996; Platon et al., 2005). As such, this group of organisms are excellent candidates for monitoring the spatial and temporal distribution of heavy metals in seawater to evaluate, for example, the effectiveness of contemporary measures of reducing emissions caused by anthropogenic inputs.

The majority of culturing studies on heavy metal incorporation into benthic foraminifera were designed to assess the influence and uptake of one particular metal, e.g., copper $(\mathrm{Cu})$ (De Nooijer et al., 2007), chromium $(\mathrm{Cr})$ (Remmelzwaal et al., 2019), lead (Pb) (Frontalini et al., 2015), zinc ( $\mathrm{Zn})$ (e.g., Smith et al., 2020), mercury (Hg) (Frontalini et al., 2018a) or cadmium (Cd) (Linshy et al., 2013). This approach is adequate to detail the effects on shell chemistry, growth or physiology. Only one study reported a culturing experiment with elevated levels of $\mathrm{Cu}$, Mn and Ni in the same culturing medium (Munsel et al., 2010). However, there is rarely only one but mostly a combination of several pollutants that occur in nearly all environments affected by heavy metal pollution (e.g., Mutwakil et al., 1997; Cang et al., 2004; Vlahogianni et al., 2007; Huang et al., 2011; Wokhe, 2015; Saha et al., 2017). How foraminifera incorporate and react to heavy metals when they are co-exposed to more than one metal at a time is less constrained to date. A mixture of different metals will lead to interactions, which may result in a more severe damage of tissue than exposure to each of them individually (Tchounwou et al., 2012). For example, a co-exposure to arsenic and cadmium causes a more distinct damage of human kidneys than only one of these elements (Nordberg et al., 2005). Furthermore, a chronic low-dose exposure to multiple elements can cause similar synergistic effects (e.g., Wang et al., 2008). It is therefore reasonable to assume that other organisms are likewise threatened more harmfully when exposed to several potentially toxic elements simultaneously. 
Here we present results from culturing studies with Ammonia aomoriensis, Elphidium exvacatum and Ammonia batava addressing the relationship of heavy metal concentration in seawater and foraminiferal tests. The partitioning factor between concentration of an element in the ambient seawater and the calcium carbonate of the foraminifers is constrained by determining both the dissolved metal concentrations in water and the metal contents of individual chambers of the foraminiferal shell that have been precipitated in the culturing medium. In particular, foraminifera were grown while exposed to a combination of ten different heavy metals, i.e., cadmium (Cd), copper $(\mathrm{Cu})$, chromium $(\mathrm{Cr})$, lead $(\mathrm{Pb})$, manganese $(\mathrm{Mn})$, mercury $(\mathrm{Hg})$, nickel $(\mathrm{Ni})$, silver $(\mathrm{Ag})$, tin $(\mathrm{Sn})$ and zinc $(\mathrm{Zn})$ over a range of concentrations that prevail in polluted near-shore environments today. These metals are the most common representatives of marine heavy metal pollution (Alve, 1995; Martinez-Colon et al., 2009). Once the carbonate/seawater metal partitioning coefficients are known, investigations of the chemistry of benthic foraminiferal shells offer a reliable method to monitor short-term changes in the chemistry and bioavailability of toxic elements in seawater.

\section{Material and Methods}

\subsection{Field sampling}

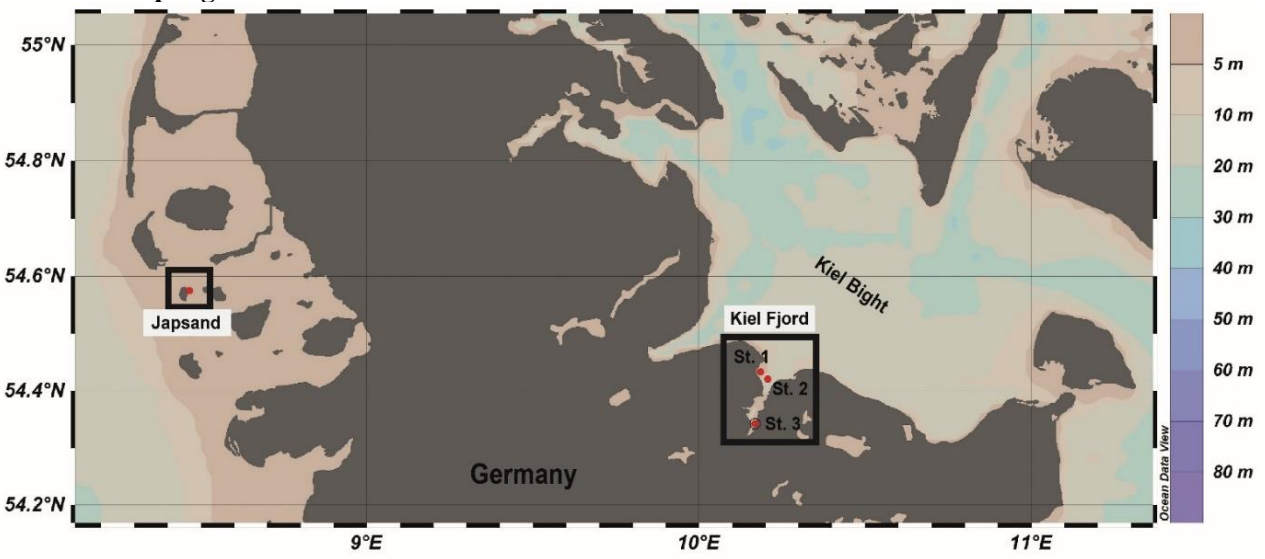

Figure 1: Location of the sampling stations in the North Sea (Japsand area) and in the Baltic Sea (Kiel Fjord, St.1 Strander Bucht, St. 2 Laboe, St. 3 Mönkeberg). The map was drawn with Ocean Data View (Schlitzer, 2016) on the basis of bathymetric data. Depth in $\mathrm{m}$ are indicated by the color code.

\subsubsection{North Sea, Japsand}

Living specimens of A. batava were collected at the barrier sand Japsand near Hallig Hooge in the German Wadden Sea in July 2019 at two stations (St. 1: $54^{\circ} 34.480^{`} \mathrm{~N}, 8^{\circ} 27.919^{`} \mathrm{E}$; St. $2: 54^{\circ} 34.491^{`} \mathrm{~N}, 8^{\circ} 27.895^{`} \mathrm{E}$ ) (Fig. 1). The sediment was a glacial till or Eemian clay at Station 1 and fine to medium sand at Station 2. The samples were recovered during low tide by scrapping off the uppermost centimetre of the surface sediment with a spoon made out of stainless steel. Natural seawater (NSW) with a salinity of 30.3 PSU was collected near the sites for further processing of the samples. Once back on Hallig Hooge, the sediment was washed with NSW through stacked sieves with a mesh size of 2000 and $63 \mu \mathrm{m}$. The $2000 \mu \mathrm{m}$ sieve was used to remove larger organisms and excess organic material (macroalgae, gastropods, lugworms etc.) that could induce anoxic conditions in the sediment 
https://doi.org/10.5194/bg-2021-158

Preprint. Discussion started: 12 August 2021

(c) Author(s) 2021. CC BY 4.0 License.

during transport and storage. The residue was stored in Emsa CLIP and CLOSE® boxes, sparged with air and some algae food was provided. Back in the laboratory at GEOMAR, the residue was stored at $8{ }^{\circ} \mathrm{C}$ in a fridge until culturing. These stock cultures were fed twice a week with Nannochloropsis concentrate (BlueBioTech) and water was partly exchanged with NSW from the sampling site once a week.

\subsubsection{Baltic Sea, Kiel Bight}

Living specimens of A. aomoriensis and E.excavatum were collected from different stations in Kiel Fjord, western

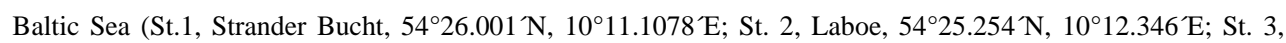
Mönkeberg, $54^{\circ} 20.752^{\prime} \mathrm{N}, 1^{\circ} 10.150^{\prime} \mathrm{E}$; water depth: $12.5 \mathrm{~m}, 12.3 \mathrm{~m}$ and $14.3 \mathrm{~m}$, respectively) in September and October 2019 with F.B. Polarfuchs and F.S. Alkor (Fig. 1). A Rumohr corer (inner diameter 55 mm) was used on F.B. Polarfuchs and 9 cores were taken (2 at St. 1 and 7 at St. 3). One core at both stations was used for foraminiferal assemblage analysis and the first $2 \mathrm{~cm}$ of the sediment from all other cores was collected in plastic containers with NSW from the site.

The sediment surface was nearly horizontal and comprised a $\sim 5 \mathrm{~mm}$ thick fluffy layer consisting of organic detritus of a dark brownish color. Mussels, worm burrows and plant debris was found. The sediment underneath the surface layer was a very fine mud. The redox boundary was shallower than 0.5 to $1 \mathrm{~cm}$ as indicated by the color turning black underneath this depth, and the sediment smelling of $\mathrm{H}_{2} \mathrm{~S}$.

On F.S. Alkor, a Reineck box corer was used $(200 \times 250 \mathrm{~cm})$ and 3 replicates at each station were taken (St. $1-$ $3)$. The first 1 to $2 \mathrm{~cm}$ of the sediment surface of the box core were scrapped off with a spoon made out of stainless steel and the material was stored in a plastic box with NSW from the location. Additional samples for foraminiferal assemblage analysis were taken at each station.

Back in the laboratory at GEOMAR, the samples were treated the same as Japsand samples from the North Sea. Artificial seawater (ASW, Tropic Marin) with a salinity of 30 PSU was used for washing and storage of the surface samples from Kiel Fjord. 

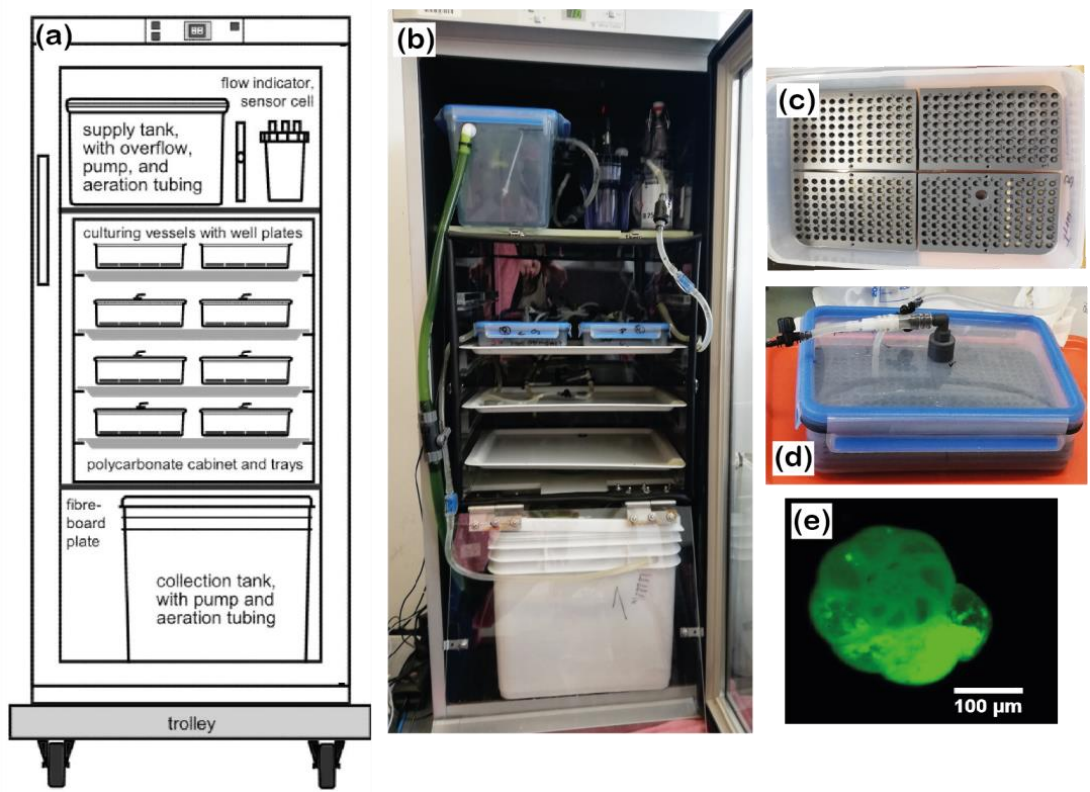

Figure 2: Culturing setup. a: conceptual draft (left, modified after Dagan et al., 2016) and b: assembly of the system (right). Tubing and hoses were omitted from the draft for clarity. c: a well plate with mounted specimens and sand, d: closed culturing vessel with well plates and conduits. e: with calcein stained foraminifer under a fluorescence microscope. The chambers formed in calcein are fluorescing brightly.

\subsubsection{Picking of the samples}

For extracting the foraminiferal specimens from the sediment, about $1 \mathrm{~cm}^{3}$ of the 63 to $2000 \mu \mathrm{m}$ size fraction was transferred to a petri dish. For maintaining optimal conditions for the foraminifera, the petri dish was filled with artificial seawater (ASW) with a salinity of 30 PSU. All living specimens were picked with a paint brush from this subsample and collected in a small petri dish of $55 \mathrm{~mm}$ diameter with ASW. The procedure was repeated until the whole sample residue was screened. Only specimens with a glossy, transparent and undamaged test were chosen. Furthermore, only individuals with the cytoplasm present in more than just a couple of chambers that were connected and included the innermost chambers were chosen. After picking, a drop of concentrated food (pure culture of Nannochloropsis, green colored algae) was added and the foraminifera were left untouched for a night.

Specimens that met one or more of the following criteria were considered as living and used for further procedures:

- Specimens showed a structural infill of cytoplasm with a bright green color, indicating they took up the food over night,

- they developed a film or strings of pseudopodia firmly sticking to sediment particles or food,

- they had covered themselves or gathered a cyst of sediment or food particles. 
Specimens were identified and sorted by species, rinsed, sediment particles were removed, and the color of the cytoplasm was checked before inserting them into a petri dish with calcein $\left(16 \mathrm{mg} \mathrm{l}^{-1}\right)$ (bis[N,Nbis(carboxymethyl)aminomethyl]-fluorescein) (Sigma-Aldrich) for staining (Fig. 2e). Staining took place immediately before the individual culturing phase 0 to 3 and lasted for 14 days. Petri dishes were stored at $8{ }^{\circ} \mathrm{C}$ in a fridge, partial water exchanges and feeding of the foraminifera was performed twice a week. After the staining, the foraminifera were transferred to a petri dish with ASW and left for 1 to 2 days to remove excess calcein from seawater vacuoles in their cytoplasm prior to the introduction into the culturing system.

\subsubsection{Culturing system}

We used two closed-circulation incubation systems for foraminifera (Fig. 2a, b) provided by the Institute of Microbiology, Kiel University (Dagan et al., 2016, their Fig. 5; Woehle et al., 2018, their Fig. S4). The systems were a further development based on earlier closed-circulation systems for foraminiferal culturing (Hintz et al. 2004; Haynert et al., 2011). They were slightly modified for the requirements of this study, but the basic operational principle is described by Dagan et al., 2016. In detail, the systems consisted of three levels with different functions. They are built into Bauknecht WLE 885 fridges for temperature control. Each incubation system accommodated two culturing vessels, which were arranged pairwise on a tray in a polycarbonate cabinet (Fig. 2a, b). The water was pumped from the collection tank at the lowest level of the fridge to the top level into the supply tank. From the supply tank, the water was directed to the culturing vessels and the flow was regulated by 3-way wheels, which ensured that the same amount of water was provided to every culturing vessel. After passing the culturing vessels, the water was redirected to the collection tank. The systems were filled with $15 \mathrm{~L}$ of ASW with a salinity of 30.5 PSU. The water was aerated in the supply and the collection tank with air from outside the system, which passed through a $0.2 \mu \mathrm{m}$ filter. Monitoring of temperature and salinity were performed with a WTW 3210 conductivity meter. Uncertainty of the conductivity measurements was $\pm 0.5 \%$ and $\pm 0.1{ }^{\circ} \mathrm{C}$ for temperature according to the manufacturer's test certificate. All parts that were introduced into the system were sterilized before use either by autoclaving, an UV-lamp exposure, or by applying DanKlorix ${ }^{\circledR}$.

\subsubsection{Preparation for incubation}

For the incubation of the foraminifera, well plates made from PVC were used (Fig. 2c). Each well plate measured 117 x $82 \mathrm{~mm}$ with 104 cavities of which each one had a diameter of $6 \mathrm{~mm}$ and a depth of $5 \mathrm{~mm}$. All well plates had been used in previous experiments for culturing foraminifera in seawater, which ensured that potentially toxic substances or additives were already released from the PVC material (Dagan et al., 2016; Woehle et al., 2018) and should therefore not harm the foraminifera. Before the foraminifera were placed in the cavities, each cavity was filled with sterile quartz sand up to $1.5 \mathrm{~mm}$. The cavities were subsequently filled with artificial seawater and the specimens were inserted randomly. Prepared well plates were left untouched for one night, to make sure that the foraminifera are able to spread their pseudopodial network before incubation. This ensures that they are stable anchored in the cavities and do not float when the culturing vessels are filled and mounted (Haynert et al., 2011). Four well plates respectively were assembled in an airtight Emsa CLIP and CLOSE ${ }^{\circledR}$ box of 226 x 167 x 59 mm (Fig. 2d). Of these four well plates, only three were engrafted with living foraminifera, one species per plate. The fourth plates were left barren and were used to stabilize the arrangement in the culturing vessels. Each culturing 
vessel had a lid with an inflow and an outflow conduit, for which Tygon ${ }^{\circledR}$ tubing were used. The tubing had an outside diameter of $6.3 \mathrm{~mm}$, and the PVC material was considered as nontoxic because no phthalate-based emollients were used. To guarantee that the foraminiferal specimens are not flushed away by the incoming water, the inflow conduit reached almost the bottom of the culturing vessel and was placed between two well plates. The outflow conduit located at the lid of the vessel was attached to a custom-made PVC fitting (Fig. 2d). Once all well plates were arranged in the culturing vessel, the lid was equipped with an additional, elastic sealing and closed. Before the culturing vessels were placed in the incubators, each chamber was slowly filled with ASW. Thereafter, the culturing chambers were placed on the shelve in the incubator, and were connected to the supply hoses. The water supply was turned on once the system was completely filled.

\subsubsection{Culturing experiment}

The culturing experiment had four different phases. The first, phase 0 was dedicated as control phase. No heavy metals were added. This phase allowed both systems to equilibrate in terms of physicochemical and biological processes and made it possible to determine the background values in terms of seawater constituents. This phase lasted 21 days. Afterwards, one system was used as the control system, where no heavy metals were added. In the other system, three phases with elevated heavy metal concentrations were performed. The phases lasted 21 days each. Tropic Marin Pro-Reef salt was mixed with deionized water for adjusting the salinity. A stock solution containing all metals of interest was mixed and before each phase, the heavy metal concentration was elevated by 1 to 1.5 order of magnitude by adding an appropriate amount of the stock solution into the supply tank of the system (see Fig. 2a) (phase $1=1 \mathrm{ml}$, phase $2=10 \mathrm{ml}$, phase $3=150 \mathrm{ml}$ ). The elevation was performed all at once and for keeping the metal concentration at the same level over the culturing phase, a smaller amount of the stock solution was fed into the system bi-weekly. Target concentrations are given in Table 1. The target concentration of the elements at each phase were chosen after earlier culturing experiments with foraminifers $(\mathrm{Mn}, \mathrm{Cu}, \mathrm{Ni}$ : Munsel et al., 2010; Pb: Frontallini et al., 2015 \& 2018a; Zn: Nardelli et al., 2016; Cd: Linshy et al., 2013; Cu: De Nooijer et al., 2007; Le Cadre and Debenary et al., 2006; Cr: Remmelzwaal et al., 2019) to resemble conditions observed in polluted areas like Jakarta Bay (e.g., Williams, 2000) or at European ports (e.g., Fatoki and Mathabatha, 2001). Furthermore, recommended threshold values provided from the EPA (Environmental Protection Agency, USA) were taken into account to ensure that the foraminifera are not limited in their growth and are able to maintain normal physiological functions. However, the heavy metal concentrations in the culturing media obtained during each phase were monitored by frequent water sampling.

Table 1: Heavy metal concentration in the stock solution, target concentration of these metals in each phase and used salt compounds. All salts used were provided in p.a. quality.

\begin{tabular}{lccccc}
\hline & & & \multicolumn{3}{c}{ Target conc. in $\boldsymbol{\mu g ~ l}^{-\mathbf{1}}$} \\
\hline & $\begin{array}{c}\text { Salt } \\
\text { compound }\end{array}$ & $\begin{array}{c}\text { Conc. in mg l-1 } \\
\text { Stock solution }\end{array}$ & $\begin{array}{c}\text { Phase } \\
\mathbf{1}\end{array}$ & $\begin{array}{c}\text { Phase } \\
\mathbf{2}\end{array}$ & $\begin{array}{c}\text { Phase } \\
\mathbf{3}\end{array}$ \\
\hline Chromium $(\mathrm{Cr})$ & $\mathrm{CrCl}_{3} \cdot 6 \mathrm{H}_{2} \mathrm{O}$ & 25 & 0.5 & 5 & 50 \\
Manganese $(\mathrm{Mn})$ & $\mathrm{MnCl}_{2} \cdot 4 \mathrm{H}_{2} \mathrm{O}$ & 40 & 40 & 400 & 4000 \\
Nickel $(\mathrm{Ni})$ & $\mathrm{NiCl}_{2} \cdot 6 \mathrm{H}_{2} \mathrm{O}$ & 5 & 0.1 & 1 & 10 \\
Copper $(\mathrm{Cu})$ & $\mathrm{CuCl}_{2} * 2 \mathrm{H}_{2} \mathrm{O}$ & 2 & 0.05 & 0.5 & 5 \\
Zinc $(\mathrm{Zn})$ & $\mathrm{ZnCl}_{2}$ & 50 & 0.8 & 8 & 80 \\
Cadmium $(\mathrm{Cd})$ & $\mathrm{CdCl}_{2}$ & 4 & 0.08 & 0.8 & 8
\end{tabular}




\begin{tabular}{lccccc} 
Silver $(\mathrm{Ag})$ & $\mathrm{AgNO}_{3}$ & 3.5 & 0.1 & 1 & 10 \\
Tin $(\mathrm{Sn})$ & $\mathrm{SnCl}_{2} * 2 \mathrm{H}_{2} \mathrm{O}$ & 10 & 0.1 & 1 & 10 \\
Mercury $(\mathrm{Hg})$ & $\mathrm{HgCl}_{2}$ & 0.04 & 0.01 & 0.1 & 1 \\
Lead $(\mathrm{Pb})$ & $\mathrm{PbCl}_{2}$ & 10 & 0.1 & 1 & 10 \\
\hline
\end{tabular}

Over the entire culturing period, both systems were exposed to a natural day and night cycle and the flow rate was adjusted to $0.017 \mathrm{ml} \mathrm{s}^{-1}$ (one drop per second) within the culturing vessels. The foraminifera were fed with Nannochloropsis concentrate twice a week $(\sim 2000 \mu \mathrm{g})$. After21 days (meaning after each culturing phase) one culturing vessel per system was exchanged. Vessels and specimens were left in the culturing system for the complete culturing phase (21 days) and no exchange took place during a culturing phase. One culturing vessel containing all three species was left in the system from the beginning until the end of the experiment (from phase 0 to phase 3 ) for 84 days. Data of these specimens are not available due to time constraints caused by the outbreak of the COVID-19 pandemic.

Temperature and salinity were kept stable at $15.0^{\circ} \mathrm{C}$ and 30.2 units (trace metals) and at $14.9^{\circ} \mathrm{C}$ and 30.4 units (control) over the complete culturing period. As the system was mostly closed, evaporation had a minor effect. Demineralized water was added when necessary to keep the salinity stable. The exchanges of culturing vessels between phases inferred a partial water exchange of approximately $10 \%(=1.51)$ every three weeks, which ensured a repetitive renewal of water with adequate quality.

\subsection{Water samples}

\subsubsection{Collection of water samples}

Water samples for determining the heavy metal concentrations were taken frequently from the supply tanks (see Fig. 2a) of both systems using acid cleaned syringes (Norm-Ject ${ }^{\circledR}$ disposable syringe, $20 \mathrm{ml}$, sterile) and sample bottles (LLG narrow neck bottles, $50 \mathrm{ml}$, LDPE = Low Density Polyethylene; Hg: GL 45 Laboratory bottle 250 $\mathrm{ml}$ with blue cap and ring, boro 3.3). Water samples to be analysed for mercury concentrations had to be treated differently due to analytical constraints as detailed below. The water was filtered through a $0.2 \mu \mathrm{m}$ PES filter (CHROMAFIL Xtra disposable filters, membrane material: polyether sulfone pore) for heavy metal samples and through a $0.2 \mu \mathrm{m}$ quartz filter for $\mathrm{Hg}$ samples (HPLC syringe filters, $30 \mathrm{~mm}$ glass fibre syringe filters/ nylon). Filters were rinsed with the sample before the sample was taken. Every water sample was immediately acidified with concentrated ultrapure $\mathrm{HCl}$ to a pH of approximately 2 to avoid changes in the trace metal concentrations due to adsorption to the sample bottle walls or the formation of precipitates.

\subsubsection{Preparation of water samples before analysis}

For $\mathrm{Mn}, \mathrm{Zn}, \mathrm{Ni}, \mathrm{Pb}, \mathrm{Cu}$, and $\mathrm{Cd}$ concentration analyses, the water samples were pre-concentrated offline by using a SeaFAST system (ESI, USA). Twelve $\mathrm{ml}$ of each sample were used to fill a $10 \mathrm{~mL}$ sample loop and concentrated by a factor of 25 into $1.5 \mathrm{M} \mathrm{HNO}_{3}$. All samples were spiked with indium as an internal standard for monitoring and correcting for instrumental drift. Both MilliQ water and bottle blanks of acidified MilliQ water $(\mathrm{pH} \sim 2)$ stored in the same bottles until the samples were passed through the pre-concentration system. Additionally, procedural blanks which were filtered as the samples were also pre-concentrated and measured. A variety of international 
(Open Ocean Seawater NASS-6, River Water SLRS-6, Estuarine Seawater SLEW-3, all distributed by NRCCNRC Canada) and in-house (South Atlantic surface water, South Atlantic Gyre water) reference materials were pre-concentrated like the samples. All samples were subsequently analysed by ICP-MS (inductively coupled plasma mass spectrometry).

For the metals that cannot be preconcentrated by the SeaFAST system as they are not retained on the Nobias resin (Cr, Ag and $\mathrm{Sn}$ ) samples were diluted 1/25 and directly introduced into the ICP-MS. The dilution was performed with indium-spiked nitric acid (2\%) and to match the matrix of these samples blanks and standards with added $\mathrm{NaCl}$ were prepared.

All trace metals except mercury were measured using an Agilent 7500ce quadrupole ICP-MS and raw intensities calibrated with mixed standards, made from single element solutions, covering a wide concentration range. Additionally, a dilution series (dilution factors: 1, 1/10, 1/100 and 1/1000) of SLRS-6 of river water reference material (NRC Canada; Yeghicheyan et al., 2019) was measured for quality control. Mean values and relative standard deviations (RSD) derived from the reference materials are summarised in the appendix (Table A2).

Prior to the measurements of $\mathrm{Hg}$ concentrations, all samples were treated with $\mathrm{BrCl}$ solution at least 24 hours before the analysis to guarantee the oxidation and release of mercury species that are possibly present in a different oxidation states or phases. The $\mathrm{BrCl}$ was removed again from the sample by adding hydroxylamine hydrochloride at least one hour prior to analysis before the $\mathrm{Hg}$ was reduced to the volatile $\mathrm{Hg}^{0}$ species with acidic $\mathrm{SnCl}_{2}(20 \%$ $\mathrm{w} \mathrm{v}^{-1}$ ) during the measuring process. All preparations of the water samples took place in a Clean Lab within a trace metal clean atmosphere and all vials were acid cleaned prior to use. Mercury concentrations were determined using a Total Mercury Manual System (Brooks Rand Model III). The reduced volatile $\mathrm{Hg}^{0}$ is nitrogen-purged onto a gold-coated trap and released from again by heating before it is measured via cold vapour atomic fluorescence (CVAFS) under a continuous argon carrier stream. Quality control of the Hg measurements was carried out by measuring mixed standards, made from single element solutions and confirmed with replicate measurements throughout each analysis. The measurement uncertainty was smaller than $4.5 \%$ RSD for all analyses.

The calcium concentration of culture seawater was analysed using a VARIAN 720-ES ICP-OES (inductively coupled plasma optical emission spectrometer). Yttrium was added as an internal spike and samples were diluted 1/10. IAPSO seawater standard (ORIL) was measured after every 15 samples for further quality control which revealed a measurement uncertainty $<0.35$ (RSD \%) for the elements analysed (mean Ca concentration IAPSO this study $=419.6 \pm 0.15 \mathrm{mg} \mathrm{l}^{-1}$; reference Ca concentration IAPSO Batch $161=423 \mathrm{mg} \mathrm{l}^{-1}$ ).

\subsection{Foraminiferal samples}

After every culturing phase, the culturing vessels were taken out of the system and foraminiferal specimens where collected from their cavities within one day. The individuals were cleaned with tap water and ethanol before they were mounted in cell slides to mechanically remove salt scale and organic coatings with a paintbrush.

In order to check, whether the foraminifera had grown during the experiment, the total number of chambers was counted before and after the experiment for every specimen (Table 2). As the foraminifera were stained with calcein before the experiment, it was possible to cross-check the growth with a fluorescent microscope (Zeiss Axio 
Imager 2) if new chambers without color were added, hence whether the particular specimen had grown or not (Fig. 2e). Only individuals clearly showing new chambers were analysed by Laser ablation ICP - MS.

Prior to the laser ablation analyses, the foraminifera were transferred into individual acid-leached, $500 \mu 1 \mathrm{micro}-$ centrifuge tubes and thoroughly cleaned, applying a procedure adapted from Martin and Lea (2002). The specimens were rinsed three times with MilliQ water and introduced into the ultrasonic bath for a few seconds at the lowest power setting after each rinse. Afterwards, clay and adhering particles were removed by rinsing the sample with Ethanol twice, which was followed by three MilliQ rinses again with minimal ultrasonic treatment. Oxidative cleaning was applied using $250 \mu \mathrm{l}$ of a $0.1 \mathrm{M} \mathrm{NaOH}$ and $0.3 \% \mathrm{H}_{2} \mathrm{O}_{2}$ mixture added to each sample and the vials were kept for $20 \mathrm{~min}$ in a $90{ }^{\circ} \mathrm{C}$ water bath. Afterwards, the samples were rinsed with MilliQ three times to remove the remaining chemicals. The reductive step of the cleaning procedure by Martin and Lea (2002) was not applied. This step is necessary to remove metal oxides, which of course could also influence the trace metal concentration within the foraminiferal shell carbonate but these are usually considered to be added during early deposition (e.g., Boyle, 1983) and therefore unlikely to occur during culture experiments. For Laser Ablation Inductively Coupled Plasma Mass Spectroscopy (LA-ICP-MS) measurements, all cleaned specimens were fixed on a double-sided adhesive tape (PLANO).

Micro-analytical analyses with LA-ICP-MS were performed at the Institute of Geosciences, Kiel University, using a 193nm ArF excimer GeoLasPro HD system (Coherent) with a large volume ablation cell (Zurich-type LDHCLAC, Fricker et al., 2011) and helium as the carrier gas with $14 \mathrm{~mL} \mathrm{~min}^{-1} \mathrm{H}_{2}$ added prior to passing the ablation cell. For the foraminiferal samples, the pulse rate was adjusted to 4 to $5 \mathrm{~Hz}$ with a fluence between 2 and $3.5 \mathrm{~J} \mathrm{~cm}^{-2}$. The spot size was set to 44 or $60 \mu \mathrm{m}$ depending on the size of the foraminiferal chamber. All chambers of a foraminifer that were built up in the culturing medium were analysed, starting from the earliest, inner chamber adjacent to the calcein-stained chamber. The laser was manually stopped once it broke through the foraminiferal shell. The ablated material was analysed by an ICP-MS/MS instrument (8900, Agilent Scientific Instruments) in no gas mode. The NIST SRM 612 glass (Jochum et al., 2011) was used for calibration and monitoring of instrument drift while NIST SRM 614 was measured for quality control. Glasses were ablated with a pulse rate of 10 pulses per second, an energy density of $10 \mathrm{~J} \mathrm{~cm}^{-2}$ and a crater size of $60 \mu \mathrm{m}$. Carbonate matrix reference materials coral JCp-1, giant clam JCt-1, limestone ECRM752-1 and synthetic spiked carbonate MACS-3 (Inoue et al., 2004; Jochum et al., 2019) in the form of nano-particle pellets were analysed (Garbe-Schönberg and Müller, 2014). MACS-3 was used for calibrating the mercury content in the samples as $\mathrm{Hg}$ is not present in the NIST SRM glasses. All results for the reference materials are displayed in the appendix (Table A3). Trace element-to-calcium ratios were quantified using the following isotopes: ${ }^{26} \mathrm{Mg},{ }^{27} \mathrm{Al},{ }^{52} \mathrm{Cr},{ }^{55} \mathrm{Mn},{ }^{60} \mathrm{Ni},{ }^{63} \mathrm{Cu},{ }^{65} \mathrm{Cu},{ }^{68} \mathrm{Zn},{ }^{107} \mathrm{Ag},{ }^{111} \mathrm{Cd}$, ${ }^{114} \mathrm{Cd},{ }^{118} \mathrm{Sn},{ }^{201} \mathrm{Hg},{ }^{202} \mathrm{Hg}$ and ${ }^{208} \mathrm{~Pb}$ normalised to ${ }^{43} \mathrm{Ca}$. If more than one isotope was measured for an element, the average concentration of these was used after data processing. Uncertainty (in \% RSD) was better than $5 \%$ for all TE/Ca ratios. The lowest RSD \% based on the NIST SRM 612 glass was $2.1 \%$ for Mn/Ca and the highest $5.0 \%$ for $\mathrm{Ag} / \mathrm{Ca}$. Uncertainties of all used standards and reference materials are expressed are summarized in Table A3. Each acquisition interval lasted for 90 seconds, started and ended with measuring $20 \mathrm{~s}$ of gas blank, used as the background baseline to subtract from sample intensities during the data reduction process. Furthermore, the background monitoring ensured that the system was flushed properly after a sample. In cases when foraminiferal test walls were very fragile causing the test to break very quickly and, hence, the length of the sample data acquisition interval was less than 15 seconds, these profiles were excluded from further consideration. 
Transient logs of raw intensities given in counts per seconds for all isotopes measured were processed with the software Iolite (Version 4, Paton et al., 2011) producing averages of every time-resolved laser profile. The determination of element/Ca ratios were performed after the method of (Rosenthal et al., 1999). High values of ${ }^{25} \mathrm{Mg},{ }^{27} \mathrm{Al}$ or ${ }^{55} \mathrm{Mn}$ at the beginning of an ablation profile were related to contamination on the surface of the foraminiferal shell or remains of organic matter (e.g., Eggins et al., 2003) and these parts of the profiles were excluded from further data processing. The detection limit was defined by $3.3 * \mathrm{SD}$ of the gas blank in counts per seconds for every element in the raw data. Only values above this limit were used for further analyses. After processing the data with Iolite, an outlier detection of the TE/Ca ratios of the samples was performed. If trace metal values from a spot deviated more than \pm 2 SD from the average of the samples from the corresponding culturing phase, values were defined as outliers and discarded. The number of rejected points is indicated in the supplementary material (Table S1).

All statistical tests were carried out using the statistical program PAST (Hammer, 2001). As the concentration of heavy metals in seawater was varying during individual phases in the metal system (Table A1 and Fig. B1 in the appendix), the mean concentration was calculated by applying an individual curve fit for every phase. The curve was either linear, exponential or a power function depending on the covariance trend the particular metal showed. Based on these curves, water values were calculated for every day and the weighted average from all days was used for further calculations. This ensured that high concentrations in the beginning of each phase did not influence the mean value disproportionately. The partition coefficients of the different trace metal-to-calcium ratios were calculated using the trace element (TE) and calcium ratios in calcite and seawater. The following equation was used:

$$
\mathrm{D}_{\mathrm{TE}}=(\mathrm{TE} / \mathrm{Ca})_{\text {calcite }} /(\mathrm{TE} / \mathrm{Ca})_{\text {seawater }}
$$

When the correlation between the metal concentration in seawater and the metal concentration in the foraminiferal test was positive and significant $\left(\mathrm{R}^{2}>0.4, \mathrm{p}<0.05\right)$, the $\mathrm{D}_{\mathrm{TE}}$ 's are derived from the mean values of all phases and represent the slope of the calculated regression line. In cases where a significant positive correlation between phases could not be identified, the $\mathrm{D}_{\mathrm{TE}}$ values were calculated from the means of each phase separately and the ranges given.

\section{Results}

\subsection{Survival Rates/ Growth rates / Reproductions}

Table 2: Number of inserted and recovered foraminifera from the different systems $(\mathrm{C}=$ control system, $\mathrm{M}=$ metal system) and phases (0-3). Numbers of living individuals after the experiment and individuals that formed chambers during their individual culturing phase are given in $\%$. Note that the percentage of living foraminifera is based on the number of foraminifera that could be recovered alive and not on the number of inserted individuals. The number of laser spots is indicated as well.

\begin{tabular}{lccccccccc}
\hline & C0 & C1 & C2 & C3 & M0 & M1 & M2 & M3 & Total \\
\hline No. of inserted individuals & & & & & & & & & \\
Ammonia aomoriensis & 50 & 24 & 20 & 20 & 19 & 70 & 70 & 72 & 345 \\
Ammonia batava & 22 & 20 & 20 & 20 & 16 & 43 & 72 & 72 & 285
\end{tabular}




\begin{tabular}{lccccccccc} 
Elphidium excavatum & 45 & 24 & 20 & 20 & 19 & 70 & 69 & 70 & 337 \\
Total & 117 & 68 & 60 & 60 & 54 & 183 & 211 & 214 & 967 \\
\hline No. of recovered individuals & & & & & & & & & \\
Ammonia aomoriensis & 43 & 20 & 10 & 19 & 11 & 57 & 58 & 56 & 274 \\
Ammonia batava & 11 & 15 & 16 & 14 & 7 & 29 & 65 & 56 & 213 \\
Elphidium excavatum & 36 & 20 & 20 & 14 & 7 & 62 & 58 & 53 & 270 \\
Total & 90 & 55 & 46 & 47 & 25 & 148 & 181 & 165 & 757 \\
\hline Living individuals (end of experiment) in \% & & & & & & & \\
Ammonia aomoriensis & 86 & 100 & 80 & 100 & 90.9 & 100 & 81 & 98.2 & 92.0 \\
Ammonia batava & 81.8 & 100 & 100 & 92.9 & 100 & 100 & 100 & 100 & 96.8 \\
Elphidium excavatum & 91.7 & 100 & 95 & 92.9 & 100 & 88.7 & 91.4 & 94.3 & 94.3 \\
Total & 86.5 & 100 & 91.7 & 95.3 & 97.0 & 96.2 & 90.8 & 97.5 & 94.4 \\
\hline Ind. that formed chambers (end of the experiment) in \% & & & & & \\
Ammonia aomoriensis & 62.8 & 84.2 & 100 & 93.8 & 81.8 & 100 & 92.3 & 90 & 88.1 \\
Ammonia batava & 45.5 & 85.7 & 100 & 100 & 71.4 & 100 & 100 & 100 & 87.8 \\
Elphidium excavatum & 69.4 & 65 & 56.3 & 38.5 & 57.1 & 67.7 & 75 & 62.3 & 61.4 \\
Total & 59.2 & 78.3 & 85.4 & 77.4 & 70.1 & 89.2 & 89.1 & 84.1 & 79.1 \\
\hline No. of laser spots & & & & & & & & & \\
Ammonia aomoriensis & 22 & 18 & 17 & 20 & 9 & 39 & 40 & 36 & 201 \\
Ammonia batava & 14 & 20 & 19 & 19 & 6 & 17 & 52 & 57 & 204 \\
Elphidium excavatum & 14 & 13 & 13 & 12 & 1 & 36 & 24 & 31 & 144 \\
Total & 50 & 51 & 49 & 51 & 16 & 92 & 116 & 124 & 549 \\
\hline & & & & & & & & \\
\hline
\end{tabular}

On average $74.5 \%$ of the specimens inserted into the experiment could be recovered after their individual culturing phase of 21 days and $94.4 \%$ of these recovered specimens survived. Approximately $79.1 \%$ of the surviving specimens also formed at least one new chamber. Fewer specimens of E. excavatum formed new chambers (61.4 $\%$ ) than A. batava (87.8\%) or A. aomoriensis (88.1\%) (Table 2). On average, E. excavatum formed only one or rarely two new chambers, whereas both Ammonia species formed usually more than four new chambers. Reproduction happened very sporadically concerning between 2 and 6 specimens per phase, on average $5 \%$, for the two Ammonia species but not for E. excavatum. No malformed chambers were observed in specimens that were recovered from the trace-metal contaminated system.

\subsection{Culturing media}

Table 3: Weighted mean TE/Ca values in the culturing medium of the control and the metal system \pm the standard error of the mean (standard deviation $\sigma / \sqrt{ } \mathrm{n}$ ). Furthermore, the factors between the target concentrations (Table 1) and the measured concentrations as well as the factors between individual phases are given. Values given without a standard error originate from only one measurement. All values the calculations are based on can be found in the appendix (Table A1). BDL = below detection limit. 
https://doi.org/10.5194/bg-2021-158

Preprint. Discussion started: 12 August 2021

(c) Author(s) 2021. CC BY 4.0 License.

\begin{tabular}{|c|c|c|c|c|c|c|c|c|c|c|}
\hline $\begin{array}{l}\text { Control } \\
\text { System }\end{array}$ & $\begin{array}{l}\mu \mathrm{mol} \\
\mathrm{mol}^{-1}\end{array}$ & $\begin{array}{l}\mathrm{mmol} \\
\mathrm{mol}^{-1} \\
\end{array}$ & $\begin{array}{l}\mu \mathrm{mol} \\
\mathrm{mol}^{-1}\end{array}$ & $\begin{array}{l}\mu \mathrm{mol} \\
\mathrm{mol}^{-1}\end{array}$ & $\begin{array}{l}\mu \mathrm{mol} \\
\mathrm{mol}^{-1}\end{array}$ & $\begin{array}{l}\mu \mathrm{mol} \\
\mathrm{mol}^{-1}\end{array}$ & $\begin{array}{l}\mu \mathrm{mol} \\
\mathrm{mol}^{-1}\end{array}$ & $\begin{array}{l}\mu \mathrm{mol} \\
\mathrm{mol}^{-1}\end{array}$ & $\begin{array}{c}\mathrm{nmol} \\
\mathrm{mol}^{-1}\end{array}$ & $\begin{array}{l}\mu \mathrm{mol} \\
\mathrm{mol}^{-1}\end{array}$ \\
\hline Phase 0 & BDL & $\begin{array}{c}0.94 \pm \\
0.02\end{array}$ & $\begin{array}{c}7.0 \pm \\
0.1\end{array}$ & $\begin{array}{c}9.3 \pm \\
4.3\end{array}$ & $\begin{array}{c}118.3 \pm \\
4.5\end{array}$ & $\begin{array}{c}0.43 \pm \\
0.214\end{array}$ & $\begin{array}{c}0.41 \pm \\
0.001\end{array}$ & $\begin{array}{c}2.2 \pm \\
0.4\end{array}$ & $\begin{array}{c}5.8 \pm \\
0.6\end{array}$ & $\begin{array}{c}0.44 \pm \\
0.06\end{array}$ \\
\hline Phase 1 & BDL & $\begin{array}{c}0.92 \pm \\
0.00\end{array}$ & $\begin{array}{c}6.3 \pm \\
0.1\end{array}$ & $\begin{array}{c}4.4 \pm \\
1.4\end{array}$ & $\begin{array}{c}91.6 \pm \\
1.1\end{array}$ & $\begin{array}{c}0.19 \pm \\
0.013\end{array}$ & $\begin{array}{c}0.41 \pm \\
0.002\end{array}$ & $\begin{array}{c}2.5 \pm \\
0.1\end{array}$ & $\begin{array}{c}4.5 \pm \\
1.0\end{array}$ & $\begin{array}{c}0.39 \pm \\
0.02\end{array}$ \\
\hline Phase 2 & $\begin{array}{c}1.3 \pm \\
0.3\end{array}$ & $\begin{array}{c}0.90 \pm \\
0.02\end{array}$ & $\begin{array}{c}5.7 \pm \\
0.1\end{array}$ & $\begin{array}{c}2.1 \pm \\
0.2\end{array}$ & $\begin{array}{c}74.8 \pm \\
2.0\end{array}$ & $\begin{array}{l}0.19 \pm \\
0.003\end{array}$ & $\begin{array}{c}0.38 \pm \\
0.006\end{array}$ & $\begin{array}{c}2.1 \pm \\
0.1\end{array}$ & $\begin{array}{c}13.2 \pm \\
5.8\end{array}$ & $\begin{array}{c}0.31 \pm \\
0.02\end{array}$ \\
\hline Phase 3 & $\begin{array}{c}2.0 \pm \\
0.4\end{array}$ & $\begin{array}{c}0.89 \pm \\
0.01 \\
\end{array}$ & $\begin{array}{c}6.8 \pm \\
0.3 \\
\end{array}$ & $\begin{array}{c}1.5 \pm \\
0.1 \\
\end{array}$ & $\begin{array}{c}78.3 \pm \\
0.8\end{array}$ & $\begin{array}{c}0.16 \pm \\
0.009\end{array}$ & $\begin{array}{c}0.37 \pm \\
0.006\end{array}$ & $\begin{array}{c}1.8 \pm \\
0.1 \\
\end{array}$ & $\begin{array}{c}5.8 \pm \\
1.8\end{array}$ & $\begin{array}{c}0.28 \pm \\
0.01 \\
\end{array}$ \\
\hline $\begin{array}{l}\text { Metal } \\
\text { System }\end{array}$ & $\begin{array}{l}\mu \mathrm{mol} \\
\mathrm{mol}^{-1}\end{array}$ & $\begin{array}{l}\mathrm{mmol} \\
\mathrm{mol}^{-1}\end{array}$ & $\begin{array}{l}\mu \mathrm{mol} \\
\mathrm{mol}^{-1}\end{array}$ & $\begin{array}{l}\mu \mathrm{mol}^{-1} \\
\mathrm{~mol}^{-1}\end{array}$ & $\begin{array}{l}\mu \mathrm{mol} \\
\mathrm{mol}^{-1}\end{array}$ & $\begin{array}{l}\mu \mathrm{mol} \\
\mathrm{mol}^{-1}\end{array}$ & $\begin{array}{l}\mu \mathrm{mol} \\
\mathrm{mol}^{-1}\end{array}$ & $\begin{array}{l}\mu \mathrm{mol}^{-1} \\
\mathrm{~mol}^{-1}\end{array}$ & $\begin{array}{l}\mathrm{nmol} \\
\mathrm{mol}^{-1}\end{array}$ & $\begin{array}{l}\mu \mathrm{mol}^{-1} \\
\mathrm{~mol}^{-1}\end{array}$ \\
\hline Phase 0 & $\begin{array}{c}8.0 \pm \\
1.8\end{array}$ & $\begin{array}{c}0.84 \pm \\
0.01\end{array}$ & $\begin{array}{c}7.4 \pm \\
0.1\end{array}$ & $\begin{array}{c}12.9 \pm \\
4.5\end{array}$ & $\begin{array}{c}104.8 \pm \\
1.4\end{array}$ & $\begin{array}{c}0.09 \pm \\
0.02\end{array}$ & $\begin{array}{c}0.43 \pm \\
0.002\end{array}$ & $\begin{array}{c}3.0 \pm \\
0.1\end{array}$ & 5.28 & $\begin{array}{c}0.50 \pm \\
0.04\end{array}$ \\
\hline Phase 1 & $\begin{array}{c}8.6 \pm \\
0.5\end{array}$ & $\begin{array}{c}0.83 \pm \\
0.004\end{array}$ & $\begin{array}{c}7.3 \pm \\
0.1\end{array}$ & $\begin{array}{c}2.8 \pm \\
0.3\end{array}$ & $\begin{array}{c}95.2 \pm \\
0.3\end{array}$ & $\begin{array}{c}0.10 \pm \\
0.02\end{array}$ & $\begin{array}{c}1.12 \pm \\
0.01\end{array}$ & $\begin{array}{c}4.1 \pm \\
0.1\end{array}$ & $\begin{array}{c}39.7 \pm \\
2.7\end{array}$ & $\begin{array}{c}0.69 \pm \\
0.03\end{array}$ \\
\hline Phase 2 & $\begin{array}{c}14.7 \pm \\
0.1\end{array}$ & $\begin{array}{c}0.81 \pm \\
0.003\end{array}$ & $\begin{array}{c}9.6 \pm \\
0.1\end{array}$ & $\begin{array}{c}2.4 \pm \\
0.2\end{array}$ & $\begin{array}{c}134.8 \\
\pm 0.5\end{array}$ & $\begin{array}{c}0.40 \pm \\
0.14\end{array}$ & $\begin{array}{c}4.86 \pm \\
0.03\end{array}$ & $\begin{array}{l}5.2 \pm \\
0.03\end{array}$ & $\begin{array}{c}337.6 \pm \\
52.1\end{array}$ & $\begin{array}{c}2.63 \pm \\
0.3\end{array}$ \\
\hline Phase 3 & $\begin{array}{c}36.3 \pm \\
1.9\end{array}$ & $\begin{array}{c}1.41 \pm \\
0.004\end{array}$ & $\begin{array}{c}61.3 \pm \\
1.8\end{array}$ & $\begin{array}{c}4.0 \pm \\
1.0\end{array}$ & $\begin{array}{c}547.5 \pm \\
20.5\end{array}$ & $\begin{array}{c}6.1 \pm \\
2.5 \\
\end{array}$ & $\begin{array}{c}78.92 \pm \\
1.9 \\
\end{array}$ & $\begin{array}{c}7.5 \pm \\
1.0 \\
\end{array}$ & $\begin{array}{r}3132.4 \\
\pm 323.7 \\
\end{array}$ & $\begin{array}{c}57.84 \pm \\
6.4 \\
\end{array}$ \\
\hline \multicolumn{11}{|c|}{ Factor between target conc. and measured conc. } \\
\hline Phase 1 & 17.2 & 20.8 & 73.0 & 56.0 & 119.0 & 1.0 & 14.0 & 41.0 & 4.0 & 6.9 \\
\hline Phase 2 & 2.9 & 2.0 & 9.6 & 4.8 & 16.9 & 0.4 & 6.1 & 5.2 & 3. & 2.6 \\
\hline Phase 3 & 0.7 & 0.4 & 6.1 & 0.8 & 6.8 & 0.6 & 9.9 & 0.8 & 3.1 & 5.8 \\
\hline \multicolumn{11}{|c|}{ Factor between Phases } \\
\hline Phase $0-1$ & 1.1 & 1.0 & 1.0 & 0.2 & 0.9 & 1.1 & 2.6 & 1.4 & 7.5 & 1.4 \\
\hline Phase 1 & 1.7 & 1.0 & 1.3 & 0.9 & 1.4 & 4.0 & 4.3 & 1.3 & 8.5 & 3.8 \\
\hline Phase 2-3 & 2.5 & 1.7 & 6.4 & 1.7 & 4.1 & 15.3 & 16.2 & 1.4 & 9.3 & 22.0 \\
\hline
\end{tabular}


https://doi.org/10.5194/bg-2021-158

Preprint. Discussion started: 12 August 2021

(c) Author(s) 2021. CC BY 4.0 License.
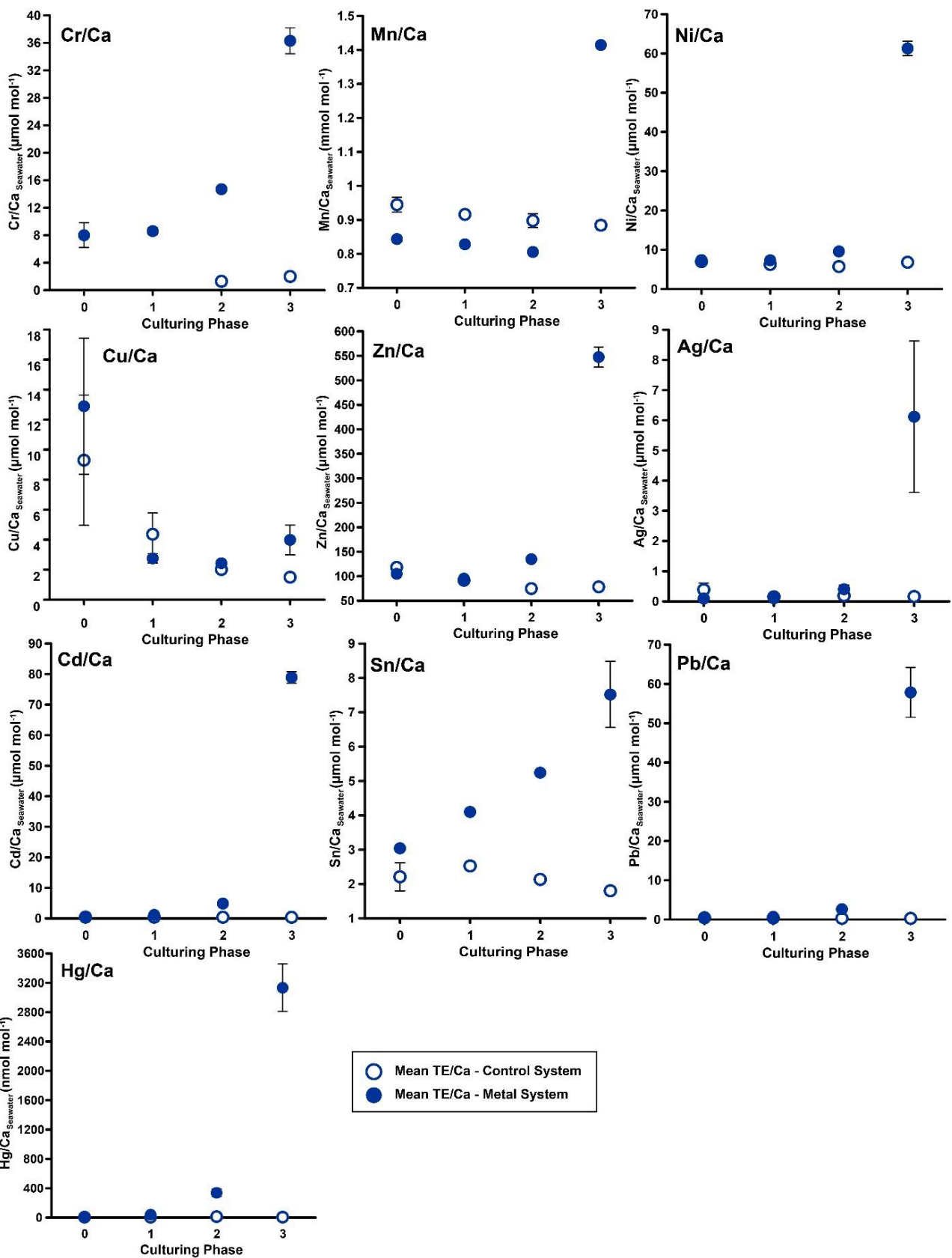

Figure 3: Weighted mean TE/Ca values in the culturing medium in $\mu \mathrm{mol} \mathrm{mol}^{-1}$. Error bars display the standard error of the mean (standard deviation $\sigma / \sqrt{ } \mathrm{n}$ ). Open symbols represent the control system, where no extra metals were added during the complete culturing period (phase 0 to 3 ) and closed symbols represent the metal system. In this system, phase 0 is the control phase without any extra added metals and for phase 1 to 3 , the heavy metal concentration in the culturing medium was elevated. Note that the standard error is comparably high in phase 3 because the heavy metal concentration in this phase varied more strongly, which is shown in the appendix (Table 
A1, Fig. B1). Therefore, this error is derived from the real values in the seawater and not from analytical uncertainties. Note that the $\mathrm{Cr} / \mathrm{Ca}$ values from the control system in phase 0 and 1 are not given as these values were below the detection limit.

In phases 1 and 0 the concentration in both systems were nearly equal for most elements. Only $\mathrm{Cr}$ and $\mathrm{Sn}$ had slightly elevated concentrations in the metal system, whereas $\mathrm{Cu}$ and $\mathrm{Mn}$ concentration were higher in the control system in phase 0 (Fig. 3). This also holds true for Mn in phase 2, when all other metals showed higher concentrations in the metal system than in the control system. In phase 3, the concentration of all heavy metals were elevated in the metal system as compared to the control system. The variation of the metal concentration was in both systems highest in phase 3 for all elements but $\mathrm{Cu}$, which showed the highest variation in phase 0 (Fig. 3). The control system generally displayed a smaller degree of variation than the metal system.

The target concentration of the metals was not accomplished for most metals in phase 1 and 2, the only exception is $\mathrm{Ag}$ in phase 1 (Table 3). The factors between the target and measured concentration was highest (> 50) for $\mathrm{Ni}$, $\mathrm{Cu}$ and $\mathrm{Zn}$ in phase 1 and gets smaller in phase 2 and 3. Generally, all elements but Mn were concentrated higher in phases 1 and 2 than expected. In phase $3 \mathrm{Cr}, \mathrm{Mn}, \mathrm{Cu}, \mathrm{Ag}$ and $\mathrm{Sn}$ reached concentrations closer (factor $0.4-0.8$ ) to the target concentration and $\mathrm{Ni}, \mathrm{Zn}, \mathrm{Cd}, \mathrm{Hg}$ and $\mathrm{Pb}$ were concentrated higher (factor 3.1 - 9.9) than expected. Furthermore, the factor between individual phases (Table 3) was small for the transition from phase 0 to 1 (factor < 1.4) for all elements but $\mathrm{Cd}$ (factor 2.6) and $\mathrm{Hg}$ (factor 7.5). Same patterns can be seen between phase 1 and 2, while the difference between phase 2 and 3 was more distinct (factor > 4) for $\mathrm{Ni}, \mathrm{Zn}, \mathrm{Ag}, \mathrm{Cd}, \mathrm{Pb}$ and $\mathrm{Hg}$. $\mathrm{Mn}, \mathrm{Cu}$ and $\mathrm{Sn}$ showed little variation between phase 2 and 3 (factor < 1.7). Generally, the factor between each phase should have been approximately 10, which was not achieved in most cases. Exceptions were $\mathrm{Ag}, \mathrm{Cd}$ and $\mathrm{Pb}$, which had factors $>15$ between phase 2 and 3. Furthermore, $\mathrm{Hg}$ showed concentrations that were higher by a factor around 10 between all phases (phase $0-1=7.5$, phase $1-2=8.5$, phase $2-3=9.3$ ).

\subsubsection{Incorporation of trace metals into the foraminiferal shell}

Table 4: Mean trace element-to-calcium values of A. aomoriensis, A. batava and E. excavatum in the control and the metal system. Errors are standard errors of the mean (standard deviation $\sigma / \sqrt{ } \mathrm{n}$ ). Values marked with an asterisk were derived from only one laser spot and thus are not considered for further discussion. Furthermore, the calculated $\mathrm{D}_{\mathrm{TE}}$ values, the slope of the linear regression line (OLS - Ordinary Least Squares) of all means, Pearson's correlation coefficient $\mathrm{R}^{2}$ and its significance (p) are given for the calculation with all phases and when removing phase 3 from the calculations. It's also indicated whether the regression line is forced through the origin or not. In cases when a regression did not show significant correlation, the $\mathrm{D}_{\mathrm{TE}}$ range separately calculated from the individual phases is given. In cases when the regression was significant, the $\mathrm{D}_{\mathrm{TE}}$ values represent the slope of the regression line. $\mathrm{Ph}=$ Phase. Values in Table $\mathrm{S} 1$ are the basis of all calculations.

\begin{tabular}{lcccccc}
\hline & Phase & $\mathbf{C r} / \mathbf{C a}$ & $\mathbf{M n} / \mathbf{C a}$ & $\mathbf{N i} / \mathbf{C a}$ & $\mathbf{C u} / \mathbf{C a}$ & $\mathbf{Z n} / \mathbf{C a}$ \\
\hline Control System & & $\mu \mathrm{mol} \mathrm{mol}^{-1}$ & $\mathrm{mmol} \mathrm{mol}^{-1}$ & $\mu \mathrm{mol} \mathrm{mol}^{-1}$ & $\mu \mathrm{mol} \mathrm{mol}^{-1}$ & $\mu \mathrm{mol} \mathrm{mol}^{-1}$ \\
\hline & 0 & $18.6 \pm 2.5$ & $0.11 \pm 0.02$ & $1.3 \pm 0.2$ & $5.6 \pm 0.9$ & $53.2 \pm 8.8$ \\
A. aomoriensis & 1 & $12.6 \pm 0.6$ & $0.53 \pm 0.12$ & $5.9 \pm 0.8$ & $8.6 \pm 1.0$ & $34.2 \pm 4.7$ \\
& 2 & $13.6 \pm 0.5$ & $0.27 \pm 0.07$ & $2.1 \pm 0.2$ & $3.6 \pm 0.2$ & $18.6 \pm 1.9$ \\
& 3 & $10.2 \pm 0.6$ & $0.43 \pm 0.08$ & $4.3 \pm 0.7$ & $8.1 \pm 2.0$ & $29.5 \pm 6.1$
\end{tabular}




\begin{tabular}{|c|c|c|c|c|c|c|}
\hline \multirow{4}{*}{ A. batava } & 0 & $11.6 \pm 0.7$ & $0.04 \pm 0.01$ & $1.4 \pm 0.2$ & $7.2 \pm 1.1$ & $23.9 \pm 4.5$ \\
\hline & 1 & $10.9 \pm 0.5$ & $0.03 \pm 0.00$ & $2.6 \pm 0.3$ & $5.9 \pm 0.6$ & $17.8 \pm 1.3$ \\
\hline & 2 & $9.0 \pm 0.3$ & $0.03 \pm 0.00$ & $0.9 \pm 0.1$ & $5.0 \pm 1.0$ & $12.9 \pm 1.4$ \\
\hline & 3 & $9.1 \pm 0.4$ & $0.03 \pm 0.01$ & $1.9 \pm 0.2$ & $6.5 \pm 1.3$ & $14.9 \pm 2.2$ \\
\hline \multirow{4}{*}{ E. excavatum } & 0 & $22.9 \pm 2.9$ & $0.43 \pm 0.13$ & $9.4 \pm 2.5$ & $22.3 \pm 7.9$ & $28.1 \pm 4.5$ \\
\hline & 1 & $88.9 \pm 34.1$ & $2.29 \pm 0.56$ & $7.8 \pm 1.9$ & $20.3 \pm 8.0$ & $48.9 \pm 12.1$ \\
\hline & 2 & $16.2 \pm 1.7$ & $1.55 \pm 0.26$ & $5.9 \pm 1.0$ & $6.7 \pm 1.4$ & $21.9 \pm 2.9$ \\
\hline & 3 & $26.7 \pm 3.3$ & $1.88 \pm 0.55$ & $4.4 \pm 0.6$ & $4.7 \pm 0.7$ & $16.8 \pm 2.0$ \\
\hline \multicolumn{7}{|l|}{ Metal System } \\
\hline \multirow{4}{*}{ A. aomoriensis } & 0 & $16.0 \pm 0.5$ & $0.08 \pm 0.02$ & $5.5 \pm 0.9$ & $15.2 \pm 2.6$ & $29.8 \pm 5.1$ \\
\hline & 1 & $14.0 \pm 0.7$ & $0.39 \pm 0.08$ & $3.1 \pm 0.3$ & $6.7 \pm 0.7$ & $30.0 \pm 4.0$ \\
\hline & 2 & $11.1 \pm 0.3$ & $0.20 \pm 0.05$ & $5.3 \pm 0.5$ & $5.8 \pm 0.5$ & $28.3 \pm 2.3$ \\
\hline & 3 & $14.1 \pm 1.0$ & $0.71 \pm 0.12$ & $3.8 \pm 0.3$ & $6.3 \pm 1.5$ & $42.2 \pm 6.1$ \\
\hline \multirow{4}{*}{ A. batava } & 0 & $16.5 \pm 0.7$ & $0.07 \pm 0.01$ & $1.1 \pm 0.1$ & $7.7 \pm 1.6$ & $68.0 \pm 9.6$ \\
\hline & 1 & $15.2 \pm 1.2$ & $0.04 \pm 0.01$ & $1.8 \pm 0.3$ & $2.5 \pm 0.6$ & $20.7 \pm 2.7$ \\
\hline & 2 & $9.7 \pm 0.2$ & $0.02 \pm 0.00$ & $1.8 \pm 0.1$ & $8.3 \pm 1.8$ & $12.9 \pm 1.2$ \\
\hline & 3 & $12.2 \pm 0.3$ & $0.17 \pm 0.04$ & $2.9 \pm 0.2$ & $8.3 \pm 1.2$ & $49.8 \pm 3.5$ \\
\hline \multirow{4}{*}{ E. excavatum } & 0 & $17.30^{*}$ & $0.29 *$ & $4.30^{*}$ & $12.20 *$ & $26.70 *$ \\
\hline & 1 & $32.9 \pm 3.4$ & $0.70 \pm 0.12$ & $8.2 \pm 1.1$ & $12.8 \pm 1.8$ & $18.5 \pm 0.9$ \\
\hline & 2 & $41.8 \pm 5.2$ & $0.77 \pm 0.15$ & $8.6 \pm 1.1$ & $11.5 \pm 1.5$ & $29.8 \pm 3.6$ \\
\hline & 3 & $54.1 \pm 8.2$ & $0.88 \pm 0.15$ & $17.0 \pm 2.2$ & $22.6 \pm 3.6$ & $43.1 \pm 3.3$ \\
\hline \multicolumn{7}{|l|}{ Calculations with Phase 3} \\
\hline \multicolumn{7}{|l|}{ A. aomoriensis } \\
\hline Slope of regression line & & & 0.38 & & 1.18 & \\
\hline Correlation coefficient $\left(\mathrm{R}^{2}\right)$ & & & 0.83 & & 0.80 & \\
\hline Significance $(p)$ & & & 0.05 & & 0.05 & \\
\hline DTE & & $0.4-10.3$ & 0.38 & $0.06-0.94$ & 1.18 & $0.08-0.45$ \\
\hline Forced through origin & & $\begin{array}{l}\text { Single } \\
\text { points }\end{array}$ & Yes & $\begin{array}{l}\text { Single } \\
\text { points }\end{array}$ & Yes & $\begin{array}{l}\text { Single } \\
\text { points }\end{array}$ \\
\hline \multicolumn{7}{|l|}{ A. batava } \\
\hline Slope of regression line & & & 0.23 & & & \\
\hline Correlation coefficient $\left(\mathrm{R}^{2}\right)$ & & & 0.84 & & & \\
\hline Significance (p) & & & 0.001 & & & \\
\hline $\mathrm{D}_{\mathrm{TE}}$ & & $0.4-6.8$ & 0.23 & $0.05-0.41$ & $0.60-4.35$ & $0.09-0.65$ \\
\hline Forced through origin & & $\begin{array}{l}\text { Single } \\
\text { points }\end{array}$ & No & $\begin{array}{l}\text { Single } \\
\text { points }\end{array}$ & $\begin{array}{l}\text { Single } \\
\text { points }\end{array}$ & $\begin{array}{l}\text { Single } \\
\text { points }\end{array}$ \\
\hline \multicolumn{7}{|l|}{ E. excavatum } \\
\hline Slope of regression line & & 2.1 & & 0.19 & & \\
\hline Correlation coefficient $\left(\mathrm{R}^{2}\right)$ & & 0.82 & & 0.79 & & \\
\hline Significance $(p)$ & & 0.01 & & 0.003 & & \\
\hline $\mathrm{D}_{\mathrm{TE}}$ & & 2.1 & $0.34-2.50$ & 0.19 & $0.95-5.67$ & $0.08-0.53$ \\
\hline Forced through origin & & Yes & $\begin{array}{l}\text { Single } \\
\text { points }\end{array}$ & Yes & $\begin{array}{l}\text { Single } \\
\text { points }\end{array}$ & $\begin{array}{l}\text { Single } \\
\text { points }\end{array}$ \\
\hline \multicolumn{7}{|l|}{ Calculations without Phase 3} \\
\hline \\
\hline \multirow{2}{*}{\multicolumn{7}{|c|}{$\begin{array}{l}\text { Slope of regression line } \\
\text { Correlation coefficient }\left(\mathrm{R}^{2}\right)\end{array}$}} \\
\hline & & & & & & \\
\hline \multicolumn{7}{|l|}{ Significance $(p)$} \\
\hline $\mathrm{D}_{\mathrm{TE}}$ & & $0.74-10.3$ & $0.09-0.53$ & $0.19-0.94$ & $0.61-5.42$ & $0.21-0.45$ \\
\hline \multirow[t]{2}{*}{ Forced through origin } & & Single & Single & Single & Single & Single \\
\hline & & points & points & points & points & points \\
\hline A. batava & & & & & & \\
\hline Slope of regression line & & & & & & \\
\hline Correlation coefficient $\left(\mathrm{R}^{2}\right)$ & & & & & & \\
\hline Significance $(p)$ & & & & & & \\
\hline $\mathrm{D}_{\mathrm{TE}}$ & & $0.65-6.8$ & $0.02-0.08$ & $0.15-0.41$ & $0.60-4.35$ & $0.10-0.65$ \\
\hline & & Single & Single & Single & Single & Single \\
\hline Forced through origin & & points & points & points & points & points \\
\hline E. excavatum & & & & & & \\
\hline Slope of regression line & & & & & & \\
\hline Correlation coefficient $\left(\mathrm{R}^{2}\right)$ & & & & & & \\
\hline Significance (p) & & & & & & \\
\hline $\mathrm{D}_{\mathrm{TE}}$ & & $2.5-13.4$ & $0.34-2.50$ & $0.64-1.35$ & $0.95-4.73$ & $0.22-0.53$ \\
\hline Forced through origin & & $\begin{array}{l}\text { Single } \\
\text { points }\end{array}$ & $\begin{array}{l}\text { Single } \\
\text { points }\end{array}$ & $\begin{array}{l}\text { Single } \\
\text { points }\end{array}$ & $\begin{array}{l}\text { Single } \\
\text { points }\end{array}$ & $\begin{array}{l}\text { Single } \\
\text { points }\end{array}$ \\
\hline
\end{tabular}


https://doi.org/10.5194/bg-2021-158

Preprint. Discussion started: 12 August 2021

(c) Author(s) 2021. CC BY 4.0 License.

Table 4 continued.

\begin{tabular}{|c|c|c|c|c|c|c|}
\hline & Phase & $\mathrm{Ag} / \mathrm{Ca}$ & $\mathrm{Cd} / \mathrm{Ca}$ & $\mathrm{Sn} / \mathrm{Ca}$ & $\mathrm{Hg} / \mathrm{Ca}$ & $\mathrm{Pb} / \mathrm{Ca}$ \\
\hline Control System & & $\mu \mathrm{mol} \mathrm{mol}^{-1}$ & $\mu \mathrm{mol} \mathrm{mol}^{-1}$ & $\mu \mathrm{mol} \mathrm{mol}{ }^{-1}$ & $\mathrm{nmol} \mathrm{mol}^{-1}$ & $\mu \mathrm{mol} \mathrm{mol}-1$ \\
\hline \multirow[t]{4}{*}{ A. aomoriensis } & 0 & $0.27 \pm 0.08$ & $7.6 \pm 1.0$ & $0.33 \pm 0.07$ & $1.54 \pm 0.46$ & $1.23 \pm 0.22$ \\
\hline & 1 & $0.28 \pm 0.05$ & $3.8 \pm 0.3$ & $1.60 \pm 0.30$ & $3.11 \pm 0.68$ & $1.14 \pm 0.16$ \\
\hline & 2 & $0.16 \pm 0.04$ & $3.6 \pm 0.2$ & $0.21 \pm 0.03$ & $1.13 \pm 0.31$ & $0.81 \pm 0.10$ \\
\hline & 3 & $0.31 \pm 0.11$ & $2.9 \pm 0.2$ & $0.19 \pm 0.03$ & $8.02 \pm 1.72$ & $1.45 \pm 0.42$ \\
\hline \multirow[t]{4}{*}{ A. batava } & 0 & $0.09 \pm 0.03$ & $4.7 \pm 0.5$ & $0.27 \pm 0.05$ & $1.3 \pm 0.4$ & $0.67 \pm 0.10$ \\
\hline & 1 & $0.07 \pm 0.01$ & $2.5 \pm 0.2$ & $0.65 \pm 0.09$ & $1.2 \pm 0.3$ & $0.29 \pm 0.03$ \\
\hline & 2 & $0.05 \pm 0.00$ & $2.7 \pm 0.1$ & $0.08 \pm 0.02$ & $1.5 \pm 0.4$ & $0.39 \pm 0.03$ \\
\hline & 3 & $0.06 \pm 0.01$ & $1.9 \pm 0.1$ & $0.10 \pm 0.02$ & $4.4 \pm 0.6$ & $0.36 \pm 0.05$ \\
\hline \multirow{4}{*}{ E. excavatum } & 0 & $0.22 \pm 0.09$ & $3.6 \pm 1.1$ & $0.99 \pm 0.40$ & $15.0 \pm 4.4$ & $1.83 \pm 0.59$ \\
\hline & 1 & $0.07 \pm 0.01$ & $20.1 \pm 9.2$ & $8.21 \pm 2.63$ & $83.0 \pm 33.4$ & $2.22 \pm 0.54$ \\
\hline & 2 & $0.10 \pm 0.03$ & $1.2 \pm 0.2$ & $0.45 \pm 0.08$ & $16.9 \pm 3.8$ & $0.94 \pm 0.10$ \\
\hline & 3 & $0.04 \pm 0.01$ & $2.3 \pm 0.4$ & $0.27 \pm 0.03$ & $35.8 \pm 6.3$ & $0.55 \pm 0.11$ \\
\hline \multicolumn{7}{|l|}{ Metal System } \\
\hline \multirow[t]{4}{*}{ A. aomoriensis } & 0 & $0.08 \pm 0.03$ & $4.9 \pm 0.3$ & $0.62 \pm 0.09$ & $2.6 \pm 0.6$ & $1.17 \pm 0.24$ \\
\hline & 1 & $0.25 \pm 0.04$ & $4.0 \pm 0.4$ & $0.84 \pm 0.10$ & $1.8 \pm 0.2$ & $0.90 \pm 0.13$ \\
\hline & 2 & $0.52 \pm 0.08$ & $5.5 \pm 0.4$ & $1.70 \pm 0.17$ & $9.1 \pm 1.7$ & $3.85 \pm 0.45$ \\
\hline & 3 & $3.03 \pm 0.39$ & $5.4 \pm 0.4$ & $0.55 \pm 0.10$ & $10.3 \pm 1.3$ & $22.14 \pm 2.37$ \\
\hline \multirow[t]{4}{*}{ A. batava } & 0 & $0.06 \pm 0.03$ & $6.2 \pm 0.2$ & $0.19 \pm 0.04$ & $1.0 \pm 0.2$ & $1.27 \pm 0.08$ \\
\hline & 1 & $0.04 \pm 0.01$ & $3.1 \pm 0.3$ & $0.59 \pm 0.12$ & $0.2 \pm 0.0$ & $0.42 \pm 0.07$ \\
\hline & 2 & $0.18 \pm 0.04$ & $3.1 \pm 0.2$ & $0.46 \pm 0.06$ & $4.5 \pm 1.1$ & $0.52 \pm 0.05$ \\
\hline & 3 & $1.05 \pm 0.17$ & $6.5 \pm 0.3$ & $0.21 \pm 0.02$ & $7.7 \pm 1.0$ & $29.82 \pm 3.70$ \\
\hline \multirow[t]{4}{*}{ E. excavatum } & 0 & $0.40^{*}$ & $5.60 *$ & $0.18^{*}$ & $6.80 *$ & $1.59 *$ \\
\hline & 1 & $0.03 \pm 0.01$ & $3.0 \pm 0.3$ & $2.63 \pm 0.32$ & $85.7 \pm 19.7$ & $1.36 \pm 0.15$ \\
\hline & 2 & $0.69 \pm 0.18$ & $3.9 \pm 0.5$ & $2.89 \pm 0.47$ & $120.4 \pm 44.7$ & $4.61 \pm 0.86$ \\
\hline & 3 & $2.84 \pm 0.64$ & $4.7 \pm 0.5$ & $2.74 \pm 0.42$ & $94.9 \pm 16.2$ & $52.51 \pm 6.17$ \\
\hline \multicolumn{7}{|l|}{ Calculations with Phase 3} \\
\hline \multicolumn{7}{|l|}{ A. aomoriensis } \\
\hline Slope of regression line & & 0.56 & \multirow{5}{*}{$\begin{array}{l}\text { 0.07-18.49 } \\
\text { Single } \\
\text { points }\end{array}$} & \multirow{5}{*}{$\begin{array}{l}0.07-0.63 \\
\text { Single } \\
\text { points }\end{array}$} & \multirow{5}{*}{$\begin{array}{c}0.003-1.39 \\
\text { Single } \\
\text { points }\end{array}$} & 0.39 \\
\hline Correlation coefficient $\left(\mathrm{R}^{2}\right)$ & & 0.97 & & & & 0.97 \\
\hline Significance $(p)$ & & $<0.0001$ & & & & $<0.0001$ \\
\hline $\mathrm{D}_{\mathrm{TE}}$ & & 0.56 & & & & 0.39 \\
\hline Forced through origin & & Yes & & & & Yes \\
\hline \multicolumn{7}{|l|}{ A. batava } \\
\hline Slope of regression line & & 0.17 & \multirow{5}{*}{$\begin{array}{l}\begin{array}{c}0.08-14.42 \\
\text { Single } \\
\text { points }\end{array} \\
\end{array}$} & \multirow{5}{*}{$\begin{array}{l}0.03-0.26 \\
\text { Single } \\
\text { points }\end{array}$} & 0.003 & 0.52 \\
\hline Correlation coefficient $\left(\mathrm{R}^{2}\right)$ & & 0.98 & & & 0.63 & 1 \\
\hline Significance $(p)$ & & $<0.0001$ & & & 0.01 & $<0.0001$ \\
\hline DTE & & 0.17 & & & 0.003 & 0.52 \\
\hline Forced through origin & & Yes & & & Yes & Yes \\
\hline \multicolumn{7}{|l|}{ E. excavatum } \\
\hline Slope of regression line & & 0.47 & & & & 0.91 \\
\hline Correlation coefficient $\left(\mathrm{R}^{2}\right)$ & & 0.96 & & & & 1 \\
\hline Significance $(p)$ & & $<0.0001$ & & & & $<0.0001$ \\
\hline $\mathrm{D}_{\mathrm{TE}}$ & & 0.47 & & $0.06-3.25$ & $0.03-18.51$ & 0.91 \\
\hline Forced through origin & & Yes & $\begin{array}{l}\text { Single } \\
\text { points }\end{array}$ & $\begin{array}{l}\text { Single } \\
\text { points }\end{array}$ & $\begin{array}{l}\text { Single } \\
\text { points }\end{array}$ & Yes \\
\hline Calculations without Phas & & & & & & \\
\hline A. aomoriensis & & & & & & \\
\hline Slope of regression line & & & & & & 1.6 \\
\hline Correlation coefficient $\left(\mathrm{R}^{2}\right)$ & & & & & & 0.91 \\
\hline Significance $(p)$ & & & & & & $<0.001$ \\
\hline DTE & & $0.70-2.57$ & $1.14-18.49$ & $0.10-0.63$ & $0.003-1.39$ & 1.60 \\
\hline Forced through origin & & $\begin{array}{l}\text { Single } \\
\text { points }\end{array}$ & $\begin{array}{l}\text { Single } \\
\text { points }\end{array}$ & $\begin{array}{l}\text { Single } \\
\text { points }\end{array}$ & $\begin{array}{l}\text { Single } \\
\text { points }\end{array}$ & Yes \\
\hline A. batava & & & & & & \\
\hline Slope of regression line & & 0.35 & & & & \\
\hline Correlation coefficient $\left(\mathrm{R}^{2}\right)$ & & 0.91 & & & & \\
\hline Significance $(p)$ & & 0.03 & & & & \\
\hline $\mathrm{D}_{\mathrm{TE}}$ & & 0.35 & $0.63-14.42$ & $0.04-0.26$ & $0.005-0.76$ & $0.20-5.52$ \\
\hline Forced through origin & & Yes & $\begin{array}{l}\text { Single } \\
\text { points }\end{array}$ & $\begin{array}{l}\text { Single } \\
\text { points }\end{array}$ & $\begin{array}{l}\text { Single } \\
\text { points }\end{array}$ & $\begin{array}{l}\text { Single } \\
\text { points }\end{array}$ \\
\hline
\end{tabular}


E. excavatum

Slope of regression line

Correlation coefficient $\left(\mathrm{R}^{2}\right)$

Significance (p)

$\mathrm{D}_{\mathrm{TE}}$

429

Forced through origin

\begin{tabular}{ccccc} 
& & & 0.26 & 2 \\
& & & 0.53 & 0.90 \\
$0.23-4.25$ & $0.80-49.45$ & $0.06-3.25$ & 0.05 & 0.003 \\
Single & Single & Single & No & 2.0 \\
points & points & points & Yes \\
\hline
\end{tabular}

430

Measurable incorporation into the foraminiferal calcite was found for all the trace metals analysed but the degree of incorporation varied profoundly within and between species (Fig. 4 and Table 4). In both systems, the trace metal concentration in E. excavatum was higher than in the other species (A. aomoriensis and A. batava) for Cr, $\mathrm{Mn}, \mathrm{Ni}, \mathrm{Cu}, \mathrm{Hg}$ and $\mathrm{Sn}$. This trend is also visible but less pronounced in the $\mathrm{Cu}$ values of the control system.

$\mathrm{Cr}, \mathrm{Ni}, \mathrm{Cu}, \mathrm{Zn}, \mathrm{Cd}, \mathrm{Pb}$ and $\mathrm{Ag}$ values of A. aomoriensis displayed the highest standard error of the mean paired with highest concentrations in the water in the metal system. Sn, Mn and $\mathrm{Hg}$ did not show any clear pattern. In the control system, all trace metal concentrations had higher standard errors of the mean when the concentration of these metals in the culturing medium was higher. The trend is also shown in A. batava and E. excavatum for all trace metals of the control and the metal system.

Calculations were performed with and without phase 3 (Fig. 4, Fig. B2 and Table 4) to address a possible overload effect when it comes to higher metal concentrations in the seawater.

When phase 3 was included, a strong positive correlation $\left(\mathrm{R}^{2}>0.9, \mathrm{p} \leq 0.05\right)$ of $\mathrm{Ag}$ and $\mathrm{Pb}$ concentrations in the foraminiferal shell and in the culturing medium was recognised in all three species. Furthermore, A. batava also revealed a positive correlation for $\mathrm{Hg}\left(\mathrm{R}^{2}=0.63, \mathrm{p}<0.01\right)$, A. aomoriensis for $\mathrm{Cu}\left(\mathrm{R}^{2}=0.80, \mathrm{p}<0.05\right)$ and $E$. excavatum for $\mathrm{Cr}\left(\mathrm{R}^{2}=0.82, \mathrm{p}<0.01\right)$ and $\mathrm{Ni}\left(\mathrm{R}^{2}=0.79, \mathrm{p}<0.003\right)$. Weaker but still significant positive correlations were recorded for $\mathrm{Mn}\left(\mathrm{R}^{2}>0.84, \mathrm{p} \leq 0.05\right)$ for both Ammonia species. An indistinct correlation of the concentration in the seawater and in the foraminiferal test was recognised for $\mathrm{Zn}$ in all three species, whereas $\mathrm{Cd}$ and Sn showed no covariance (Fig. 4 and Table 4).

When phase 3 was excluded from the calculations, A. aomoriensis and E. excavatum showed a positive correlation for $\mathrm{Pb}\left(\mathrm{R}^{2}>0.9, \mathrm{p} \leq 0.003\right)$, A. batava for $\mathrm{Ag}\left(\mathrm{R}^{2}=0.91, \mathrm{p}=0.03\right)$ and in E. excavatum $\mathrm{Hg}$ correlated weaker positively $\left(R^{2}>0.53, p \leq 0.05\right)$. All other elements show no significant correlation (Fig. 4 and Table 4$)$. 
https://doi.org/10.5194/bg-2021-158

Preprint. Discussion started: 12 August 2021

(c) Author(s) 2021. CC BY 4.0 License.
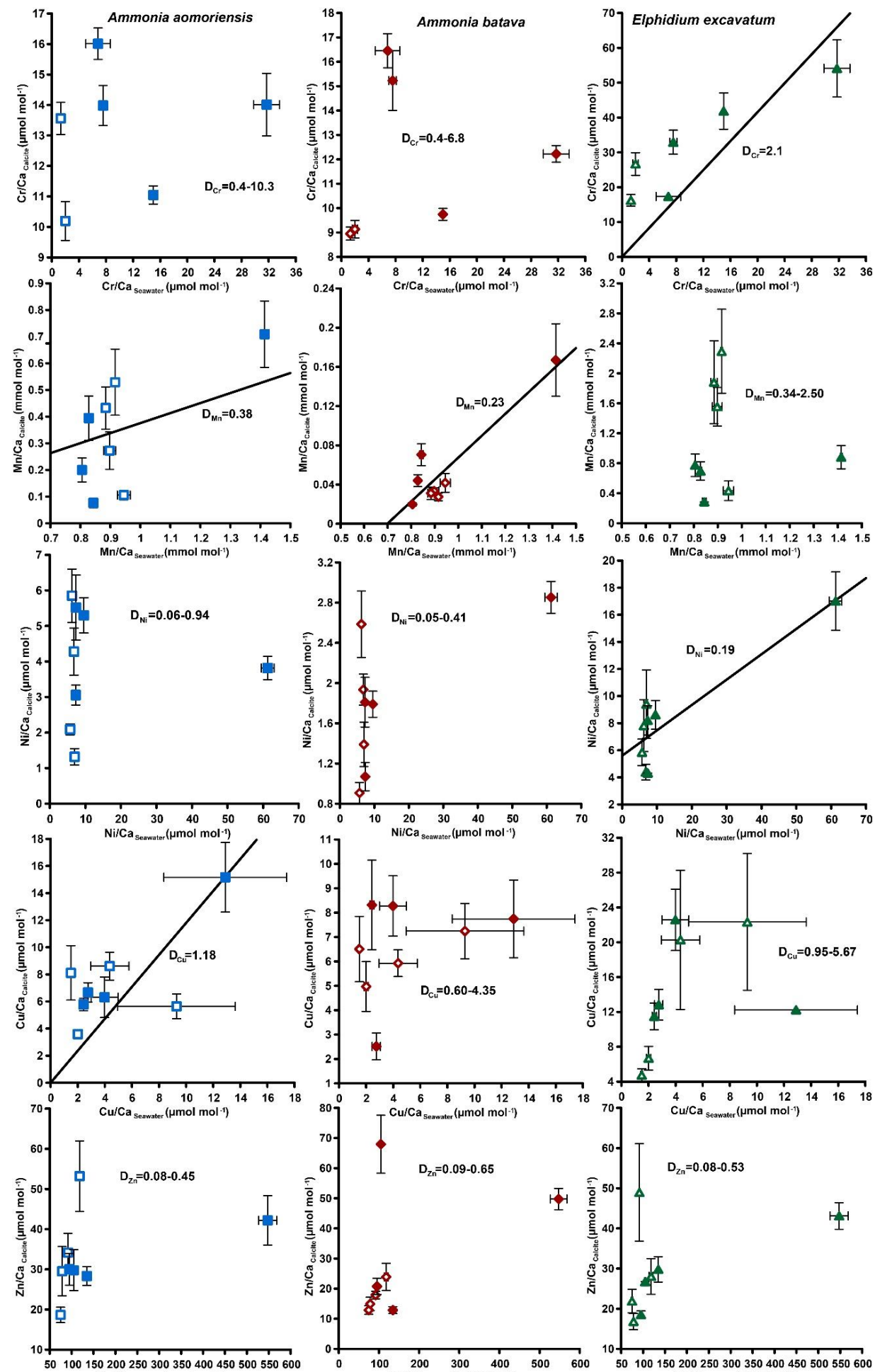
https://doi.org/10.5194/bg-2021-158

Preprint. Discussion started: 12 August 2021

(c) Author(s) 2021. CC BY 4.0 License.
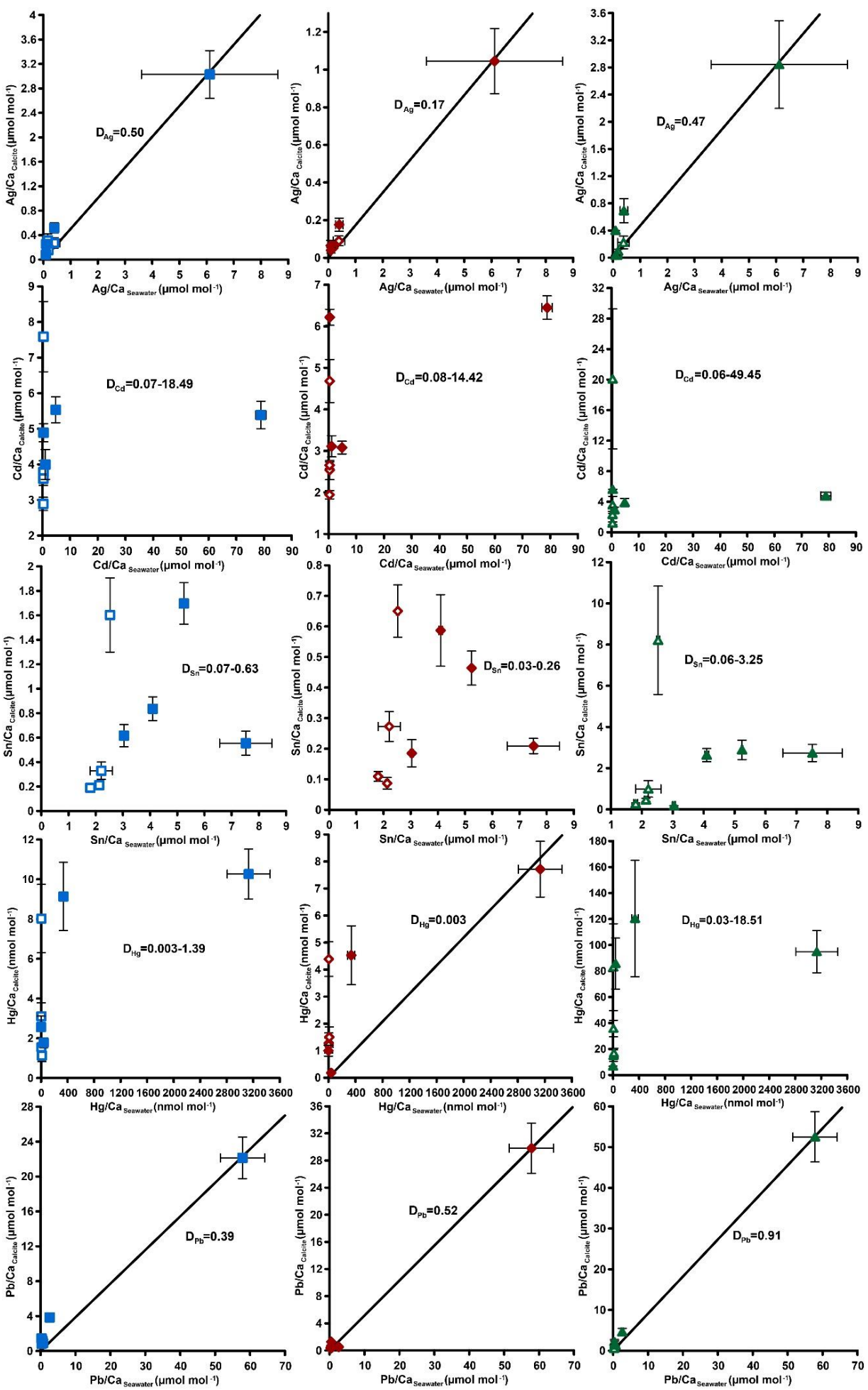


\begin{tabular}{|l|l|}
\hline$\square$ & Mean TE/Ca Control System - A. aomoriensis \\
Mean TE/Ca Metal System - A. aomoriensis \\
Mean TE/Ca Control System - A. batava \\
Mean TE/Ca Metal System - A. batava \\
$\quad \begin{array}{l}\text { Mean TE/Ca Control System - E. excavatum } \\
\text { Mean TE/Ca Metal System - E. excavatum } \\
\text { Regression line }\end{array}$ \\
\hline
\end{tabular}

Figure 4: Mean TE/Ca values in the foraminiferal calcite versus the mean TE/Ca values in the corresponding culturing medium based on phase 0 to 3 . Each data point represents the mean value of all laser ablation ICP - MS measurements on single foraminiferal chambers built up during the individual culturing phase plotted against the mean metal concentrations in the seawater averaged over the culturing phase (Table 3). Error bars symbolize the standard error of the mean. The linear regression line is displayed when elements showed a significant correlation between seawater and calcite. $\mathrm{D}_{\mathrm{TE}}$ 's of E. excavatum where considered without values for Phase 0 of the Metal System as only data from one newly formed chamber are available. All values can be found in Table 4.An enlarged graph based on the calculations without phase 3 is provided in the appendix (Fig. B2).

Partition coefficients for the different trace elements were deduced from molar foraminiferal test TE/Ca and the values from the corresponding culturing medium. Note that the $\mathrm{D}_{\mathrm{TE}}$ values represent the slope of the regression line when there was a positive correlation detected and were furthermore separately calculated for every individual phase. The range of the calculations based on every individual phase are given when no positive correlation could be detected. Furthermore, calculations were performed with and without phase 3 (Fig. 4 , Fig. B2 and Table 4).

Generally, the values varied between 0.003 and 49.45 . The majority of $\mathrm{D}_{\mathrm{TE}}$ were lower than 1 in A. aomoriensis (with phase $3=61 \%$, without phase $3=57 \%$ ) and A. batava (with phase $3=75 \%$, without phase $3=73 \%$ ), i.e., uptake but no enrichment took place. $\mathrm{D}_{\mathrm{TE}}$ values derived from E. excavatum on the other hand showed a smaller proportion $<1$ (with phase $3=47 \%$, without phase $3=42 \%$ ). Note that $\mathrm{D}_{\mathrm{TE}}$ of $E$. excavatum were considered without values for Phase 0 as there were data from only one newly formed chamber available. For most elements $\left(\mathrm{Cr}, \mathrm{Mn}, \mathrm{Ni}, \mathrm{Cu}, \mathrm{Cd}, \mathrm{Sn}, \mathrm{Pb}\right.$ and $\mathrm{Hg}$ ) $\mathrm{D}_{\mathrm{TE}}$ derived from E. excavatum are higher than $\mathrm{D}_{\mathrm{TE}}$ from the two Ammonia species (Table 4, Fig. 4), which showed comparable $\mathrm{D}_{\mathrm{TE}}$ values for most elements. One exception is $\mathrm{Zn}$, where all $\mathrm{D}_{\mathrm{Zn}}$ values are within a similar range $\left(\mathrm{D}_{\mathrm{Zn}} \sim 0.08-0.65\right)$ independent of the species. For $A$. aomoriensis $\mathrm{D}_{\mathrm{Cu}}$ was $>$ 1 and $\mathrm{D}_{\mathrm{Cd}}$ as well as $\mathrm{D}_{\mathrm{Pb}}$ were also $>1$ when phase 3 was excluded from the calculations. A. batava showed $\mathrm{D}_{\mathrm{TE}}$ values > 1 for most $\mathrm{Cr}$ and $\mathrm{Cd}$ values (excluding phase 3 and 2) when considering calculations from the individual phases. E. excavatum displayed $\mathrm{D}_{\mathrm{TE}}$ values $>1$ for $\mathrm{Cr}$ and $\mathrm{Cu}$ for the calculations with phase 3 and also for $\mathrm{Pb}$ without phase 3. Furthermore, $\mathrm{D}_{\mathrm{Cd}}$ and $\mathrm{D}_{\mathrm{Hg}}$ of E. excavatum were also mostly $>1$ when looking at calculations based on individual phases (Table 4, Fig. 4). The highest variation between minimum and maximum $\mathrm{D}_{\mathrm{TE}}$ for all species was found for $\mathrm{Cd}$ and $\mathrm{Hg}$. In addition, some elements showed partition coefficients $>1$ in a couple of phases and/ or in one of the two culturing systems (e.g., E. excavatum: Mn = Control System Phase 1 to 3; $A$. batava: $\mathrm{Cu}=$ all but Control System Phase 0 and Metal System Phase 1; $\mathrm{Pb}=$ Control System and Metal System Phase 1; A. aomoriensis: $\mathrm{Cr}=\mathrm{Control} \mathrm{System} \mathrm{Phase} 2$ and 3, Metal System Phase 1; Ag = Control System Phase 1 and 3, Metal System Phase 2 and 3, Hg: Control System Phase 3, see Table 4, Fig. 4). 


\section{Discussion}

\subsection{Experimental Uncertainties}

The element concentrations within the culturing medium of each culturing phase were comparably stable for most elements in the control system. In the metal system, the variations were higher, which is due to the punctual input of the stock solution for reaching the next phase concentration (Table A1, Fig. B1). This sudden adding of metals resulted in a high peak concentration in the beginning of the new phase, which equilibrated after a while. This trend is most pronounced in phase 3 as the added amount of the stock solution was highest for this phase, which is why the standard error of this phase is comparably high. Furthermore, the variations of the metal concentrations were in a comparable range than those presented in other culturing studies (e.g., Marechal-Abram et al., 2004; De Nooijer et al., 2007; Munsel et al., 2010; Remmelzwaal et al., 2019).

When taking into account that the amount of the stock solution added to the culturing medium of the metal system at the beginning of each culturing phase was elevated 1 to 1.5 order of magnitudes between phases, the measured metal concentrations are smaller than expected for phases 0,1 and 2 . This in combination with the varying metal concentration within one phase suggests that several processes are affecting the concentration in such a complex culturing system. One possible mechanism is sorption of the metals onto surfaces (e.g., tubing, culturing vessels, plates, organic matter or the foraminiferal test itself), which could have lowered the metal concentration in the culturing medium. Therefore, sorption could have contributed to the overall budget of the metals. On the other hand, $\mathrm{Cu}$ appears to have been released from components of the culturing system even though the system was cleaned before and was operated with seawater for 14 days before the experiments begun. For instance, the concentration of $\mathrm{Cu}$ was high in phase 0 , where no metals were added suggesting release from system parts. In phase 1 , the $\mathrm{Cu}$ concentration decreased meaning the contamination derived from the system was removed by a process similar to that observed for the other metals after additions were made. Similar effects have been reported by De Nooijer et al. (2007) for $\mathrm{Cu}$ and Havach et al. (2001) for Cd. Other processes like the uptake of the metals by the foraminifera itself and the growth of algae could further have an influence on the metal concentration in the culturing medium. Such processes are difficult to predict and even more challenging to avoid but probably mirror real environments better than sterile petri dish experiments do (e.g., Havach et al., 2001; Hintz et al., 2004; Munsel et al., 2010).

Neither the survival rate nor the formation of new chambers was influenced by the elevated metal concentrations during the culturing period. These features were rather constant between the four different phases. Furthermore, no malformations of the test morphology were recognised. Elevated heavy metal concentrations are thought to induce a higher rate of malformations in benthic foraminifera (e.g., Sharifi et al., 1991; Yanko et al., 1998), whereas recent studies constrained them as a reaction to stressful environments, not necessarily created by high heavy metal concentrations (Frontalini and Coccioni, 2008; Polovodova and Schönfeld, 2008). The lack of malformations in our experiments suggests that the foraminifera were neither poisoned by elevated trace metal concentrations nor stressed too much by strongly varying environmental parameters, maintaining a normal metabolism and growth. Reproduction was generally very rare, which may indicate that the conditions were not ideal. In field studies foraminiferal reproduction has been linked to short periods of elevated food supply (e.g., Lee et al., 1969; Gooday, 1988; Schönfeld and Numberger, 2007). The regular feeding of foraminifera in our experiment twice a week at constant rates therefore probably did not provide supply levels that trigger reproduction. 
Calcein was used for staining the foraminiferal test before they were placed into the culturing system. Calcein binds to $\mathrm{Ca}$ and is incorporated into the mineralised calcium carbonate (Bernhard et al., 2004). It is conceivable that the trace metal incorporation could also be affected by calcein. However, no evidence has been found by a variety of studies (e.g., Hintz et al., 2006; De Nooijer et al., 2007; Dissard et al., 2009). Furthermore, calcein was only used prior to the experiment to mark the last chamber that was grown outside the culturing system. Therefore, the incorporation of the metals measured in subsequent chambers was not affected by the calcein application.

\subsection{Incorporation of heavy metals in the foraminiferal test}

Many heavy metals have been demonstrated to be incorporated into the foraminiferal shell (e.g., Cr: Remmelzwaal et al., 2019; Mn: Koho et al, 2015; 2017; Cu: De Nooijer et al., 2007; Ni: Munsel et al., 2010; Hg: Frontalini et al., 2018; Cd: Havach et al., 2001; Pb: Titelboim et al., 2018; Sagar et al., 2021; Zn: Marchitto et al., 2000; Van Dijk et al., 2017), and the incorporation of all of these metals was measured here. Additionally, to our knowledge, $\mathrm{Sn}$ and $\mathrm{Ag}$ were investigated here for the first time. The levels observed were well above control values indicating an elevated incorporation of $\mathrm{Ag}$ and $\mathrm{Sn}$ into the foraminiferal test calcite with increasing metal concentrations in seawater.

Different factors can influence the incorporation of these metals into the foraminiferal test. First of all, the uptake depends on metabolical pathways during the calcification process. Fundamental biomineralization processes of foraminifera are the subject of an ongoing discussion and several (partly) competing models have been proposed so far (e.g., Elderfield and Erez, 1996; Erez, 2003; De Nooijer et al., 2009b, 2014; Nehrke et al., 2013). One model proposes that the foraminifera take up ions directly from the surrounding seawater by endocytosis or by building seawater vacuoles, which are transported to the site of calcification (SOC) (Elderfield and Erez, 1996; Erez 2003; De Nooijer et al., 2009b; 2009a; Khalifa et al., 2016). The SOC is located outside the foraminiferal cell and the formation of new calcite takes place in this zone (see e.g., De Nooijer et al., 2014 for a summary and illustration). There are evidence that this part is separated from the surrounding seawater (e.g., Spindler, 1978; Bé et al., 1979; De Nooijer et al., 2009b; 2014; Glas et al., 2012; Nehrke et al., 2013). The other competing model suggests that the uptake of ions and the transport to the SOC is performed directly from the seawater across the cell membrane by active trans-membrane-transports (TMT) and/ or passive transport via gaps in the pseudopodial network of the foraminifera (Nehrke et al., 2013; De Nooijer et al., 2014). The dependency of heavy metal concentrations in the foraminiferal test on their seawater concentration relies on the prevailing mechanism. A biomineralization based on endocytosis does not control the amount of ions that is introduced into the foraminiferal cell and is transported to the SOC. Consequently, processes like Rayleigh fractionation are most important (e.g., Elderfield and Erez, 1996). This would also mean that the metal concentration in the seawater is directly mirrored by their concentration in the foraminiferal shell, which cannot be supported by the results of our study. Indeed, several elements show partition coefficients $>1$ when the $\mathrm{D}_{\mathrm{TE}}$ 's are calculated separately for each phase (see Results section in this manuscript). Only $\mathrm{Pb}$ and $\mathrm{Cr}$ in E. excavatum and $\mathrm{Cu}$ and $\mathrm{Pb}$ in A. aomoriensis consistently display mean $\mathrm{D}_{\mathrm{TE}}{ }^{\prime} \mathrm{s}>$ 1 paired with a positive correlation of the concentration in seawater and in the foraminiferal shell, which could indicate a non-selective uptake of these metals. On the other hand, the $\mathrm{D}_{\mathrm{TE}}$ values of many elements $(\mathrm{Ni}, \mathrm{Zn}, \mathrm{Cd}$, $\mathrm{Hg}, \mathrm{Pb}$ ) dramatically decrease with increasing concentration in the seawater in the highest metal treatment in all species. This kind of overload effect was also noted by Nardelli et al. (2016) for Zn or by Munsel et al. (2010) for Ni. Nardelli et al. (2016) suggested, that biological mechanism expulse or block these metals if the concentration 
is getting too high and an imminent intoxication is probable, which may be managed by controlling the ion uptake via TMT. Therefore, it may well be possible that the highest concentration of the metals in our study was close to the tipping point of the biological mechanism taking over and protecting the organism.

Besides biologically controlled factors, also physicochemical properties play an important role when it comes to the uptake of ions. One chemical factor is the aqueous speciation and solubility of the metals. Metals with a free ion form with a charge of $2+$ are more similar to $\mathrm{Ca}^{2+}$, which makes incorporation more likely (Railsback, 1999). Nearly all metals in this study were added as dissolved chlorides and therefore had a charge of $2+$. The only exceptions were $\mathrm{Ag}$, which was added as $\mathrm{AgNO}_{3}$ with a charge of $1+$ and $\mathrm{Cr}$, which was added as $\mathrm{CrCl}_{3} * 6 \mathrm{H}_{2} 0$. The charge of the cation as such does not seem to make a major difference as Ag was incorporated into all three species and Cr into E. excavatum with a significant positive correlation to concentrations in the culturing medium. Furthermore, it is possible that the oxidative state of the elements is changing due to their $\mathrm{pH}$ dependency, which will be discussed for every element separately. Furthermore, other ions with a charge of 1+ are also known to be incorporated in calcite. Examples are $\mathrm{Li}^{+}$(e.g., Delaney et al., 1985; Hall et al., 2004) and $\mathrm{Na}^{+}$(e.g., Wit et al., 2013; Bertlich et al., 2018), which are believed to occupy interstitial positions in calcite where the calcite lattice has defects (Ishikawa and Ichikuni, 1984; Okumura and Kitano, 1986). In addition, rare earth elements with a charge of $3+$ are also detected in the foraminiferal calcite (e.g., Haley et al., 2005; Roberts et al., 2012).

The aqueous speciation of many metals is strongly influenced by the pH (e.g., Förstner, 1993; Pagnanelli et al., 2003; Spurgeon et al., 2006; Powell et al., 2015; Huang et al., 2017). As the pH during the experiment was stable around $8.0 \pm 0.1$ (measured twice a week), speciation changes between phases due to varying $\mathrm{pH}$ values can be excluded. However, it is possible that some metals were not available in a form that could be readily incorporated in the calcite such as the free ion or carbonate species. $\mathrm{Cr}$ is not available in an optimal speciation to substitute $\mathrm{Ca}$ as a pH of 8 would favour $\mathrm{Cr}^{3+}$ or $\mathrm{Cr}^{4+}$ as well as oxides and hydroxides (Elderfield, 1970; Geisler and Schmidt, 1991). Furthermore, the used Cr-salt may not have dissolved completely, even though the stock solution was heated and stirred during the process. Both in combination may lead to the small variation between the different phases in the seawater. Interferences that could possible influence the $\mathrm{Cr}$ measurements in the water samples are chlorine oxides or hydroxides (e.g., Tan and Horlick, 1986; McLaren et al., 1987, Reed et al., 1994; Laborda et al., 1994). As $\mathrm{NaCl}$ blanks were measured, these interferences are most likely monitored and can be excluded as a biasing factor. Furthermore, measurements of reference materials revealed accurate Cr concentrations compared to those presented in the literature (Table A2), which also corroborates the assumption that these interferences can be neglected. Similar $\mathrm{pH}$ processes could also have effected $\mathrm{Cu}$ as a pH around 8 , like in this experiments, favours copper carbonates over free $\mathrm{Cu}^{2+}$ ions (e.g., Escudero et al., 2008, Millero et al., 2009), which means that the best available speciation was not prevailing during the experiments. Nevertheless, $\mathrm{Cu}$ and $\mathrm{Cr}$ were taken up by all species and therefore, this factor cannot be decisive when it comes to incorporation of these metals into the foraminiferal shell.

If the incorporation of metals would be straightforward and would only depend on the speciation of the metal and other physicochemical factors, the behaviour of the metals would mostly be influenced by the ionic radius in combination with the charge of the metal ions as described for carbonate minerals by Rimstidt et al. (1998). The endocytotic pathway of seawater components into the foraminifer provokes a behaviour of ion incorporation comparable to inorganic calcite precipitation. It was found that cations are incorporated into inorganic calcite by 
substitution of $\mathrm{Ca}^{2+}$ (e.g., Reeder et al., 1999), especially when the effective ionic radius of these ions is comparable to the one of calcium $(=1.0 \AA)$.
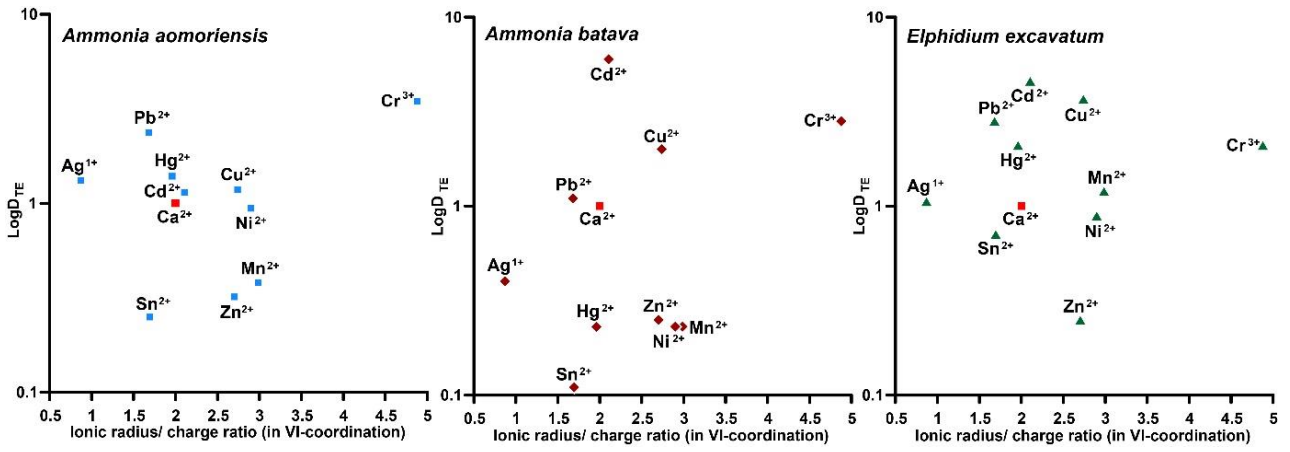

Figure 5: Partition coefficients $\left(\log \mathrm{D}_{\mathrm{TE}}\right.$ ) in dependency of the ionic radius-to-charge ratio (values from Shannon and Prewitt, 1969) in 6-fold coordination for the trace elements analysed in this study. Displayed mean $\mathrm{D}_{\mathrm{TE}}{ }^{\mathrm{S}} \mathrm{s}$ are derived from single values calculated for each culturing phase individually (Metal and Control System Phase 0 to 3 ) and not from the regression lines. Calcium is marked in red. Blue squares represent values from A. aomoriensis (left), red diamonds represent values from A. batava (middle) and green triangles represent values from $E$. excavatum (right).

Some metals like $\mathrm{Mn}, \mathrm{Zn}$ and $\mathrm{Cu}$ are known to be fundamentally necessary as micro-nutrients to maintain biological and physiological function of a cell (e.g., Mertz, 1981; Tchounwou et al., 2012; Martinez-Colon et al., 2009; Maret, 2016). Therefore, these elements should preferentially be taken up into the foraminiferal cell, where they were used for further processes. This in turn could lead to the consumption of these metals before they can be incorporated into the foraminiferal tests. All of these ions have a similar ionic radius $(\mathrm{Cu}=0.73 \AA$, $\mathrm{Mn}=0.67$ $\AA, \mathrm{Zn}=0.74 \AA$ ) in six-fold coordination (Rimstidt et al., 1998), which would also suggest, that their behaviour is comparable. The ionic radii are much smaller than that of $\mathrm{Ca}$, but are rather similar to $\mathrm{Mg}(0.72 \AA$, Rimstidt et al., 1998) (Fig. 5).

Mn shows a positive correlation between its concentration in seawater and the foraminiferal test in the two Ammonia species when the calculations include phase 3. This indicates that this element serves as a well-behaved proxy influenced mainly by its concentration in seawater. However, E. excavatum do not show this positive correlation. Furthermore, $\mathrm{D}_{\mathrm{Mn}}$ values of this study calculated with phase 3 (A. aomoriensis $\mathrm{D}_{\mathrm{Mn}}=0.38$, A. batava $\mathrm{D}_{\mathrm{Mn}}=0.23$, E. excavatum $\left.\mathrm{D}_{\mathrm{Mn}}=0.34-2.50\right)$ are comparable in range of those presented in Koho et al. (2015, 2017: $\left.\mathrm{D}_{\mathrm{Mn}}=0.34-2.50\right)$ and Barras et al. (2018: $\left.\mathrm{D}_{\mathrm{Mn}}=0.09-0.35\right)$, but lower than Munsel et al. (2010) reported (D $>$ 2.4) except for E. excavatum. Species-specific differences in partition coefficients not only for Mn but also for other elements like Mg or Na are common phenomena (e.g., Toyofuku et al., 2011; Barras et al., 2018; Wit et al., 2013) and could also explain the offset of the $\mathrm{D}_{\mathrm{TE}}$ values from E. excavatum in this study. Furthermore, it is known that the presence of toxic metals such as $\mathrm{Cd}$, $\mathrm{Ni}$ or $\mathrm{Hg}$ can inhibit the uptake of essential metals like Mn if these metals are present in low concentrations (e.g., Sunda and Huntsman, 1998a, 1998b). It is possible that this mechanism is more pronounced in E. excavatum than in the Ammonia species. Zn was clearly incorporated above control level into all three species, but it's behaviour is influenced by more factors than the concentration of Zn in 
the culturing medium. This can be inferred by the fact that there was no significant correlation recognised between Zn concentration in calcite and seawater (Fig. 4, Table 4). $\mathrm{D}_{\mathrm{Zn}}$ values of this study are in good agreement with those calculated by Van Dijk et al. (2017) for four hyaline species $\left(\mathrm{D}_{\mathrm{Zn}}=0.2-0.36\right)$ and Nardelli et al. (2016) for the miliolid Pseudotriloculina rotunda $\left(\mathrm{D}_{\mathrm{Zn}}=0.2-4.0\right)$, especially when phase 3 is excluded from the calculation (this study, without phase 3: A. aomoriensis $\mathrm{D}_{\mathrm{Zn}}=0.21-0.45$, A. batava $\mathrm{D}_{\mathrm{Zn}}=0.10-0.65$, E. excavatum $\mathrm{D}_{\mathrm{Zn}}=0.22-$ 0.53). Other studies reported higher values between 3.5 and 9, though for rotaliid taxa like Cibicidoides wullerstorfi and Uvigerina spp. (Marchitto et al., 2000; Van Dijk et al., 2017). It is again possible that the mixture of metals is inhibiting the uptake of essential metals like $\mathrm{Zn}$ similar to $\mathrm{Mn} . \mathrm{Cu}$ shows a simple well-behaved proxy behaviour with a significant positive correlation in A. aomoriensis but not in the other two species. The $\mathrm{D}_{\mathrm{Cu}}$ presented by Munsel et al. (2010) ( $\left.\mathrm{D}_{\mathrm{Cu}}=0.08-0.25\right)$ and De Nooijer et al. (2007) ( $\left.\mathrm{D}_{\mathrm{Cu}}=0.1-0.4\right)$ is lower than $\mathrm{D}_{\mathrm{Cu}}$ from this study (A. aomoriensis $\mathrm{D}_{\mathrm{Cu}}=1.18$, A. batava $\mathrm{D}_{\mathrm{Cu}}=0.60-4.35$, E. excavatum $\mathrm{D}_{\mathrm{Cu}}=0.95-5.67$ ). Only the lowest values of this study are in the same order of magnitude. These differences could arise from the lower concentration of $\mathrm{Cu}$ in this study or from the mixture of metals. It is also reported, that the exposure to more than one metal can cause an increased uptake of another metal into the cell (Archibald and Duong, 1984; MartinezFinley et al., 2012; Bruins et al., 2000; Shafiq et al., 1991). If more $\mathrm{Cu}$ is taken up into the cell, well may be that after the usage of $\mathrm{Cu}$ as micro-nutrient, more $\mathrm{Cu}$ is left over and is actively deposited into the calcite. It is therefore conceivable that one particular metal in our study was effecting a co-uptake of $\mathrm{Cu}$, which lead to an elevated incorporation into the calcite as compared to other studies.

The non - essential elements $\mathrm{Hg}, \mathrm{Cd}$ and $\mathrm{Pb}$ are not used in physiological processes and are therefore believed to have a higher toxic potential (Barbier et al., 2005; Raikwar et al, 2008; Ali and Khan, 2019). This in turn lead to the assumption, that these metals are incorporated into the foraminiferal cell to a smaller amount. This could also result in an enhanced removal of these metals, because they are potentially harmful for marine life and could trigger mechanisms that prevent the foraminifera from intoxication as reported for various organisms (benthic foraminifera: Bresler and Yanko, 1995; Yeast: Adle et al., 2007; Bacteria: Shaw and Dussan, 2015; Microalgae: Duque et al., 2019). Furthermore, it could increase the input of these metals into the foraminiferal calcite as a further removing mechanism. The ionic radii of $\mathrm{Pb}^{2+}$ in calcite-coordination is $1.19 \AA$, which is remarkably higher than those of $\mathrm{Hg}^{2+}(1.02 \AA)$ and $\mathrm{Cd}^{2+}(0.95 \AA)$, which are comparable to $\mathrm{Ca}$ (Fig. 5). This similarity should also favour the incorporation of $\mathrm{Cd}$ and $\mathrm{Hg}$ into calcite, which holds only partly true, as $\mathrm{Cd}$ shows no trends, but $\mathrm{Hg}$ correlates in A. batava and in E. excavatum if phase 3 is not integrated into the calculations. This indicated that the incorporation of $\mathrm{Cd}$ is not straight-forward and is indeed depending on more complex factors than seawater concentration of $\mathrm{Cd}$. Nevertheless, $\mathrm{Cd}$ is incorporated well above control level in all three species. Because the ionic radius of $\mathrm{Pb}$ is bigger than that of $\mathrm{Ca}$ a smaller degree of $\mathrm{Ca}^{2+}$ substitution following the ionic radius to charge ratio theory after Rimstidt et al. (1998) is expected. This is not the case as $\mathrm{Pb}$ emerged as a well-behaved proxy. All three species incorporated $\mathrm{Pb}$ with a significant positive trend indicating that the main controlling factor is the seawater concentration of $\mathrm{Pb}$ (Fig. 4, Table 4). Calculated $\mathrm{D}_{\mathrm{Pb}}$ values including phase 3 of this study are around 1 for E. excavatum $\left(\mathrm{D}_{\mathrm{Pb}}=0.91\right)$, but lower for A. aomorienis $\left(\mathrm{D}_{\mathrm{Pb}}=0.39\right)$ and A. batava $\left(\mathrm{D}_{\mathrm{Pb}}=0.52\right)$. When excluding phase 3, $\mathrm{D}_{\mathrm{Pb}}$ values of E. excavatum $\left(\mathrm{D}_{\mathrm{Pb}}=2.0\right)$ and A. aomoriensis $\left(\mathrm{D}_{\mathrm{Pb}}=1.6\right)$ are getting even higher, which is may connected to the overload effect. Comparing the $\mathrm{D}_{\mathrm{Pb}}$ around 8.4 determined by Sagar et al. (2021), who cultured the large benthic foraminifer Amphisorus hemprichii, a symbiont-bearing, miliolid species, with varying $\mathrm{Pb}$ concentrations $\left(0.5-80 \mu \mathrm{m} \mathrm{l}^{-1}\right)$ that are comparable to our concentration range $\left(\sim 0.11-30 \mu \mathrm{m} \mathrm{l}^{-1}\right)$, the $\mathrm{D}_{\mathrm{Pb}}$ of this study are a little lower. Nevertheless, $\mathrm{D}_{\mathrm{Pb}}$ values from the present study are partly higher in individual phases $(A$. 
aomorienis $\mathrm{D}_{\mathrm{Pb}}$ : individual phases without phase $3=1.3-5.14$; A. batava $\mathrm{D}_{\mathrm{Pb}}$ : Control System Phase $3=2.52 ; E$. excavatum $\mathrm{D}_{\mathrm{Pb}}$ : Control System Phase 0-2 and Metal System Phase $1 \mathrm{DPb}_{\mathrm{Pb}} \geq 3$ ) and thereby match the values from Sagar et al. (2021) better. For $\mathrm{Hg}$, no partition coefficients are published so far. $\mathrm{D}_{\mathrm{Cd}}$ values from different studies ranged between 0.7 and 4 (Havach et al., 2001; Tachikawa and Elderfield, 2002; Maréchal-Abram et al., 2004) and are overall lower than $\mathrm{D}_{\mathrm{Cd}}$ values from the present study (A. aomoriensis $\mathrm{D}_{\mathrm{Cd}}=0.07-18.49$, A. batava $\mathrm{D}_{\mathrm{Cd}}=$ 0.08-14.42, E. excavatum $\left.\mathrm{D}_{\mathrm{Cd}}=0.06-49.45\right)$, but mean $\mathrm{D}_{\mathrm{Cd}}$ values are of the same order of magnitude. Cd is known to mimic other metals that are essential to diverse biological functions (Martelli et al., 2006; Urani et al., 2015; Losada Ros et al., 2020). Consequently, one may argue that cadmium follows a Trojan horse strategy to get assimilated (Martelli et al., 2006), which could also be the case for Hg. Smith et al. (2020) found a strong correlation between the $\mathrm{Cd}$ concentration in the seawater and the foraminiferal shell in the species Haynesina germanica, Ammonia tepida, Quinqueloculina sabulosa and Triloculina oblonga. This trend was not present in our study, as no species showed a covariance (Fig. 4, Table 4) indicating the influence of more complex mechanisms than simple incorporation of the seawater ion concentration. Investigations on deep water benthic foraminifera like Cibicidoides wuellerstorfi, Uvigerina peregrine and Melonis barleeanum revealed a smaller range in the $D_{C d}$ values (e.g., McCorkle et al., 1995; Tachikawa and Elderfield, 2002), which most likely originated from the smaller variability of the Cd concentration in their seawater as compared to this study.

The importance of other metals like $\mathrm{Sn}, \mathrm{Cr}, \mathrm{Ag}$ and $\mathrm{Ni}$ is not fully understood yet but some of them are believed to have certain biological functions in the cells of animals or plants (Horovitz, 1988; Mertz, 1993; Lukaski, 1999; Pilon-Smits et al., 2009; Hänsch \& Mendel, 2009; Chen et al., 2009). For example, Ni is important for plants and bacteria (Poonkothai and Vijayavathi, 2012; Maret, 2016). The ionic radii of these metals in calcite-coordination is rather different $(\mathrm{Sn}=1.18 \AA$; $\mathrm{Ag}=1.15 \AA$; $\mathrm{Cr}=0.62 \AA ; \mathrm{Ni}=0.69 \AA$ ) and deviate from the ionic radius of calcite, too (Fig. 5).

Ni was incorporated with a positive trend in E. excavatum, but with no clear trend in the Ammonia species (Fig. 4 , Table 4). Munsel et al. (2010) found a similar trend in A. tepida and their calculated $\mathrm{D}_{\mathrm{Ni}}$ values $\left(\mathrm{D}_{\mathrm{Ni}}=0.4-2.0\right)$ are in good agreement with our results when the highest metal phase of this study is not taken into account $(A$. aomoriensis $\mathrm{D}_{\mathrm{Ni}}=0.19-0.94$, A. batava $\mathrm{D}_{\mathrm{Ni}}=0.15-0.41$, E. excavatum $\mathrm{D}_{\mathrm{Ni}}=0.64-1.35$ ). Ag exhibited a strong positive correlation between seawater and foraminiferal shell in all three foraminiferal species. Partition coefficients for $\mathrm{Ag}\left(\right.$ A. aomoriensis $\mathrm{D}_{\mathrm{Ag}}=0.56$, A. batava $\mathrm{D}_{\mathrm{Ag}}=0.17$, E. excavatum $\left.\mathrm{D}_{\mathrm{Ag}}=0.47\right)$ cannot be compared to other studies as no literature is available, but the general trend, the ionic radius and the $\mathrm{D}_{\mathrm{TE}}$ values are comparable to other elements in this study e.g., to $\mathrm{Pb}$. $\mathrm{Ag}$ and $\mathrm{Ni}$ both display a well-behaved proxy for the estimation of seawater concentration of these ions (Fig. 4, Table 4).

$\mathrm{Cr}$ and $\mathrm{Sn}$, on the other hand, were not incorporated in a higher amount when the concentration of these metals in the culturing medium was raised, except for $\mathrm{Cr}$ in E. excavatum, which showed a positive correlation. The $\mathrm{D}_{\mathrm{Cr}}$ values presented in Remmelzwaal et al. (2019) $\left(\mathrm{D}_{\mathrm{Cr}}>107\right)$, based on the tropical, symbiont bearing foraminifera Amphistegina spp., are at least one order of magnitude higher than $\mathrm{D}_{\mathrm{Cr}}$ values in this study $\left(\right.$ A a amoriensis $\mathrm{D}_{\mathrm{Cr}}=$ 0.74-10.3, A. batava $\mathrm{D}_{\mathrm{Cr}}=0.4-6.8$, E. excavatum $\mathrm{D}_{\mathrm{Cr}}=2.1$ ). For $\mathrm{Sn}$, no comparative studies are available. One possible reason for dynamics of $\mathrm{Cr}$ are the comparable low concentrations in the culturing medium and furthermore, the differences between the phases are also very low (Fig. 3, Fig. B1 and Table 3). It may be that the concentration of $\mathrm{Cr}$ needs to be further elevated and the concentration range needs to be extended before the foraminifera are able to incorporate $\mathrm{Cr}$ with significant differences between concentrations. We may speculate that 
the same could apply for Sn. Besides, Remmelzwaal et al. (2019) suggested, that $\mathrm{Cr}$ in foraminiferal shells is mainly a result of post-depositional overprinting. Diagenetic processes are very unlikely to play a role in our experiments, which would explain, why we do not recognise a correlation between the concentration of $\mathrm{Cr}$ in the culturing medium and in foraminiferal calcite.

\subsection{Interspecies variability}

The three different species cultured in this study clearly incorporate the same metal in different ways, which is most visible in the overall higher TE/Ca values of E. excavatum compared to species from the genus Ammonia (Fig. 4, Table 4). Koho et al. (2017) suggested that these differences in the incorporation result from different microhabitats used by different foraminiferal species. This might be true in nature. In our experiments, however, the sediment in the cavities was only a few mm thick and no redox horizon was recognised when recovering the foraminifera after the experiment. Therefore, all foraminifera were living in the same microhabitat.

Another possible reason for the difference between E. excavatum and Ammonia species is their nutrition strategy. As discussed above, $\mathrm{D}_{\mathrm{TE}}$ values were markedly higher in symbiont-bearing species. Ammonia species do not harbour endosymbionts (Jauffrais et al., 2016), whereas at least five intertidal Elphidium species were husbanding diatom chloroplasts, including E. excavatum (e.g., Pillet et al., 2011; Cesborn et al., 2017). However, an earlier study could not corroborate the assumption that Elphidium species living at greater water depth in the Baltic Sea may contain endosymbiontic zooxantellae (Schönfeld and Numberger, 2007). In our experiment, dead Nannochloropsis were fed, which is certainly not the preferred food source for E. excavatum (Pillet et al., 2011). This could lead to a slower growth and E. excavatum built on average only 1 chamber during the individual culturing period of 21 days while Ammonia species built more than four chambers. Furthermore, E. excavatum did not reproduce, even though the culturing period is close to the generation time of this species (Haake, 1962). When growth is slower it could be possible that a higher amount of a metal is incorporated into the shell, which would lead to higher TE/Ca values in this species. Another possibility is the timing of chamber formation. As $E$. excavatum formed on average one new chamber, it is possible that this chamber was formed during the high peak in the metal concentration during the beginning of the culturing phases (Fig. B1, Table A1). This could in turn lead to a higher uptake of the metals and higher $\mathrm{D}_{\mathrm{TE}}$ values. Both Ammonia species on the other hand, formed more chambers, which makes it most likely that not only the first high concentration influences their overall $\mathrm{D}_{\mathrm{TE}}$ value. Unfortunately, it is not possible to constrain exactly when the specimens formed their new chambers.

\subsection{Application of TE/Ca values in the foraminiferal shell}

Table 5: Comparison of the heavy metal concentrations in different regions of the world to values used for the culturing experiments in this study. It is indicated whether the values of this study are comparable to environmental values or if values from this study are higher or lower. (EPA = Environmental Protection Agency, USA)

\begin{tabular}{llccl}
\hline Element & Study area & $\begin{array}{c}\text { Concentration } \\
\text { in } \boldsymbol{\mu g ~ l}^{\mathbf{1}}\end{array}$ & Comparable? & Reference \\
\hline \multirow{2}{*}{$\mathbf{A g}$} & \multirow{2}{*}{ EPA Recommended Values (acute) } & $0.06-4.61$ & & This study \\
\hline
\end{tabular}




\begin{tabular}{|c|c|c|c|c|}
\hline & $\begin{array}{l}\text { Restronguet Creek, U.K. + Adriatic } \\
\text { Sea }\end{array}$ & $0.0025-0.03$ & yes & Barriada et al., 2007 \\
\hline & Ibaraki coast + Watarase river & $0.014-0.03$ & yes & Shijo et al., 1989 \\
\hline \multirow{13}{*}{ Cd } & & $0.14-30.61$ & & This study \\
\hline & EPA Recommended Values (chronic) & 7.9 & yes & Prothro, 1993 \\
\hline & Suva, Fiji & $150-250$ & no, low & Arikibe and Prasad, 2020 \\
\hline & Black Sea in Rize, Turkey & $1-3$ & yes & Baltas et al., 2017 \\
\hline & Gulf of Chabahar, Oman Sea & $0.15-0.19$ & yes & Bazzi, 2014 \\
\hline & Gulf of Kutch, Arabian Sea & $200-1580$ & no, low & Chakraborty et al., 2014 \\
\hline & $\begin{array}{l}\text { East London + Port Elizabeth } \\
\text { harbours, } \\
\text { U.K. }\end{array}$ & $200-72600$ & no, low & $\begin{array}{l}\text { Fatoki and Mathabatha, } \\
2001\end{array}$ \\
\hline & Yalujiang Estuary, China & $0.83-1.33$ & yes & Li et al., 2017 \\
\hline & Gulf San Jorge, Argentina & 0.01-0.09 & yes & Muse et al., 1999 \\
\hline & $\begin{array}{l}\text { Alang-Sosiya ship scrapping yard, } \\
\text { Gulf of Cambay, India }\end{array}$ & $34-560$ & yes & Reddy et al., 2005 \\
\hline & Kamal estuary, Jakarta & $0.01-0.02$ & no, high & Putri et al., 2012 \\
\hline & Jakarta Bay & $0.04-0.104$ & yes & Williams et al., 2000 \\
\hline & Kepez harbor of Canakkale, Turkey & $19-73800$ & yes & $\begin{array}{l}\text { Y1lmaz and Sadikoglu, } \\
2011\end{array}$ \\
\hline \multirow{8}{*}{$\mathbf{C r}$} & & $0.1-14.0$ & & This study \\
\hline & EPA Recommended Values (chronic) & 50 & no, low & Prothro, 1993 \\
\hline & Gulf of Chabahar, Oman Sea & 20.16-21.46 & yes & Bazzi, 2014 \\
\hline & Gulf of Kutch, Arabian Sea & $260-3010$ & no, low & Chakraborty et al., 2014 \\
\hline & Yalujiang Estuary, China & $0.113-0.14$ & yes & Li et al., 2017 \\
\hline & Gulf San Jorge, Argentina & $0.04-0.5$ & yes & Muse et al., 1998 \\
\hline & Jakarta Bay & $0.511-5.25$ & yes & Williams et al., 2000 \\
\hline & $\begin{array}{l}\text { Alang-Sosiya ship scrapping yard, } \\
\text { Gulf of Cambay, India }\end{array}$ & $35-765$ & no, low & Reddy et al., 2005 \\
\hline \multirow{11}{*}{$\mathbf{C u}$} & & $0.6-6.2$ & & This study \\
\hline & EPA Recommended Values (chronic) & 3.1 & yes & Prothro, 1993 \\
\hline & Suva, Fiji & $880-10290$ & no, low & Arikibe and Prasad, 2020 \\
\hline & Black Sea in Rize, Turkey & $30-242$ & no, low & Baltas et al., 2017 \\
\hline & Gulf of Chabahar, Oman Sea & $3.37-5.74$ & yes & Bazzi, 2014 \\
\hline & Gulf of Kutch, Arabian Sea & $1350-1850$ & no, low & Chakraborty et al., 2014 \\
\hline & $\begin{array}{l}\text { East London + Port Elizabeth } \\
\text { harbours, U.K. }\end{array}$ & $500-42600$ & no, low & $\begin{array}{l}\text { Fatoki and Mathabatha, } \\
2001\end{array}$ \\
\hline & Yalujiang Estuary, China & $1.8-4.7$ & yes & Li et al., 2017 \\
\hline & Gulf San Jorge, Argentina & $0.02-0.65$ & yes & Muse et al., 1998 \\
\hline & Jakarta Bay & $0.405-4.04$ & yes & Williams et al., 2000 \\
\hline & $\begin{array}{l}\text { Alang-Sosiya ship scrapping yard, } \\
\text { Gulf of Cambay, India }\end{array}$ & $32-3939$ & yes & Reddy et al., 2005 \\
\hline \multirow{7}{*}{$\mathbf{H g}$} & & $0.00035-0.273$ & & This study \\
\hline & EPA Recommended Values (chronic) & 0.94 & yes & Prothro, 1993 \\
\hline & South Florida Estuaries & $0.0034-0.0074$ & yes & Kannan et al., 1998 \\
\hline & $\begin{array}{l}\text { Guadalupe River and San Francisco } \\
\text { Bay, California }\end{array}$ & $0.0017-0.135$ & yes & Thomas et al., 2002 \\
\hline & Vembanad, India & $0.0024-0.206$ & yes & Ramasamy et al., 2017 \\
\hline & Kamal estuary, Jakarta & $0.1-0.2$ & yes & Putri et al., 2011 \\
\hline & Yalujiang Estuary, China & $0.006-0.049$ & yes & Li et al., 2017 \\
\hline Mn & & $320-549$ & & This study \\
\hline
\end{tabular}




\begin{tabular}{|c|c|c|c|c|}
\hline & Black Sea in Rize, Turkey & $3-14$ & yes & Baltas et al., 2017 \\
\hline & Gulf of Chabahar, Oman Sea & $15.43-24.76$ & no, high & Bazzi, 2014 \\
\hline & Gulf of Kutch, Arabian Sea & $13000-18000$ & no, low & Chakraborty et al., 2014 \\
\hline & $\begin{array}{l}\text { East London + Port Elizabeth } \\
\text { harbours, U.K. }\end{array}$ & $300-23900$ & yes & $\begin{array}{l}\text { Fatoki and Mathabatha, } \\
2001\end{array}$ \\
\hline & $\begin{array}{l}\text { Alang-Sosiya ship scrapping yard, } \\
\text { Gulf of Cambay, India }\end{array}$ & $31-4920$ & yes & Reddy et al., 2005 \\
\hline \multirow{8}{*}{$\mathbf{N i}$} & & $2.3-24.3$ & & This study \\
\hline & EPA Recommended Values (chronic) & 8.2 & yes & Prothro, 1993 \\
\hline & Suva, Fiji & $230-800$ & no, low & Arikibe and Prasad, 2020 \\
\hline & Black Sea in Rize, Turkey & $0.006-0.036$ & yes & Baltas et al., 2017 \\
\hline & Gulf of Chabahar, Oman Sea & $16.42-17.14$ & yes & Bazzi, 2014 \\
\hline & Gulf of Kutch, Arabian Sea & $190-330$ & no, low & Chakraborty et al., 2014 \\
\hline & Jakarta Bay & $0.058-5.25$ & yes & Williams et al., 2000 \\
\hline & $\begin{array}{l}\text { Alang-Sosiya ship scrapping yard, } \\
\text { Gulf of Cambay, India }\end{array}$ & $32-944$ & yes & Reddy et al., 2005 \\
\hline \multirow{13}{*}{$\mathbf{P b}$} & & $0.11-28.35$ & & This study \\
\hline & EPA Recommended Values (chronic) & 5.6 & yes & Prothro, 1993 \\
\hline & Suva, Fiji & $880-1770$ & no, low & Arikibe and Prasad, 2020 \\
\hline & Black Sea in Rize, Turkey & $6-130$ & yes & Baltas et al., 2017 \\
\hline & Gulf of Chabahar, Oman Sea & $4.24-4.25$ & yes & Bazzi, 2014 \\
\hline & Gulf of Kutch, Arabian Sea & $20-120$ & yes & Chakraborty et al., 2014 \\
\hline & $\begin{array}{l}\text { East London + Port Elizabeth } \\
\text { harbours, U.K. }\end{array}$ & $600-16300$ & no, low & $\begin{array}{l}\text { Fatoki and Mathabatha, } \\
2001\end{array}$ \\
\hline & Yalujiang Estuary, China & $0.4-1.8$ & yes & Li et al., 2017 \\
\hline & Gulf San Jorge, Argentina & $0.1-0.5$ & yes & Muse et al., 1998 \\
\hline & $\begin{array}{l}\text { Alang-Sosiya ship scrapping yard, } \\
\text { Gulf of Cambay, India }\end{array}$ & $30-2036$ & yes & Reddy et al., 2005 \\
\hline & Kamal estuary, Jakarta & $1.3-4$ & yes & Putri et al., 2011 \\
\hline & Jakarta Bay & $0.485-3.62$ & yes & Williams et al., 2000 \\
\hline & Kepez harbor of Canakkale, Turkey & $49-9390$ & yes & $\begin{array}{l}\text { Y1lmaz and Sadikoglu, } \\
2011\end{array}$ \\
\hline \multirow{3}{*}{ Sn } & & $0.86-3.95$ & & This study \\
\hline & $\begin{array}{l}\text { estuarine seawater, Galicia Coast, } \\
\text { Spain }\end{array}$ & $0.53-1.23$ & yes & $\begin{array}{l}\text { Bermejo-Barrera et al., } \\
1999\end{array}$ \\
\hline & U.S. and European rivers & $0.0001-0.1$ & yes & Byrd and Andreae, 1982 \\
\hline \multirow{11}{*}{ Zn } & & $30.0-226.9$ & & This study \\
\hline & EPA Recommended Values (chronic) & 81 & yes & Prothro, 1993 \\
\hline & Suva, Fiji & $80-1450$ & yes & Arikibe and Prasad, 2020 \\
\hline & Black Sea in Rize, Turkey & $38-178$ & yes & Baltas et al., 2017 \\
\hline & Gulf of Chabahar, Oman Sea & $18.01-22.62$ & yes & Bazzi, 2014 \\
\hline & Gulf of Kutch, Arabian Sea & $11000-31000$ & no, low & Chakraborty et al., 2014 \\
\hline & $\begin{array}{l}\text { East London + Port Elizabeth } \\
\text { harbours, U.K. }\end{array}$ & $500-27600$ & yes & $\begin{array}{l}\text { Fatoki and Mathabatha, } \\
2001\end{array}$ \\
\hline & Yalujiang Estuary, China & $9.2-19.6$ & yes & Li et al., 2017 \\
\hline & Gulf San Jorge, Argentina & $0.01-0.55$ & no, high & Muse et al., 1998 \\
\hline & Jakarta Bay & $2-30.1$ & yes & Williams et al., 2000 \\
\hline & $\begin{array}{l}\text { Alang-Sosiya ship scrapping yard, } \\
\text { Gulf of Cambay, India }\end{array}$ & $33-5832$ & yes & Reddy et al., 2005 \\
\hline
\end{tabular}


During the past years, many studies were performed to assess the pollution level of seawater. The range of heavy metal concentrations in the culturing medium of this study are compared to the metal concentrations in polluted environments measured over the past 40 years in different regions all over the world (Table 5). The data demonstrate that the concentration range from our study is in good agreement with threatened environments in San Francisco Bay, California (Thomas et al., 2002), the Black Sea, Turkey (Baltas et al., 2017), the Gulf of Chabahar, Oman Sea (Bazzi, 2014), the Restronguet Creek, U.K., the Adriatic Sea (Ag; Barriada et al., 2007), the Yalujiang Estuary, China (Li et al., 2017), the Gulf of San Jorge, Argentina (Muse et al., 1999), Vembanad and the Gulf of Cambay, India (Ramasamy et al., 2017; Reddy et al., 2005), Kepez harbor of Canakkale, Turkey (Yilmaz and Sadikoglu, 2011), Jakarta (Williams et al., 2000; Putri et al., 2012) and with polluted U.S. and European rivers (Byrd and Andreae, 1982; Kannan et al., 1998; Thomas et al., 2002). Furthermore, the maximum metal concentration as recommended by the EPA is the lower boundary of the concentration range from this study (Prothro, 1993). A lower concentration than the EPA value is also covered by our study during the control phase or in the control system. This enables us to assess metal levels at the very beginning of the harmful pollution phase in environments, which could be used as an early-warning system for the ecological status of an area (Sagar et al., 2021). Furthermore, it allows to assess the effectiveness of contamination reducing measures. This advantage will be important in the future for the possibility to intervene or to apply more promising measures within an adequate time frame. In some regions of the world, seawater heavy metal concentrations are higher than in this study. Examples are Suva, Fiji (Arikibe and Prasad, 2020), the Gulf of Kutch, Arabian Sea (Chakraborty et al., 2014) or the East London and Port Elizabeth harbours, U.K. (Fatoki and Mathabatha, 2001) (Table 5). These areas seem to be extremely polluted, which would make it necessary to apply a higher metal concentration to the cultured foraminifera if a reconstruction covering these values should be made. However, this study clearly indicates a reduced uptake of metals of interest, when the concentration of these metals in the seawater is exceeding a certain threshold value (here between phase 2 and 3). This will make it generally difficult to model extreme high pollution levels. Indeed, it is possible to distinguish between a heavy and a moderate pollution level. Overall, the concentration of heavy metals in seawater should be decreasing all over the world due to a rigorous legislation for reduction of the heavy metal input into the environment, and due to various emission reducing measures that are applied already. This means that the concentration range of metals covered by this study is adequate for future research and monitoring of polluted systems.

\section{Conclusion}

The aim of this study was to assess the incorporation of heavy metals into the foraminiferal calcite as a function of their concentration in the seawater the foraminifera calcified in. Culturing experiments with different foraminiferal species (A. aomoriensis, A. batava and E. excavatum) that were exposed to a mixture of ten different metals $(\mathrm{Cr}, \mathrm{Mn}, \mathrm{Ni}, \mathrm{Cu}, \mathrm{Zn}, \mathrm{Ag}, \mathrm{Cd}, \mathrm{Sn}, \mathrm{Hg}$ and $\mathrm{Pb}$ ) at varying concentrations (Table 3, Fig. 3, Fig. B1) were carried out to gain further insights into the uptake of heavy metals. Laser ablation ICP-MS analysis of the newly formed calcite revealed species-specific differences in the incorporation of heavy metals. Nevertheless, all metals used in this study were incorporated into the foraminiferal calcite of all three species (Fig. 4, Table 4). Some elements showed a behaviour inferring that the uptake of these metals mainly depends on its concentration in seawater, which was indicated by strong positive correlations between the metal concentration in seawater and in the foraminiferal calcite. All three species showed a strong positive correlation between $\mathrm{Pb}$ and $\mathrm{Ag}$ in the water 
and their calcite. A. aomoriensis further revealed a slightly weaker correlation for $\mathrm{Mn}$ and $\mathrm{Cu}$. A. batava holds a strong positive correlation for $\mathrm{Mn}$ and $\mathrm{Hg}$. E. excavatum depicts a strong positive correlation for $\mathrm{Cr}$ and $\mathrm{Ni}$. Other elements like $\mathrm{Cd}$ and $\mathrm{Zn}$ showed a more complicated behaviour indicating that factors other than seawater concentration are effecting the uptake of these metals, which is demonstrated by no clear correlation between seawater and calcite metal values. The reasons for this different behaviour are yet unclear. $\mathrm{D}_{\mathrm{TE}}$ values of Ni, $\mathrm{Zn}$, $\mathrm{Cd}, \mathrm{Hg}$ and $\mathrm{Pb}$ decrease with increasing metal concentration in the seawater, which is most prominent in the highest metal treatment in all species. This could be due to an overload effect arising when the concentration of the metals is exceeding a certain threshold and could potentially be harmful or even lethal for the organism. This in turn could lead to a removal or a prevention from uptake of the metal (Nardelli et al., 2016). The results of this study facilitate a reconstruction of the heavy metal concentration in seawater for those elements showing a correlation between TE/Ca ratios in calcite and seawater. Such estimates can be based on foraminiferal samples from sediment cores and recent surface sediments, and facilitate a monitoring of anthropogenic footprints on the environment today and in the past. The presented $\mathrm{D}_{\mathrm{TE}}$ 's allow a direct quantification of metal concentrations in polluted and pristine areas. The foraminiferal species considered prevail in nearly all coastal environments worldwide, except polar latitudes. This in combination provides a powerful tool for monitoring the ecosystem status in various areas of interest.

\section{Appendix}

\section{Appendix A: Additional Tables}

Table A1: TE/Caseawater values from single weeks during the culturing period of the metal system. Measurements were carried out with ICP-MS. This values are the basis for the calculations of the mean TE/Ca values in Table 3 and for figure B1.

\begin{tabular}{|c|c|c|c|c|c|c|c|c|c|c|c|c|c|}
\hline $\begin{array}{c}\text { Metal } \\
\text { System }\end{array}$ & & & $\begin{array}{c}\text { Sampling } \\
\text { date }\end{array}$ & $\mathrm{Cr} / \mathrm{Ca}$ & $\mathbf{M n} / \mathbf{C a}$ & $\mathrm{Ni} / \mathrm{Ca}$ & $\mathrm{Cu} / \mathrm{Ca}$ & $\mathrm{Zn} / \mathrm{Ca}$ & $\mathrm{Ag} / \mathrm{Ca}$ & $\mathrm{Cd} / \mathrm{Ca}$ & Sn/Ca & $\mathrm{Hg} / \mathrm{Ca}$ & $\mathrm{Pb} / \mathrm{Ca}$ \\
\hline & Phase & Day & & $\begin{array}{l}\mu \mathrm{mol} \\
\mathrm{mol}^{-1}\end{array}$ & $\begin{array}{l}\mu \mathrm{mol} \\
\mathrm{mol}^{-1}\end{array}$ & $\begin{array}{l}\mu \mathrm{mol} \\
\mathrm{mol}^{-1}\end{array}$ & $\begin{array}{l}\mu \mathrm{mol}^{-1} \\
\mathrm{~mol}^{-1}\end{array}$ & & $\begin{array}{l}\mu \mathrm{mol} \\
\mathrm{mol}^{-1}\end{array}$ & $\begin{array}{l}\mu \mathrm{mol}^{\prime} \\
\mathrm{mol}^{-1}\end{array}$ & & $\begin{array}{l}\mathrm{nmol} \\
\mathrm{mol}^{-1}\end{array}$ & $\begin{array}{l}\mu \mathrm{mol}^{-1} \\
\mathrm{~mol}^{-1}\end{array}$ \\
\hline FR0 W2 & 0 & 10 & 10.2 .20 & 12.80 & 818.54 & 7.60 & 27.75 & 100.19 & 0.16 & 0.44 & 3.20 & & 0.63 \\
\hline FR0 W3 & 0 & 17 & 19.2 .20 & 16 & 858.94 & 7.23 & 74 & 107.69 & 0.05 & 0.43 & 2.94 & 5.28 & 0.43 \\
\hline FR1 W1 & 1 & 2 & 27.2 .20 & 13.59 & 862.52 & 7.08 & 6.25 & 97.45 & 0.37 & 1.00 & 4.98 & 43.07 & 1.03 \\
\hline FR1 W2 & 1 & 9 & 5.3 .20 & 5.86 & 796.65 & 6.69 & 2.23 & 93.09 & 0.04 & 1.06 & 3.87 & 19.13 & 0.69 \\
\hline FR1 W3 & 1 & 13 & & 7.03 & 819.38 & 6.86 & 2.14 & 95.50 & 0.06 & 1.08 & 4.23 & 27.17 & 0.62 \\
\hline FR1 W4 & 1 & 20 & 16.3 .20 & 7.75 & 844.23 & 7.94 & 2.77 & 95.75 & 0.11 & 1.19 & 4.11 & 60.20 & 0.68 \\
\hline FR2 W1 & 2 & 2 & & 13.68 & 825.59 & 10.02 & & & 1.88 & 5.20 & 5.37 & & 5.70 \\
\hline FR2 W2 & 2 & 8 & & 16.49 & 820.63 & 9.75 & 2.78 & 134.85 & 0.41 & 4.96 & 5.46 & 494.26 & 3.07 \\
\hline FR2 W3 & 2 & 15 & 2.4 .20 & 13.31 & 811.64 & 9.44 & 2.23 & 132.12 & 0.31 & 4.89 & 5.10 & 287.70 & 2.50 \\
\hline FR2 W4 & 2 & 19 & & 15.47 & 789.96 & 9.77 & 2.23 & 135.50 & 0.33 & 4.75 & 5.19 & 210.66 & 2.20 \\
\hline FR3 W1 & 3 & 2 & 9.4 .20 & 52.74 & 1558.73 & 74.72 & 15.89 & 772.38 & 31.53 & 87.65 & 18.31 & 6123.75 & 125.25 \\
\hline FR3 W2 & 3 & 7 & 14.4 .20 & 39.90 & 1281.58 & 46.73 & 3.67 & 455.31 & 7.95 & 61.37 & 11.84 & & 70.27 \\
\hline FR3 W3 & 3 & 16 & 23.4 .20 & 26.97 & 1469.59 & 66.07 & 3.55 & 579.52 & 4.13 & 84.82 & 5.87 & 2858.26 & 53.51 \\
\hline FR3 W4 & 3 & 20 & 27.4 .20 & 25.59 & 1397.18 & 65.00 & 3.01 & 550.78 & 4.31 & 84.23 & 5.02 & 1640.01 & 45.72 \\
\hline
\end{tabular}


Table A2: Average concentration, $\operatorname{RSD}(1 \sigma$ in $\%)$, literature values, accuracy in comparison to literature values and number of measurements of the reference materials SLRS-6, SLEW-3, in-house reference materials (South Atlantic surface water and South Atlantic Gyre water) and NASS-6 measured with ICP-MS. Average concentration, RSD and accuracy values displayed here are averaged from single measuring days. $\mathrm{Cr}$ values are analysed after dilution of the samples and all other elements were analyses after preconcentration with a SeaFAST system. NRCC - National Research Council Canada. *Values originated from 1:10 dilution of SLRS-6.

\begin{tabular}{|c|c|c|c|c|c|c|c|}
\hline $\begin{array}{c}\text { Reference } \\
\text { Materials }\end{array}$ & $\mathrm{Cr}$ & Mn & $\mathbf{N i}$ & $\mathrm{Cu}$ & $\mathbf{Z n}$ & Cd & $\mathbf{P b}$ \\
\hline SLRS-6 & $\mathrm{nmol} \mathrm{kg}^{-1}$ & $\mathrm{nmol} \mathrm{kg}^{-1}$ & $\mathrm{nmol} \mathrm{kg}^{-1}$ & nmol kg $^{-1}$ & $\mathrm{nmol} \mathrm{kg}^{-1}$ & $\mathrm{nmol} \mathrm{kg}^{-1}$ & nmol kg-1 \\
\hline Average conc. & 4732 & 52956 & 9811 & $338014 *$ & $31391^{*}$ & 62 & 786 \\
\hline RSD $\%$ & 3.5 & 3.9 & 6.0 & $1.7^{*}$ & $7.2^{*}$ & 12.8 & 0.8 \\
\hline $\begin{array}{l}\text { Yeghicheyan } \\
\text { et al., } 2019\end{array}$ & 4509 & 38616 & 10496 & $376378^{*}$ & $26920^{*}$ & 56 & 820 \\
\hline Accuracy & 0.96 & 0.74 & 1.08 & $1.11 *$ & $0.86^{*}$ & 0.90 & 1.04 \\
\hline Number & 4 & 11 & 11 & $13^{*}$ & $13^{*}$ & 7 & 7 \\
\hline \multicolumn{8}{|l|}{ SLEW-3 } \\
\hline Average conc. & & 40007 & 17508 & 22907 & 4442 & 343 & \\
\hline RSD $\%$ & & 4.3 & 3.5 & 4.2 & 9.1 & 4.8 & \\
\hline $\begin{array}{l}\text { Leonhard } \\
\text { et al., } 2002\end{array}$ & & 29326 & 20958 & 24409 & 3074 & 427 & \\
\hline Accuracy & & 0.74 & 1.21 & 1.07 & 0.78 & 1.28 & \\
\hline Number & & 12 & 12 & 12 & 12 & 12 & \\
\hline \multicolumn{8}{|c|}{ South Atlantic Gyre water } \\
\hline Average conc. & & 1615 & 2189 & 2649 & 5614 & & \\
\hline $\mathrm{RSD} \%$ & & 6.2 & 3.7 & 5.3 & 13.2 & & \\
\hline Number & & 10 & 10 & 10 & 10 & & \\
\hline \multicolumn{8}{|c|}{ South Atlantic surface water } \\
\hline Average conc. & & 1959 & 2417 & 2646 & 39718 & & \\
\hline $\mathrm{RSD} \%$ & & 6.8 & 2.8 & 5.8 & 2.2 & & \\
\hline Number & & 6 & 6 & 6 & 6 & & \\
\hline \multicolumn{8}{|l|}{ NASS-6 } \\
\hline Average conc. & 6747 & 11162 & 3557 & 5206 & 5158 & 169 & \\
\hline $\mathrm{RSD} \%$ & 15.9 & 5.2 & 3.2 & 3.0 & 25.3 & 7.0 & \\
\hline NRCC & 2293 & 9654 & 5129 & 3528 & 3931 & 165 & \\
\hline Accuracy & 0.34 & 0.87 & 0.76 & 0.35 & 0.81 & 0.98 & \\
\hline Number & 9 & 11 & 11 & 11 & 11 & 2 & \\
\hline
\end{tabular}

Table A3: Average concentration, RSD ( $1 \sigma$ in \%), literature values, accuracy in comparison to literature values and number of measurements of the reference materials NIST SRM 610, NIST SRM 614, JCt-1, JCp-1, MACS-3 and ECRM752-1 measured with LA-ICP-MS. Please note that for the ECRM752-1 no reported values for the elements of interest are available, which is also the case for some elements in other reference materials. It is important to note that the $\mathrm{Hg} / \mathrm{Ca}$ values in the NIST glasses are not reliable as $\mathrm{Hg}$ is volatile and most likely volatilized during the glass formation. Average concentration, RSD and accuracy values displayed here are averaged from single measuring days. 
https://doi.org/10.5194/bg-2021-158

Preprint. Discussion started: 12 August 2021

(c) Author(s) 2021. CC BY 4.0 License.

\begin{tabular}{|c|c|c|c|c|c|c|c|c|c|c|}
\hline $\begin{array}{l}\text { Reference } \\
\text { materials }\end{array}$ & $\mathrm{Cr} / \mathrm{Ca}$ & $\mathrm{Mn} / \mathrm{Ca}$ & $\mathrm{Ni} / \mathrm{Ca}$ & $\mathbf{C u} / \mathbf{C a}$ & $\mathrm{Zn} / \mathrm{Ca}$ & $\mathbf{A g} / \mathbf{C a}$ & $\mathrm{Cd} / \mathrm{Ca}$ & $\mathrm{Sn} / \mathrm{Ca}$ & $\mathrm{Hg} / \mathrm{Ca}$ & $\mathrm{Pb} / \mathrm{Ca}$ \\
\hline NIST SRM 612 & $\begin{array}{l}\mathrm{mmol} \\
\mathrm{mol}^{-1}\end{array}$ & $\begin{array}{l}\mathrm{mmol} \\
\mathrm{mol}^{-1}\end{array}$ & $\begin{array}{l}\mathrm{mmol}^{-1} \\
\mathrm{~mol}^{-1}\end{array}$ & $\begin{array}{l}\mathrm{mmol} \\
\mathrm{mol}^{-1}\end{array}$ & $\begin{array}{l}\mathrm{mmol} \\
\mathrm{mol}^{-1}\end{array}$ & $\begin{array}{l}\mathrm{mmol} \\
\mathrm{mol}^{-1}\end{array}$ & $\begin{array}{l}\mathrm{mmol} \\
\mathrm{mol}^{-1}\end{array}$ & $\begin{array}{l}\mathrm{mmol} \\
\mathrm{mol}^{-1}\end{array}$ & $\begin{array}{l}\mu \mathrm{mol} \\
\mathrm{mol}^{-1}\end{array}$ & $\begin{array}{c}\mathrm{mmol} \\
\mathrm{mol}^{-1}\end{array}$ \\
\hline Mean value & 0.33 & 0.34 & 0.33 & 0.29 & 0.37 & 0.08 & 0.12 & 0.15 & 2.26 & 0.10 \\
\hline $\mathrm{RSD} \%$ & 3.49 & 2.09 & 2.30 & 4.05 & 2.97 & 5.01 & 3.90 & 2.08 & 26.32 & 2.52 \\
\hline $\begin{array}{l}\text { Jochum et al., } \\
2011\end{array}$ & 0.33 & 0.33 & 0.31 & 0.28 & 0.28 & 0.10 & 0.12 & 0.15 & & 0.09 \\
\hline Number of spots & 69 & 75 & 75 & 75 & 73 & 73 & 75 & 75 & 41 & 75 \\
\hline NIST SRM 614 & $\begin{array}{l}\mu \mathrm{mol}^{\prime} \\
\mathrm{mol}^{-1}\end{array}$ & $\begin{array}{l}\mu \mathrm{mol}^{\prime} \\
\mathrm{mol}^{-1}\end{array}$ & $\begin{array}{l}\mu \mathrm{mol} \\
\mathrm{mol}^{-1}\end{array}$ & $\begin{array}{l}\mu \mathrm{mol} \\
\mathrm{mol}^{-1}\end{array}$ & $\begin{array}{l}\mu \mathrm{mol} \\
\mathrm{mol}^{-1}\end{array}$ & $\begin{array}{l}\mu \mathrm{mol}^{\prime} \\
\mathrm{mol}^{-1}\end{array}$ & $\begin{array}{l}\mu \mathrm{mol} \\
\mathrm{mol}^{-1}\end{array}$ & $\begin{array}{l}\mu \mathrm{mol} \\
\mathrm{mol}^{-1}\end{array}$ & $\begin{array}{l}\mathrm{nmol} \\
\mathrm{mol}^{-1}\end{array}$ & $\begin{array}{l}\mu \mathrm{mol}^{-1} \\
\mathrm{~mol}^{-1}\end{array}$ \\
\hline Mean value & 19.28 & 10.31 & 8.43 & 15.86 & 67.58 & 2.13 & 15.53 & 5.97 & 20.93 & 5.23 \\
\hline $\mathrm{RSD} \%$ & 10.57 & 4.47 & 4.66 & 3.03 & 2.44 & 4.92 & 5.69 & 2.98 & 20.69 & 1.98 \\
\hline $\begin{array}{l}\text { Jochum et al., } \\
2011\end{array}$ & 10.78 & 12.18 & 8.83 & 10.16 & 20.11 & 1.83 & 2.35 & 6.67 & & 5.28 \\
\hline Accuracy & 0.57 & 1.19 & 1.06 & 0.64 & 0.30 & 0.86 & 0.23 & 1.12 & & 1.01 \\
\hline Number of spots & 35 & 38 & 37 & 39 & 38 & 38 & 38 & 39 & 19 & 39 \\
\hline MACS-3 & $\begin{array}{l}\mathrm{mmol} \\
\mathrm{mol}^{-1}\end{array}$ & $\begin{array}{l}\mathrm{mmol} \\
\mathrm{mol}^{-1}\end{array}$ & $\begin{array}{l}\mathrm{mmol} \\
\mathrm{mol}^{-1}\end{array}$ & $\begin{array}{l}\mathrm{mmol} \\
\mathrm{mol}^{-1}\end{array}$ & $\begin{array}{l}\mathrm{mmol} \\
\mathrm{mol}^{-1}\end{array}$ & $\begin{array}{l}\mathrm{mmol} \\
\mathrm{mol}^{-1}\end{array}$ & $\begin{array}{l}\mathrm{mmol} \\
\mathrm{mol}^{-1}\end{array}$ & $\begin{array}{l}\mathrm{mmol} \\
\mathrm{mol}^{-1}\end{array}$ & $\begin{array}{l}\mu \mathrm{mol}^{-1} \\
\mathrm{~mol}^{-1}\end{array}$ & $\begin{array}{l}\mathrm{mmol} \\
\mathrm{mol}^{-1}\end{array}$ \\
\hline Mean value & 0.21 & 0.97 & 0.093 & 0.17 & 0.13 & 0.065 & 0.041 & 0.042 & 5.11 & 0.026 \\
\hline $\mathrm{RSD} \%$ & 1.60 & 1.36 & 1.90 & 1.92 & 2.19 & 6.37 & 2.83 & 2.68 & 9.23 & 2.18 \\
\hline $\begin{array}{l}\text { Jochum et al., } \\
2019\end{array}$ & 0.23 & 0.99 & 0.10 & 0.19 & 0.20 & 0.054 & 0.051 & 0.049 & 5.41 & 0.031 \\
\hline Accuracy & 1.13 & 1.02 & 1.09 & 1.11 & 1.50 & 0.84 & 1.24 & 1.15 & 1.07 & 1.16 \\
\hline Number of spots & 45 & 45 & 44 & 46 & 46 & 42 & 46 & 46 & 44 & 46 \\
\hline JCt-1NP & $\begin{array}{l}\mu \mathrm{mol} \\
\mathrm{mol}^{-1}\end{array}$ & $\begin{array}{l}\mu \mathrm{mol} \\
\mathrm{mol}^{-1}\end{array}$ & $\begin{array}{l}\mu \mathrm{mol} \\
\mathrm{mol}^{-1}\end{array}$ & $\begin{array}{l}\mu \mathrm{mol} \\
\mathrm{mol}^{-1}\end{array}$ & $\begin{array}{l}\mu \mathrm{mol}^{-1} \\
\mathrm{~mol}^{-1}\end{array}$ & $\begin{array}{l}\mu \mathrm{mol}^{-} \\
\mathrm{mol}^{-1}\end{array}$ & $\begin{array}{l}\mu \mathrm{mol}^{-} \\
\mathrm{mol}^{-1}\end{array}$ & $\begin{array}{l}\mu \mathrm{mol} \\
\mathrm{mol}^{-1}\end{array}$ & $\begin{array}{l}\mathrm{nmol} \\
\mathrm{mol}^{-1}\end{array}$ & $\begin{array}{l}\mu \mathrm{mol}^{-1} \\
\mathrm{~mol}^{-1}\end{array}$ \\
\hline Mean value & 6.16 & 0.91 & 0.37 & 1.14 & 1.46 & 0.01 & 1.60 & 2.30 & 8.93 & 0.063 \\
\hline $\mathrm{RSD} \%$ & 14.25 & 15.59 & 9.56 & 7.44 & 10.37 & 6.57 & 11.75 & 5.06 & 23.95 & 5.86 \\
\hline $\begin{array}{l}\text { Jochum et al., } \\
2019\end{array}$ & 0.93 & 1.01 & 1.03 & 1.48 & & & & & & 0.064 \\
\hline Accuracy & 0.15 & 1.19 & 2.71 & 1.31 & & & & & & 1.04 \\
\hline Number of spots & 44 & 38 & 45 & 47 & 45 & 11 & 46 & 13 & 26 & 48 \\
\hline JCp-1NP & $\begin{array}{l}\mu \mathrm{mol}^{\prime} \\
\mathrm{mol}^{-1}\end{array}$ & $\begin{array}{l}\mu \mathrm{mol}^{-1} \\
\mathrm{~mol}^{-1}\end{array}$ & $\begin{array}{l}\mu \mathrm{mol} \\
\mathrm{mol}^{-1}\end{array}$ & $\begin{array}{l}\mu \mathrm{mol} \\
\mathrm{mol}^{-1}\end{array}$ & $\begin{array}{l}\mu \mathrm{mol}^{-} \\
\mathrm{mol}^{-1}\end{array}$ & $\begin{array}{l}\mu \mathrm{mol}^{\prime} \\
\mathrm{mol}^{-1}\end{array}$ & $\begin{array}{l}\mu \mathrm{mol}^{\prime} \\
\mathrm{mol}^{-1}\end{array}$ & $\begin{array}{l}\mu \mathrm{mol}^{-1} \\
\mathrm{~mol}^{-1}\end{array}$ & $\begin{array}{l}\mathrm{nmol} \\
\mathrm{mol}^{-1}\end{array}$ & $\begin{array}{l}\mu \mathrm{mol}^{-1} \\
\mathrm{~mol}^{-1}\end{array}$ \\
\hline Mean value & 9.61 & 2.11 & 0.50 & 0.84 & 1.81 & 0.02 & 0.98 & 0.06 & 8.25 & 0.13 \\
\hline $\mathrm{RSD} \%$ & 7.91 & 4.62 & 6.89 & 6.36 & 6.53 & 11.34 & 11.08 & 10.68 & 20.96 & 6.15 \\
\hline $\begin{array}{l}\text { Jochum et al., } \\
2019\end{array}$ & 1.27 & 2.16 & 1.05 & 1.29 & 3.53 & & & & & 0.15 \\
\hline Accuracy & 0.15 & 1.06 & 2.10 & 1.25 & 1.96 & & & & & 1.19 \\
\hline Number of spots & 37 & 41 & 41 & 40 & 41 & 21 & 36 & 30 & 21 & 47 \\
\hline ECRM752-1 & $\begin{array}{l}\mu \mathrm{mol}^{-1} \\
\mathrm{~mol}^{-1}\end{array}$ & $\begin{array}{l}\mu \mathrm{mol}^{-1} \\
\mathrm{~mol}^{-1}\end{array}$ & $\begin{array}{l}\mu \mathrm{mol} \\
\mathrm{mol}^{-1}\end{array}$ & $\begin{array}{l}\mu \mathrm{mol} \\
\mathrm{mol}^{-1}\end{array}$ & $\begin{array}{l}\mu \mathrm{mol} \\
\mathrm{mol}^{-1}\end{array}$ & $\begin{array}{l}\mu \mathrm{mol}^{-1} \\
\mathrm{~mol}^{-1}\end{array}$ & $\begin{array}{l}\mu \mathrm{mol}^{\prime} \\
\mathrm{mol}^{-1}\end{array}$ & $\begin{array}{l}\mu \mathrm{mol}^{-} \\
\mathrm{mol}^{-1}\end{array}$ & $\begin{array}{l}\mathrm{nmol} \\
\mathrm{mol}^{-1}\end{array}$ & $\begin{array}{l}\mu \mathrm{mol} \\
\mathrm{mol}^{-1}\end{array}$ \\
\hline Mean value & 14.75 & 144.44 & 3.87 & 2.34 & 8.40 & 0.01 & 1.54 & 0.04 & 19.14 & 0.86 \\
\hline $\mathrm{RSD} \%$ & 7.78 & 2.54 & 4.97 & 6.21 & 2.37 & 87.11 & 7.76 & 9.22 & 18.03 & 3.82 \\
\hline Number of spots & 27 & 31 & 26 & 28 & 27 & 15 & 29 & 24 & 19 & 31 \\
\hline
\end{tabular}



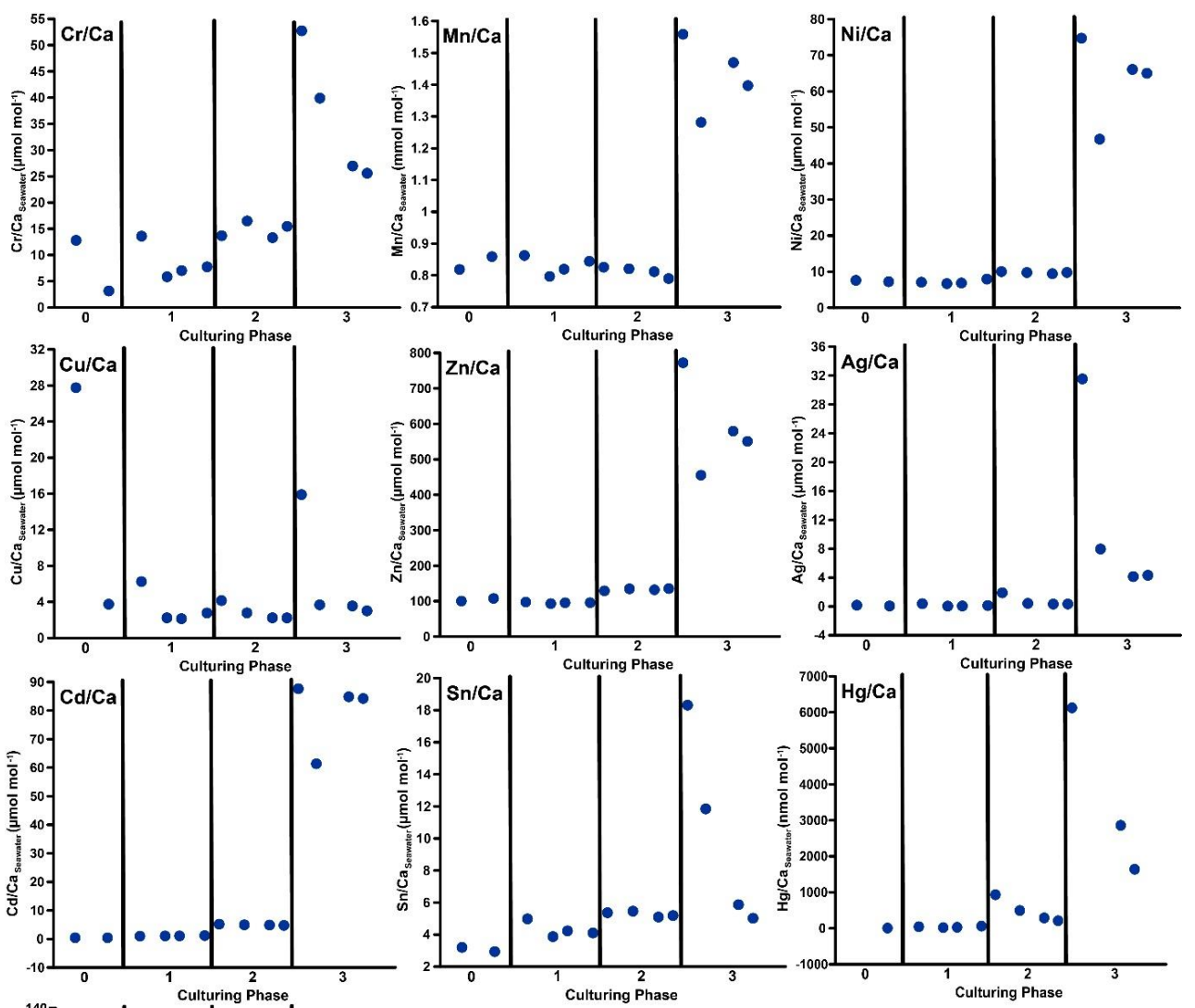

Figure B1: TE/Ca values in the culturing medium of the metal system in $\mu \mathrm{mol} \mathrm{mol}^{-1}$ or $\mathrm{nmol} \mathrm{mol}^{-1}$ divided by individual culturing phases. In this system, phase 0 is the control phase without any extra added metals and for phase 1 to 3 , the heavy metal concentration in the culturing medium was elevated. The data the figure is based on can be found in Table A1. 
https://doi.org/10.5194/bg-2021-158

Preprint. Discussion started: 12 August 2021

(c) Author(s) 2021. CC BY 4.0 License.

(i)

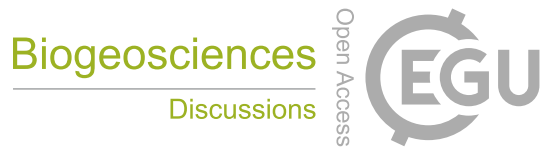
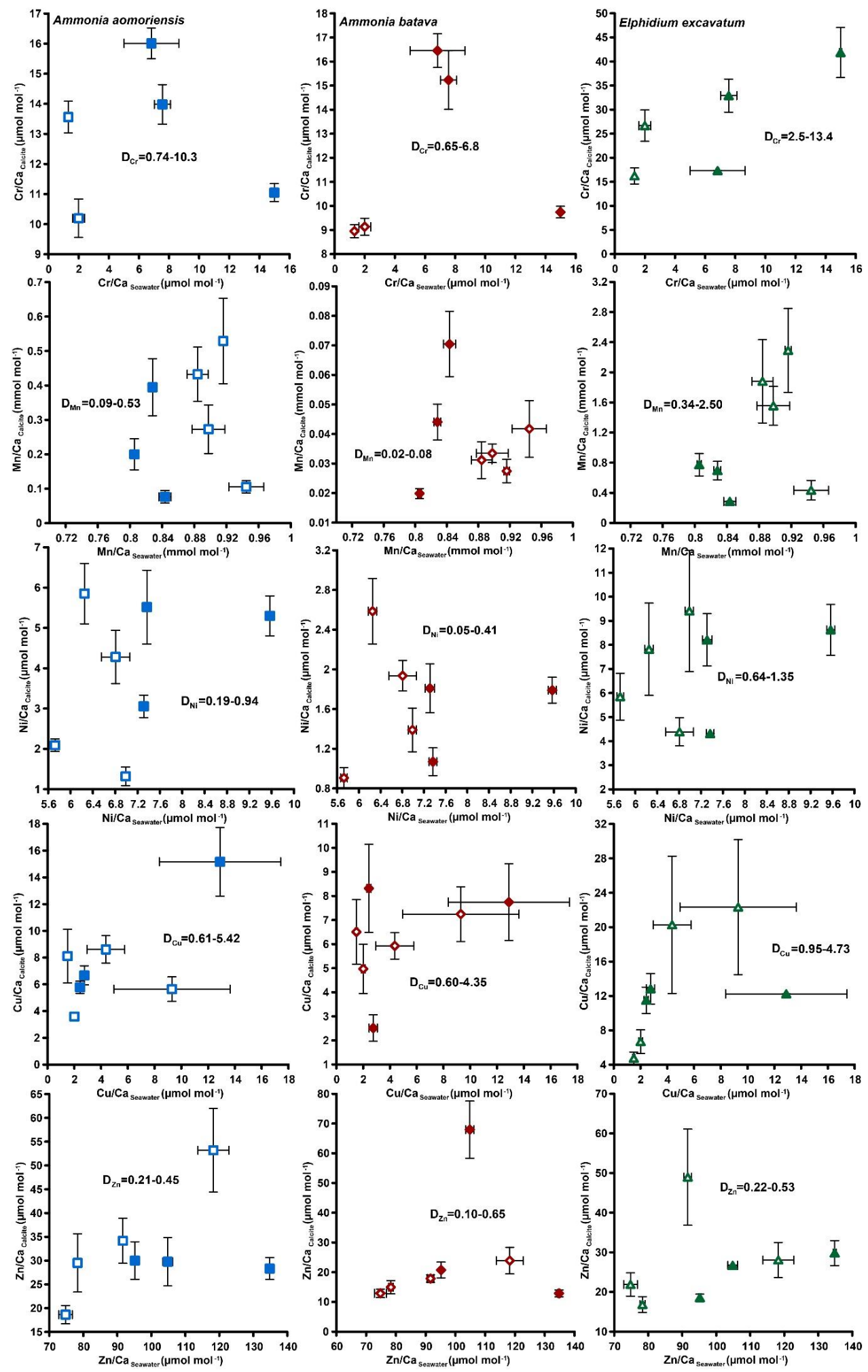

842

36 
https://doi.org/10.5194/bg-2021-158

Preprint. Discussion started: 12 August 2021

(c) Author(s) 2021. CC BY 4.0 License.
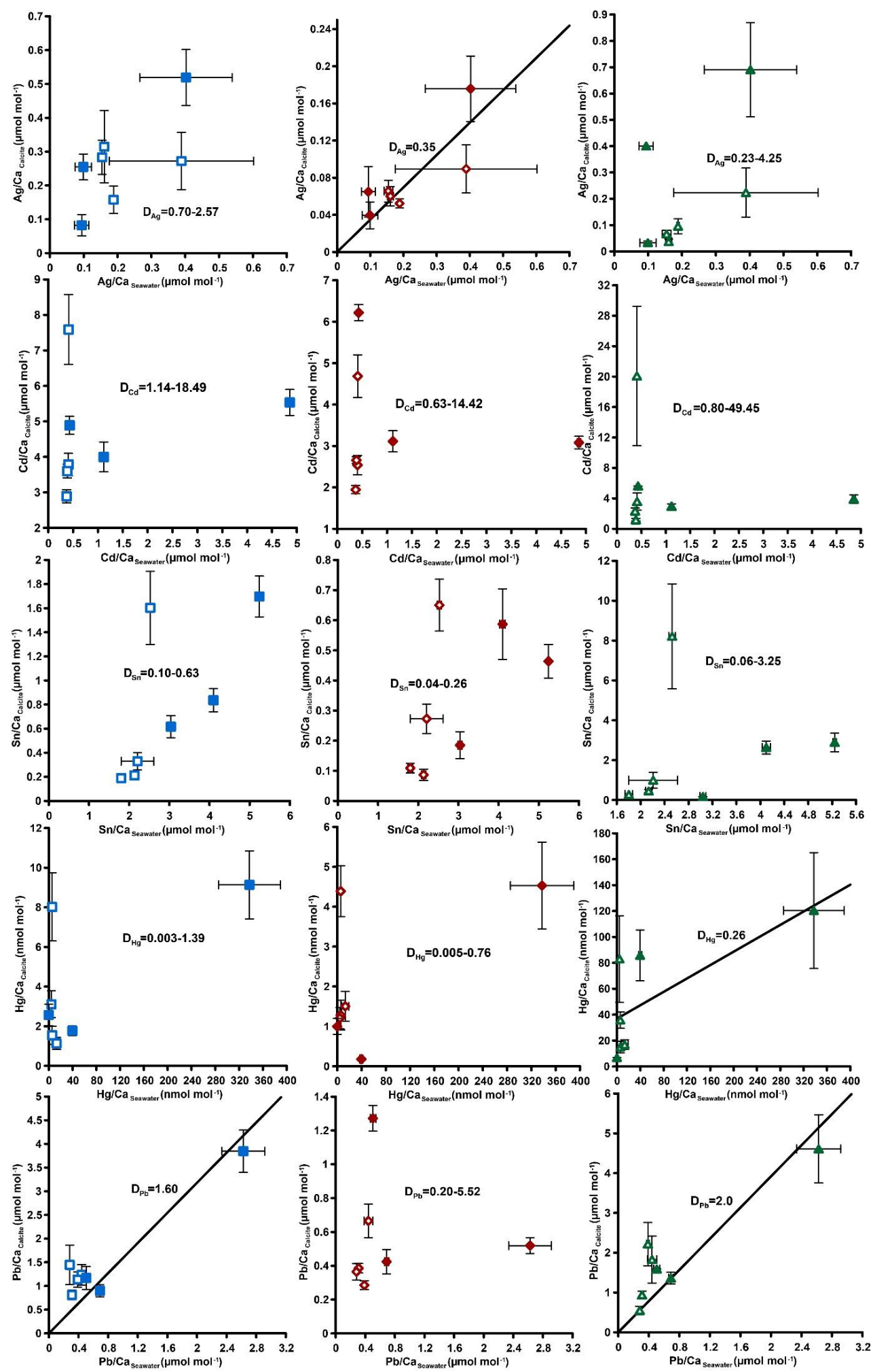


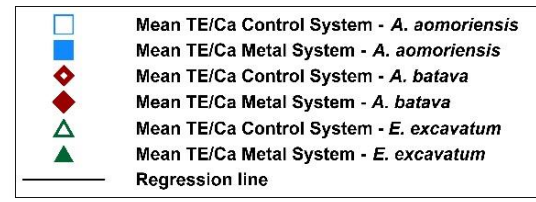

Figure B2: Mean TE/Ca values in the foraminiferal calcite versus the mean TE/Ca values in the corresponding culturing medium without phase 3 . Each data point represents the mean value of all laser ablation ICP - MS measurements on single foraminiferal chambers built up during the individual culturing phase plotted against the mean metal concentrations in the seawater averaged over the culturing phase (Table 3). Error bars symbolize the standard error of the mean. The linear regression line is based on the calculations excluding phase 3 and is only displayed when elements showed a significant correlation between seawater and calcite. D $\mathrm{D}_{\mathrm{TE}}$ 's of E. excavatum where considered without values for Phase 0 as only data from one newly formed chamber are available. All values can be found in Table 4.

\section{Supplements}

Table S1-S3: TE/CaCalcite values from Ammonia aomoriensis (Table S1), Ammonia batava (Table S2) and Elphidium excavatum (Table S3). Values represent single laser ablation spots on foraminiferal chambers that were formed during the individual culturing period in the control and the metal system. Only values above the detection limits of the individual element are presented. Furthermore, outliers are also excluded. These values are the basis for the calculation of the mean TE/Ca values in Table 4 and Fig. 4 . The sample ID indicates the species $(\mathrm{AA}=A$. aomoriensis, $\mathrm{AB}=$ A. batava, $\mathrm{E}=$ E. excavatum $)$, the culturing phase, the system $(\mathrm{R}=$ metal system, $\mathrm{L}=$ control system), the individual and the chamber that was ablated, starting from the innermost chamber going to the youngest one.

\section{Data availability}

All data generated or analysed during this study are included in this published article and its supplementary information files.

\section{Author contribution}

This study was initiated by JS and EH. SS collected the samples, cultured the foraminifera, processed the samples in the laboratory and acquired, analysed and interpreted the water and foraminiferal data. JS helped with the sampling logistics, design and implementation of the culturing experiments. EH advised and helped with the processing and analysis of the water samples and EH and DGS advised and helped with the measurements of the foraminiferal samples. SS wrote the manuscript with all the authors contributing to the discussion and data interpretation, and editing of the work.

\section{Competing interests}

The authors declare that they have no conflict of interest.

\section{Acknowledgements}

We are indebted to Tal Dagan and Alexandra-Sophie Roy, Kiel University, for providing the basic compounds of the culturing setup and for helping us with setting up the systems. Claas Hiebenthal, KIMOCC, had previously helped with the system design. Furthermore, Regina Surberg carried out the ICP-OES measurements, Kathleen 
Gosnell performed the Hg measurements in the water samples and Ulrike Westernströer set up and helped with the laser ablation measurements, which was vitally important for this manuscript. The fieldwork was supported by "Schutzstation Wattenmeer" on Hallig Hooge, in particular by a guided tour to the Japsand and by providing laboratory facilities at their station. Leif Boyens is thanked for his flexibility and his accommodation space on Hooge. The help of Danny Arndt during fieldwork is gratefully acknowledged.

\section{References}

Adle, D. J., Sinani, D., Kim, H., and Lee, J.: A cadmium-transporting P1B-type ATPase in yeast Saccharomyces cerevisiae, Journal of Biological Chemistry, 282, 947-955, doi:10.1074/jbc.M609535200, 2007.

Ali, H. and Khan, E.: Trophic transfer, bioaccumulation, and biomagnification of non-essential hazardous heavy metals and metalloids in food chains/webs - Concepts and implications for wildlife and human health, Human and Ecological Risk Assessment: An International Journal, 25, 1353-1376, doi:10.1080/10807039.2018.1469398, 2019

Alloway, B. J.: Sources of heavy metals and metalloids in soils: In: Alloway B. (eds) Heavy Metals in Soils. Environmental Pollution, Springer, Dordrecht., vol. 22, 11-50, doi:10.1007/978-94-007-4470-7_2, 2013.

Alve, E.: Benthic foraminiferal responses to estuarine pollution: A review, Journal of Foraminiferal Research, 25, 190-203, doi:10.2113/gsjfr.25.3.190, 1995.

Archibald, F. S. and Duong, M.-N.: Manganese acquisition by Lactobacillus plantarum, Journal of bacteriology, 158, 1-8, doi:10.1128/jb.158.1.1-8.1984, 1984

Arikibe, J. E. and Prasad, S.: Determination and comparison of selected heavy metal concentrations in seawater and sediment samples in the coastal area of Suva, Fiji, Marine Pollution Bulletin, 157, 111157, doi:10.1016/j.marpolbul.2020.111157, 2020.

Baltas, H., Kiris, E., and Sirin, M.: Determination of radioactivity levels and heavy metal concentrations in seawater, sediment and anchovy (Engraulis encrasicolus) from the Black Sea in Rize, Turkey, Marine Pollution Bulletin, 116, 528-533, doi:10.1016/j.marpolbul.2017.01.016, 2017.

Barbier, O., Jacquillet, G., Tauc, M., Cougnon, M., and Poujeol, P.: Effect of heavy metals on, and handling by, the kidney, Nephron Physiology, 99, p105-p110, doi:10.1159/000083981, 2005.

Barras, C., Mouret, A., Nardelli, M. P., Metzger, E., Petersen, J., La, C., Filipsson, H. L., and Jorissen, F.: Experimental calibration of manganese incorporation in foraminiferal calcite, Geochimica et Cosmochimica Acta, 237, 49-64, doi:10.1016/j.gca.2018.06.009, 2018.

Barriada, J. L., Tappin, A. D., Evans, E. H., and Achterberg, E. P.: Dissolved silver measurements in seawater, TrAC Trends in Analytical Chemistry, 26, 809-817, doi:10.1016/j.trac.2007.06.004, 2007.

Bazzi, A. O.: Heavy metals in seawater, sediments and marine organisms in the Gulf of Chabahar, Oman Sea, Journal of Oceanography and Marine Science, 5, 20-29, doi:10.5897/JOMS2014.0110, 2014.

Bé, A. W. H., Hemleben, C., Anderson, O. R., and Spindler, M.: Chamber formation in planktonic foraminifera, Micropaleontology, 25, 294-307, doi:10.2307/1485304, 1979.

Bermejo-Barrera, P., Ferrón-Novais, M., González-Campos, G., and Bermejo-Barrera, A.: Tin determination in seawater by flow injection hydride generation atomic absorption spectroscopy, Atomic Spectroscopy, 120, doi:10.46770/AS.1999.03.007, 1999. 
Bernhard, J. M., Blanks, J. K., Hintz, C. J., and Chandler, G. T.: Use of the fluorescent calcite marker calcein to label foraminiferal tests, Journal of Foraminiferal Research, 34, 96-101, doi:10.2113/0340096, 2004.

Bertlich, J., Nürnberg, D., Hathorne, E. C., de Nooijer, L. J., Mezger, E. M., Kienast, M., Nordhausen, S., Reichart, G.-J., Schönfeld, J., and Bijma, J.: Salinity control on Na incorporation into calcite tests of the planktonic foraminifera Trilobatus sacculifer-evidence from culture experiments and surface sediments., Biogeosciences, 15(20), 5991-6018, doi:10.5194/bg-2018-164, 2018.

Boltovskoy, E. and Lena, H.: Seasonal occurrences, standing crop and production in benthic Foraminifera of Puerto Deseado., Contributions from the Cushman Foundation for Foraminiferal Research, 20, 87-95, 1969.

Boyle, E. A.: Cadmium, zinc, copper, and barium in foraminifera tests, Earth and Planetary Science Letters, 53, 11-35, doi:10.1016/0012-821X(81)90022-4, 1981.

Boyle, E. A.: Chemical accumulation variations under the Peru Current during the past 130,000 years, Journal of Geophysical Research: Oceans, 88, 7667-7680, doi:10.1029/JC088iC12p07667, 1983.

Bresler, V. and Yanko, V.: Chemical ecology; a new approach to the study of living benthic epiphytic Foraminifera, Journal of Foraminiferal Research, 25, 267-279, doi:10.2113/gsjfr.25.3.267, 1995.

Bruins, M. R., Kapil, S., and Oehme, F. W.: Microbial resistance to metals in the environment, Ecotoxicology and Environmental safety, 45, 198-207, doi:10.1006/eesa.1999.1860, 2000.

Byrd, J. T. and Andreae, M. O.: Tin and methyltin species in seawater: Concentrations and fluxes, Science, 218, 565-569, doi:10.1126/science.218.4572.565, 1982.

Cang, L., Wang, Y.-j., Zhou, D.-m., and Dong, Y.-h.: Heavy metals pollution in poultry and livestock feeds and manures under intensive farming in Jiangsu Province, China, Journal of Environmental Sciences, 16, 371$374,2004$.

Cesbron, F., Geslin, E., Le Kieffre, C., Jauffrais, T., Nardelli, M. P., Langlet, D., Mabilleau, G., Jorissen, F. J., Jézéquel, D., and Metzger, E.: Sequestered chloroplasts in the benthic foraminifer Haynesina germanica: Cellular organization, oxygen fluxes and potential ecological implications, Journal of Foraminiferal Research, 47, 268-278, doi:10.2113/gsjfr.47.3.268, 2017.

Chakraborty, S., Bhattacharya, T., Singh, G., and Maity, J. P.: Benthic macroalgae as biological indicators of heavy metal pollution in the marine environments: A biomonitoring approach for pollution assessment, Ecotoxicology and Environmental safety, 100, 61-68, doi:10.1016/j.ecoenv.2013.12.003, 2014.

Chen, C., Huang, D., and Liu, J.: Functions and toxicity of nickel in plants: Recent advances and future prospects, Clean-soil, air, water, 37, 304-313, doi:10.1002/clen.200800199, 2009.

Dagan, T., Schönfeld, J., Glock, N., Roy, A.-S., Weissenbach, J., Wein, T., and Wöhle, C.: Foraminiferal nitrite respiration in oxygen minimum zones: A pilot study funded by Sonderforschungsbereich 754 Climate. Biogeochemistry Interactions in the Tropical Ocean - Initial Report Part II: Sampling, culturing and experiments with Globobulimina turgida from Gullmar Fjord, Sweden, Expedition Report, 2016.

Delaney, M. L., Bé, A. W. H., and Boyle, E. A.: Li, Sr, Mg, and Na in foraminiferal calcite shells from laboratory culture, sediment traps, and sediment cores, Geochimica et Cosmochimica Acta, 49, 1327-1341, doi:10.1016/0016-7037(85)90284-4, 1985.

Dissard, D., Nehrke, G., Reichart, G. J., and Bijma, J.: The impact of salinity on the $\mathrm{Mg} / \mathrm{Ca}$ and $\mathrm{Sr} / \mathrm{Ca}$ ratio in the benthic foraminifera Ammonia tepida: Results from culture experiments, Geochimica et Cosmochimica Acta, 74, 928-940, doi:10.1016/j.gca.2009.10.040, 2010a. 
Dissard, D., Nehrke, G., Reichart, G. J., Nouet, J., and Bijma, J.: Effect of the fluorescent indicator calcein on Mg and $\mathrm{Sr}$ incorporation into foraminiferal calcite, Geochemistry, Geophysics, Geosystems, 10, doi:10.1029/2009GC002417, 2009.

Dissard, D., Nehrke, G., Reichart, G.-J., and Bijma, J.: Impact of seawater $\mathrm{pCO}_{2}$ on calcification and $\mathrm{Mg} / \mathrm{Ca}$ and $\mathrm{Sr} / \mathrm{Ca}$ ratios in benthic foraminifera calcite: Results from culturing experiments with Ammonia tepida, Biogeosciences, 7, 81-93, doi:10.5194/bg-7-81-2010, $2010 \mathrm{~b}$.

Duque, D., Montoya, C., and Botero, L. R.: Cadmium (Cd) tolerance evaluation of three strains of microalgae of the genus Ankistrodesmus, Chlorella and Scenedesmus, Revista Facultad de Ingeniería Universidad de Antioquia, 88-95, doi:10.17533/udea.redin.20190523, 2019.

Eggins, S., Deckker, P. de, and Marshall, J.: $\mathrm{Mg} / \mathrm{Ca}$ variation in planktonic foraminifera tests: Implications for reconstructing palaeo-seawater temperature and habitat migration, Earth and Planetary Science Letters, 212, 291-306, doi:10.1016/S0012-821X(03)00283-8, 2003.

Elderfield, H.: Chromium speciation in sea water, Earth and Planetary Science Letters, 9, 10-16, doi:10.1016/0012-821X(70)90017-8, 1970.

Elderfield, H., Bertram, C. J., and Erez, J.: A biomineralization model for the incorporation of trace elements into foraminiferal calcium carbonate, Earth and Planetary Science Letters, 142, 409-423, doi:10.1016/0012821X(96)00105-7, 1996.

Erez, J.: The source of ions for biomineralization in foraminifera and their implications for paleoceanographic proxies, Reviews in mineralogy and geochemistry, 54, 115-149, doi:10.2113/0540115, 2003.

Escudero, C., Gabaldón, C., Marzal, P., and Villaescusa, I.: Effect of EDTA on divalent metal adsorption onto grape stalk and exhausted coffee wastes, Journal of Hazardouz Materials, 152, 476-485, doi:10.1016/j.jhazmat.2007.07.013, 2008.

Fatoki, O. S. and Mathabatha, S.: An assessment of heavy metal pollution in the East London and Port Elizabeth harbours, Water SA, 27, 233-240, doi:10.4314/wsa.v27i2.4997, 2001.

Flora, G., Gupta, D., and Tiwari, A.: Toxicity of lead: A review with recent updates, Interdisciplinary toxicology, 5, 47-58, doi:10.2478/v10102-012-0009-2, 2012.

Förstner, U.: Metal speciation-general concepts and applications, International Journal of Environmental Analytical Chemistry, 51, 5-23, doi:10.1080/03067319308027608, 1993.

Fricker, M. B., Kutscher, D., Aeschlimann, B., Frommer, J., Dietiker, R., Bettmer, J., and Günther, D.: High spatial resolution trace element analysis by LA-ICP-MS using a novel ablation cell for multiple or large samples, International Journal of Mass Spectrometry, 307, 39-45, doi:10.1016/j.ijms.2011.01.008, 2011.

Frontalini, F. and Coccioni, R.: Benthic foraminifera for heavy metal pollution monitoring: A case study from the central Adriatic Sea coast of Italy, Estuarine, Coastal and Shelf Science, 76, 404-417, doi:10.1016/j.ecss.2007.07.024, 2008.

Frontalini, F., Curzi, D., Giordano, F. M., Bernhard, J. M., Falcieri, E., and Coccioni, R.: Effects of lead pollution on Ammonia parkinsoniana (foraminifera): Ultrastructural and microanalytical approaches, European Journal of Histochemistry, 59, doi:10.4081/ejh.2015.2460, 2015.

Frontalini, F., Greco, M., Di Bella, L., Lejzerowicz, F., Reo, E., Caruso, A., Cosentino, C., Maccotta, A., Scopelliti, G., and Nardelli, M. P.: Assessing the effect of mercury pollution on cultured benthic foraminifera community using morphological and eDNA metabarcoding approaches, Marine Pollution Bulletin, 129, 512-524, doi:10.1016/j.marpolbul.2017.10.022, 2018a. 
Frontalini, F., Nardelli, M. P., Curzi, D., Martín-González, A., Sabbatini, A., Negri, A., Losada, M. T., Gobbi, P., Coccioni, R., and Bernhard, J. M.: Benthic foraminiferal ultrastructural alteration induced by heavy metals, Marine Micropaleontology, 138, 83-89, doi:10.1016/j.marmicro.2017.10.009, 2018b.

Gallego, A., Martín-González, A., Ortega, R., and Gutiérrez, J. C.: Flow cytometry assessment of cytotoxicity and reactive oxygen species generation by single and binary mixtures of cadmium, zinc and copper on populations of the ciliated protozoan Tetrahymena thermophila, Chemosphere, 68, 647-661, doi:10.1016/j.chemosphere.2007.02.031, 2007.

Garbe-Schönberg, D. and Müller, S.: Nano-particulate pressed powder tablets for LA-ICP-MS, Journal of Analytical Atomic Spectrometry, 29, 990-1000, doi:10.1039/C4JA00007B, 2014.

Geisler, C.-D. and Schmidt, D.: An overview of chromium in the marine environment, Deutsche Hydrografische Zeitschrift, 44, 185-196, doi:10.15835/BUASVMCN-VM:63:1-2:2516, 1991.

Glas, M. S., Langer, G., and Keul, N.: Calcification acidifies the microenvironment of a benthic foraminifer (Ammonia sp.), Journal of Experimental Marine Biology and Ecology, 424, 53-58, doi:10.1016/j.jembe.2012.05.006, 2012.

Gooday, A. J.: A response by benthic foraminifera to the deposition of phytodetritus in the deep sea, Nature, 332 , 70-73, doi:10.1038/332070a0, 1988

Groeneveld, J. and Filipsson, H. L.: $\mathrm{Mg} / \mathrm{Ca}$ and $\mathrm{Mn} / \mathrm{Ca}$ ratios in benthic foraminifera: The potential to reconstruct past variations in temperature and hypoxia in shelf regions, Biogeosciences, 10, 5125-5138, doi:10.5194/bg$10-5125-2013,2013$.

Haake, F. W.: Zum Jahresgang von Populationen einer Foraminiferen-Art in der westlichen Ostsee, Meyniana, 17, 13-27, 1967

Haake, F.-W.: Untersuchungen an der Foraminiferen-Fauna im Wattgebiet zwischen Langeoog und dem Festland, Meyniana, 12, 25-64, 1962.

Haley, B. A., Klinkhammer, G. P., and Mix, A. C.: Revisiting the rare earth elements in foraminiferal tests, Earth and Planetary Science Letters, 239, 79-97, doi:10.1016/j.eps1.2005.08.014, 2005.

Hall, J. M. and Chan, L.-H.: Li/Ca in multiple species of benthic and planktonic foraminifera: Thermocline, latitudinal, and glacial-interglacial variation, Geochimica et Cosmochimica Acta, 68, 529-545, doi:10.1016/S0016-7037(03)00451-4, 2004.

Hammer, Ø., Harper, D. A. T., and Ryan, P. D.: PAST: Paleontological statistics software package for education and data analysis, Palaeontologia Electronica, 4, 9, 2001.

Hänsch, R. and Mendel, R. R.: Physiological functions of mineral micronutrients (Cu, Zn, Mn, Fe, Ni, Mo, B, cl), Current Opinion in Plant Biology, 12, 259-266, doi:10.1016/j.pbi.2009.05.006, 2009.

Havach, S. M., Chandler, G. T., Wilson-Finelli, A., and Shaw, T. J.: Experimental determination of trace element partition coefficients in cultured benthic foraminifera, Geochimica et Cosmochimica Acta, 65, 1277-1283, doi:10.1016/S0016-7037(00)00563-9, 2001.

Haynert, K., Schönfeld, J., Riebesell, U., and Polovodova, I.: Biometry and dissolution features of the benthic foraminifer Ammonia aomoriensis at high $\mathrm{pCO}_{2}$, Marine Ecology Progress Series, 432, 53-67, doi:10.3354/meps09138, 2011.

Hintz, C. J., Chandler, G. T., Bernhard, J. M., McCorkle, D. C., Havach, S. M., Blanks, J. K., and Shaw, T. J.: A physicochemically constrained seawater culturing system for production of benthic foraminifera, Limnology and Oceanography: Methods, 2, 160-170, doi:10.4319/lom.2004.2.160, 2004. 
Horovitz, C. T.: Is the major part of the periodic system really inessential for life?, Journal of Trace Elements and Electrolytes in Health and Disease, 2, 135-144, 1988.

Huang, H., Yuan, X., Zeng, G., Zhu, H., Li, H., Liu, Z., Jiang, H., Leng, L., and Bi, W.: Quantitative evaluation of heavy metals' pollution hazards in liquefaction residues of sewage sludge, Bioresource Technology, 102, 10346-10351, doi:10.1016/j.biortech.2011.08.117, 2011.

Huang, J., Yuan, F., Zeng, G., Li, X., Gu, Y., Shi, L., Liu, W., and Shi, Y.: Influence of pH on heavy metal speciation and removal from wastewater using micellar-enhanced ultrafiltration, Chemosphere, 173, 199-206, doi:10.1016/j.chemosphere.2016.12.137, 2017.

Inoue, M., Nohara, M., Okai, T., Suzuki, A., and Kawahata, H.: Concentrations of trace elements in carbonate reference materials coral JCp-1 and giant clam JCt-1 by inductively coupled plasma-mass spectrometry, Geostandards and Geoanalytical Research, 28, 411-416, doi:10.1111/j.1751-908X.2004.tb00759.x, 2004.

Ishikawa, M. and Ichikuni, M.: Uptake of sodium and potassium by calcite, Chemical Geology, 42, 137-146, doi:10.1016/0009-2541(84)90010-X, 1984.

Jan, A. T., Azam, M., Siddiqui, K., Ali, A., Choi, I., and Haq, Q. M.: Heavy metals and human health: Mechanistic insight into toxicity and counter defense system of antioxidants, International Journal of Molecular Sciences, 16, 29592-29630, doi:10.3390/ijms161226183, 2015.

Jauffrais, T., Jesus, B., Metzger, E., Mouget, J.-L., Jorissen, F., and Geslin, E.: Effect of light on photosynthetic efficiency of sequestered chloroplasts in intertidal benthic foraminifera (Haynesina germanica and Ammonia tepida), Biogeosciences, 13, 2715-2726, doi:10.5194/bg-13-2715-2016, 2016.

Jochum, K. P., Garbe-Schönberg, D., Veter, M., Stoll, B., Weis, U., Weber, M., Lugli, F., Jentzen, A., Schiebel, R., and Wassenburg, J. A.: Nano-powdered calcium carbonate reference materials: Significant progress for microanalysis?, Geostandards and Geoanalytical Research, 43, 595-609, doi:10.1111/ggr.12292, 2019.

Jochum, K. P., Weis, U., Stoll, B., Kuzmin, D., Yang, Q., Raczek, I., Jacob, D. E., Stracke, A., Birbaum, K., and Frick, D. A.: Determination of reference values for NIST SRM 610-617 glasses following ISO guidelines, Geostandards and Geoanalytical Research, 35, 397-429, doi:10.1111/j.1751-908X.2011.00120.x, 2011.

Julian, P.: South Florida Coastal Sediment Ecological Risk Assessment, Bulletin of Environmental Contamination and Toxicology, 95, 188-193, doi:10.1007/s00128-015-1583-8, 2015.

Kannan, K., Smith Jr, R. G., Lee, R. F., Windom, H. L., Heitmuller, P. T., Macauley, J. M., and Summers, J. K.: Distribution of total mercury and methyl mercury in water, sediment, and fish from south Florida estuaries, Archives of Environmental Contamination and Toxicology, 34, 109-118, doi:10.1007/s002449900294, 1998. Kennish, M. J.: Ecology of estuaries: Anthropogenic effects, CRC press, Vol. 1, doi:10.2307/1351438, 2019.

Khalifa, G. M., Kirchenbuechler, D., Koifman, N., Kleinerman, O., Talmon, Y., Elbaum, M., Addadi, L., Weiner, S., and Erez, J.: Biomineralization pathways in a foraminifer revealed using a novel correlative cryofluorescence-SEM-EDS technique, Journal of Structural Biology, 196, 155-163, doi:10.1016/j.jsb.2016.01.015, 2016.

Koho, K. A., Nooijer, L. J. de, Fontanier, C., Toyofuku, T., Oguri, K., Kitazato, H., and Reichart, G.-J.: Benthic foraminiferal $\mathrm{Mn} / \mathrm{Ca}$ ratios reflect microhabitat preferences, Biogeosciences, 14, 3067-3082, doi:10.5194/bg14-3067-2017, 2017.

Koho, K. A., Nooijer, L. J. de, and Reichart, G. J.: Combining benthic foraminiferal ecology and shell Mn/Ca to deconvolve past bottom water oxygenation and paleoproductivity, Geochimica et Cosmochimica Acta, 165, 294-306, doi:10.1016/j.gca.2015.06.003, 2015. 
Kotthoff, U., Groeneveld, J., Ash, J. L., Fanget, A.-S., Krupinski, N. Q., Peyron, O., Stepanova, A., Warnock, J., van Helmond, N. A., and Passey, B. H.: Reconstructing Holocene temperature and salinity variations in the western Baltic Sea region: A multi-proxy comparison from the Little Belt (IODP Expedition 347, Site M0059), Biogeosciences, 14, 5607-5632, doi:10.5194/bg-14-5607-2017, 2017.

Le Cadre, V. and Debenay, J.-P.: Morphological and cytological responses of Ammonia (foraminifera) to copper contamination: Implication for the use of foraminifera as bioindicators of pollution, Environmental Pollution, 143, 304-317, doi:10.1016/j.envpol.2005.11.033, 2006.

Lee, J. J., Muller, W. A., Stone, R. J., McEnery, M. E., and Zucker, W.: Standing crop of foraminifera in sublittoral epiphytic communities of a Long Island salt marsh, Marine Biology, 4, 44-61, doi:10.1007/BF00372165, 1969.

Leonhard, P., Pepelnik, R., Prange, A., Yamada, N., and Yamada, T.: Analysis of diluted sea-water at the ng L ${ }^{-1}$ level using an ICP-MS with an octopole reaction cell, Journal of Analytical Atomic Spectrometry, 17, 189196, doi:10.1039/B110180N, 2002.

Li, H., Lin, L., Ye, S., Li, H., and Fan, J.: Assessment of nutrient and heavy metal contamination in the seawater and sediment of Yalujiang Estuary, Marine Pollution Bulletin, 117, 499-506, doi:10.1016/j.marpolbul.2017.01.069, 2017.

Linshy, V. N., Saraswat, R., Kurtarkar, S. R., and Nigam, R.: Experiment to decipher the effect of heavy metal cadmium on coastal benthic foraminifer Pararotalia nipponica (Asano), Journal of the Palaeontological Society of India, 205-211, 2013.

Losada Ros, M. T., Al-Enezi, E., Cesarini, E., Canonico, B., Bucci, C., Alves Martins, M. V., Papa, S., and Frontalini, F.: Assessing the Cadmium Effects on the Benthic Foraminifer Ammonia cf. parkinsoniana: An Acute Toxicity Test, Water, 12, 1018, doi:10.3390/w12041018, 2020.

Lukaski, H. C.: Chromium as a supplement, Annual Review of Nutrition, 19, 279-302, doi:10.1146/annurev.nutr.19.1.279, 1999.

Marchitto Jr, T. M., Curry, W. B., and Oppo, D. W.: Zinc concentrations in benthic foraminifera reflect seawater chemistry, Paleoceanography, 15, 299-306, doi:10.1029/1999PA000420, 2000.

Maréchal-Abram, N., Debenay, J.-P., Kitazato, H., and Wada, H.: Cadmium partition coefficients of cultured benthic foraminifera Ammonia beccarii, Geochemical Journal, 38, 271-283, doi:10.2343/geochemj.38.271, 2004.

Maret, W.: The metals in the biological periodic system of the elements: Concepts and conjectures, International Journal of Molecular Sciences, 17, 66, doi:10.3390/ijms17010066, 2016.

Martelli, A., Rousselet, E., Dycke, C., Bouron, A., and Moulis, J.-M.: Cadmium toxicity in animal cells by interference with essential metals, Biochimie, 88, 1807-1814, doi:10.1016/j.biochi.2006.05.013, 2006.

Martin, P. A. and Lea, D. W.: A simple evaluation of cleaning procedures on fossil benthic foraminiferal $\mathrm{Mg} / \mathrm{Ca}$, Geochemistry, Geophysics, Geosystems, 3, 1-8, doi:10.1029/2001GC000280, 2002.

Martinez-Colon, M., Hallock, P., and Green-Ruíz, C.: Strategies for using shallow-water benthic foraminifers as bioindicators of potentially toxic elements: A review, Journal of Foraminiferal Research, 39, 278-299, doi:10.2113/gsjfr.39.4.278, 2009.

Martinez-Finley, E. J., Chakraborty, S., Fretham, S. J. B., and Aschner, M.: Cellular transport and homeostasis of essential and nonessential metals, Metallomics, 4, 593-605, doi:10.1039/c2mt00185c, 2012. 
Martín-González, A., Borniquel, S., Díaz, S., Ortega, R., and Gutiérrez, J. C.: Ultrastructural alterations in ciliated protozoa under heavy metal exposure, Cell Biology International, 29, 119-126, doi:10.1016/j.cellbi.2004.09.010, 2005.

McCorkle, D. C., Martin, P. A., Lea, D. W., and Klinkhammer, G. P.: Evidence of a dissolution effect on benthic foraminiferal shell chemistry: $\delta^{13} \mathrm{C}, \mathrm{Cd} / \mathrm{Ca}, \mathrm{Ba} / \mathrm{Ca}$, and $\mathrm{Sr} / \mathrm{Ca}$ results from the Ontong Java Plateau, Paleoceanography, 10, 699-714, doi:10.1029/95PA01427, 1995.

McGann, M.: High-resolution foraminiferal, isotopic, and trace element records from Holocene estuarine deposits of San Francisco Bay, California, Journal of Coastal Research, 24, 1092-1109, doi:10.2112/08A-0003.1, 2008

Mertz, W.: The essential trace elements, Science, 213, 1332-1338, doi:10.1126/science.7022654, 1981.

Mertz, W.: Chromium in human nutrition: A review, The Journal of Nutrition, 123, 626-633, doi:10.1093/jn/123.4.626, 1993.

Millero, F. J., Woosley, R., Ditrolio, B., and Waters Jason: Effect of ocean acidification on the speciation of metals in seawater, Oceanography, 22, 72-85, doi:10.5670/oceanog.2009.98, 2009.

Munsel, D., Kramar, U., Dissard, D., Nehrke, G., Berner, Z., Bijma, J., Reichart, G.-J., and Neumann, T.: Heavy metal uptake in foraminiferal calcite: Results of multi-element culture experiments, Biogeosciences Discussions, 7, doi:10.5194/bg-7-2339-2010, 2010.

Murray, J. W.: Ecology and palaeoecology of benthic foraminifera: Longman Scientific and Technical, Harlow, Essex, UK, doi:10.4324/9781315846101, 1991.

Murray, J. W.: Distribution and population dynamics of benthic foraminifera from the southern North Sea, Journal of Foraminiferal Research, 22, 114-128, doi:10.2113/gsjfr.22.2.114, 1992.

Muse, J. O., Stripeikis, J. D., Fernandez, F. M., d'Huicque, L., Tudino, M. B., Carducci, C. N., and Troccoli, O. E.: Seaweeds in the assessment of heavy metal pollution in the Gulf San Jorge, Argentina, Environmental Pollution, 104, 315-322, doi:10.1016/S0269-7491(98)00096-7, 1999.

Mutwakil, M., Reader, J. P., Holdich, D. M., Smithurst, P. R., Candido, E. P.M., Jones, D., Stringham, E. G., and de Di Pomerai: Use of stress-inducible transgenic nematodes as biomarkers of heavy metal pollution in water samples from an English river system, Archives of Environmental Contamination and Toxicology, 32, 146153, doi:10.1007/s002449900167, 1997.

Nardelli, M. P., Malferrari, D., Ferretti, A., Bartolini, A., Sabbatini, A., and Negri, A.: Zinc incorporation in the miliolid foraminifer Pseudotriloculina rotunda under laboratory conditions, Marine Micropaleontology, 126, 42-49, doi:10.1016/j.marmicro.2016.06.001, 2016.

Nehrke, G., Keul, N., Langer, G., Nooijer, L. J. de, Bijma, J., and Meibom, A.: A new model for biomineralization and trace-element signatures of Foraminifera tests, Biogeosciences, 10, 6759-6767, doi:10.5194/bg-10-67592013, 2013

Nooijer, L. J. de, Langer, G., Nehrke, G., and Bijma, J.: Physiological controls on seawater uptake and calcification in the benthic foraminifer Ammonia tepida, Biogeosciences, 6, 2669-2675, doi:10.5194/bg-6-2669-2009, 2009a.

Nooijer, L. J. de, Reichart, G.-J., Dueñas Bohórquez, A. D.B., Wolthers, M., Ernst, SR, Mason, P. R.D., and van der Zwaan, G. J.: Copper incorporation in foraminiferal calcite: Results from culturing experiments, Biogeosciences Discussions, 4, 961-991, doi:10.5194/bg-4-493-2007, 2007. 
Nooijer, L. J. de, Spero, H. J., Erez, J., Bijma, J., and Reichart, G.-J.: Biomineralization in perforate foraminifera, Earth-Science Reviews, 135, 48-58, doi:10.1016/j.earscirev.2014.03.013, 2014.

Nooijer, L. J. de, Toyofuku, T., and Kitazato, H.: Foraminifera promote calcification by elevating their intracellular pH, Proceedings of the National Academy of Sciences, 106, 15374-15378, doi:10.1073/pnas.0904306106, 2009b.

Nordberg, G. F., Jin, T., Hong, F., Zhang, A., Buchet, J.-P., and Bernard, A.: Biomarkers of cadmium and arsenic interactions, Toxicology and Applied Pharmacology, 206, 191-197, doi:10.1016/j.taap.2004.11.028, 2005.

Nürnberg, D.: Magnesium in tests of Neogloboquadrina pachyderma sinistral from high northern and southern latitudes, Journal of Foraminiferal Research, 25, 350-368, doi:10.2113/gsjfr.25.4.350, 1995.

Nürnberg, D., Bijma, J., and Hemleben, C.: Assessing the reliability of magnesium in foraminiferal calcite as a proxy for water mass temperatures, Geochimica et Cosmochimica Acta, 60, 803-814, doi:10.1016/00167037(95)00446-7, 1996.

Okumura, M. and Kitano, Y.: Coprecipitation of alkali metal ions with calcium carbonate, Geochimica et Cosmochimica Acta, 50, 49-58, doi:10.1016/0016-7037(86)90047-5, 1986.

Pagnanelli, F., Esposito, A., Toro, L., and Veglio, F.: Metal speciation and pH effect on $\mathrm{Pb}, \mathrm{Cu}, \mathrm{Zn}$ and $\mathrm{Cd}$ biosorption onto Sphaerotilus natans: Langmuir-type empirical model, Water Research, 37, 627-633, doi:10.1016/S0043-1354(02)00358-5, 2003.

Paton, C., Hellstrom, J., Paul, B., Woodhead, J., and Hergt, J.: Iolite: Freeware for the visualisation and processing of mass spectrometric data, Journal of Analytical Atomic Spectrometry, 26, 2508-2518, doi:10.1039/C1JA10172B, 2011.

Petersen, J., Barras, C., Bézos, A., La, C., Nooijer, L. J. de, Meysman, F., JR, Mouret, A., Slomp, C. P., and Jorissen, F. J.: $\mathrm{Mr} / \mathrm{Ca}$ intra-and inter-test variability in the benthic foraminifer Ammonia tepida, Biogeosciences, 15, 331-348, doi:10.5194/bg-15-331-2018, 2018.

Pillet, L., Vargas, C. de, and Pawlowski, J.: Molecular identification of sequestered diatom chloroplasts and kleptoplastidy in foraminifera, Protist, 162, 394-404, doi:10.1016/j.protis.2010.10.001, 2011.

Pilon-Smits, E. A. H., Quinn, C. F., Tapken, W., Malagoli, M., and Schiavon, M.: Physiological functions of beneficial elements, Current Opinion in Plant Biology, 12, 267-274, doi:10.1016/j.pbi.2009.04.009, 2009.

Platon, E., Gupta, B. K. S., Rabalais, N. N., and Turner, R. E.: Effect of seasonal hypoxia on the benthic foraminiferal community of the Louisiana inner continental shelf: The $20^{\text {th }}$ century record, Marine Micropaleontology, 54, 263-283, doi:10.1016/j.marmicro.2004.12.004, 2005.

Poignant, A., Mathieu, R., Levy, A., and Cahuzac, B.: Haynesina germanica (Ehrenberg), Elphidium excavatum (Terquem) ls et Porosononion granosum (d'Orbigny), espèces margino-littorales de foraminiféres d'Aquitaine centrale (SO France) au Miocène Moyen (Langhien). Le problème d'Elphidium lidoense Cushman, Revue de Micropaléontologie, 43, 393-405, doi:10.1016/S0035-1598(00)90200-9, 2000.

Polovodova, I. and Schönfeld, J.: Foraminiferal test abnormalities in the western Baltic Sea, Journal of Foraminiferal Research, 38, 318-336, doi:10.2113/gsjfr.38.4.318, 2008.

Poonkothai, M. and Vijayavathi, B. S.: Nickel as an essential element and a toxicant, International Journal of Environmental Sciences, 1, 285-288, 2012.

Powell, K. J., Brown, P. L., Byrne, R. H., Gajda, T., Hefter, G., Leuz, A.-K., Sjöberg, S., and Wanner, H.: Chemical Speciation of Environmentally Significant Metals: An IUPAC contribution to reliable and rigorous computer modelling, Chemistry International, 37, 15-19, doi:10.1515/ci-2015-0105, 2015. 
Prothro, M. G.: Office of Water policy and technical guidance on interpretation and implementation of aquatic life metals criteria, United States Environmental Protection Agency., 1993.

Putri, L. S. E., Prasetyo, A. D., and Arifin, Z.: Green mussel (Perna viridis L.) as bioindicator of heavy metals pollution at Kamal estuary, Jakarta Bay, Indonesia, Journal of Environmental Research and Development, 6, 389-396, 2012.

Raikwar, M. K., Kumar, P., Singh, M., and Singh, A.: Toxic effect of heavy metals in livestock health, Veterinary World, 1, 28, doi:10.5455/vetworld.2008.28-30, 2008.

Railsback, L. B.: Patterns in the compositions, properties, and geochemistry of carbonate minerals, Carbonates and Evaporites, 14, 1, doi:10.1007/BF03176144, 1999.

Ramasamy, E. V., Jayasooryan, K. K., Chandran, M. S., and Mohan, M.: Total and methyl mercury in the water, sediment, and fishes of Vembanad, a tropical backwater system in India, Environmental Monitoring and Assessment, 189, 130, doi:10.1007/s10661-017-5845-2, 2017.

Reddy, M. S., Basha, S., Joshi, H. V., and Ramachandraiah, G.: Seasonal distribution and contamination levels of total PHCs, PAHs and heavy metals in coastal waters of the Alang-Sosiya ship scrapping yard, Gulf of Cambay, India, Chemosphere, 61, 1587-1593, doi:10.1016/j.chemosphere.2005.04.093, 2005.

Reeder, R. J., Lamble, G. M., and Northrup, P. A.: XAFS study of the coordination and local relaxation around $\mathrm{Co}^{2} \mathrm{Zn}^{2+}, \mathrm{Pb}^{2+}$, and $\mathrm{Ba}^{2+}$ trace elements in calcite, American Mineralogist, 84, 1049-1060, doi:10.2138/am1999-7-807, 1999.

Remmelzwaal, S. R. C., Sadekov, A. Y., Parkinson, I. J., Schmidt, D. N., Titelboim, D., Abramovich, S., Roepert, A., Kienhuis, M., Polerecky, L., and Goring-Harford, H.: Post-depositional overprinting of chromium in foraminifera, Earth and Planetary Science Letters, 515, 100-111, doi:10.1016/j.eps1.2019.03.001, 2019.

Rimstidt, J. D., Balog, A., and Webb, J.: Distribution of trace elements between carbonate minerals and aqueous solutions, Geochimica et Cosmochimica Acta, 62, 1851-1863, doi:10.1016/S0016-7037(98)00125-2, 1998.

Roberts, N. L., Piotrowski, A. M., Elderfield, H., Eglinton, T. I., and Lomas, M. W.: Rare earth element association with foraminifera, Geochimica et Cosmochimica Acta, 94, 57-71, doi:10.1016/j.gca.2012.07.009, 2012.

Rosenthal, Y., Boyle, E. A., and Slowey, N.: Temperature control on the incorporation of magnesium, strontium, fluorine, and cadmium into benthic foraminiferal shells from Little Bahama Bank: Prospects for thermocline paleoceanography, Geochimica et Cosmochimica Acta, 61, 3633-3643, doi:10.1016/S0016-7037(97)001816, 1997.

Rosenthal, Y., Field, M. P., and Sherrell, R. M.: Precise determination of element/calcium ratios in calcareous samples using sector field inductively coupled plasma mass spectrometry, Analytical Chemistry, 71, 32483253, doi:10.1021/ac981410x, 1999.

Sagar, N., Sadekov, A., Scott, P., Jenner, T., Vadiveloo, A., Moheimani, N. R., and McCulloch, M.: Geochemistry of large benthic foraminifera Amphisorus hemprichii as a high-resolution proxy for lead pollution in coastal environments, Marine Pollution Bulletin, 162, 111918, doi:10.1016/j.marpolbul.2020.111918, 2021.

Saha, N., Rahman, M. S., Ahmed, M. B., Zhou, J. L., Ngo, H. H., and Guo, W.: Industrial metal pollution in water and probabilistic assessment of human health risk, Journal of Environmental Management, 185, 70-78, doi:10.1016/j.jenvman.2016.10.023, 2017.

Schlitzer, R.: Ocean Data View, http://odv.awi.de, 2016.

Schönfeld, J. and Numberger, L.: The benthic foraminiferal response to the 2004 spring bloom in the western Baltic Sea, Marine Micropaleontology, 65, 78-95, doi:10.1016/j.marmicro.2007.06.003, 2007. 
Sen Gupta, B. K., Eugene Turner, R., and Rabalais, N. N.: Seasonal oxygen depletion in continental-shelf waters of Louisiana: Historical record of benthic foraminifers, Geology, 24, 227-230, doi:10.1130/00917613(1996)024<0227:SODICS>2.3.CO;2, 1996.

Shannon, R. T. D. and Prewitt, C. T.: Effective ionic radii in oxides and fluorides, Acta Crystallographica Section B: Structural Crystallography and Crystal Chemistry, 25, 925-946, doi:10.1107/S0567740869003220, 1969.

Sharifi, A. R., Croudace, I. W., and Austin, R. L.: Benthic foraminiferids as pollution indicators in Southampton Water, southern England, UK, Journal of Micropalaeontology, 10, 109-113, doi:10.1144/jm.10.1.109, 1991.

Shaw, D. R. and Dussan, J.: Mathematical modelling of toxic metal uptake and efflux pump in metal-resistant bacterium Bacillus cereus isolated from heavy crude oil, Water, Air, \& Soil Pollution, 226, 1-14, doi:10.1007/s11270-015-2385-7, 2015.

Shijo, Y., Shimizu, T., and Tsunoda, T.: Determination of silver in seawater by graphite furnace atomic absorption spectrometry after solvent extraction and microscale back-extraction, Analytical Sciences, 5, 65-68, doi:10.2116/analsci.5.65, 1989.

Smith, C. W., Fehrenbacher, J. S., and Goldstein, S. T.: Incorporation of heavy metals in experimentally grown foraminifera from SAPELO island, Georgia and little duck key, Florida, USA, Marine Micropaleontology, 101854, doi:10.1016/j.marmicro.2020.101854, 2020.

Spindler, M.: The development of the organic lining in Heterostegina depressa (Nummulitidae; Foraminifera), Journal of Foraminiferal Research, 8, 258-261, doi:10.2113/gsjfr.8.3.258, 1978.

Spurgeon, D. J., Lofts, S., Hankard, P. K., Toal, M., McLellan, D., Fishwick, S., and Svendsen, C.: Effect of pH on metal speciation and resulting metal uptake and toxicity for earthworms, Environmental Toxicology and Chemistry: An International Journal, 25, 788-796, doi:10.1897/05-045R1.1, 2006.

Stankovic, S., Kalaba, P., and Stankovic, A. R.: Biota as toxic metal indicators, Environmental Chemistry Letters, 12, 63-84, doi:10.1007/s10311-013-0430-6, 2014.

Sunda, W. G. and Huntsman, S. A.: Interactions among $\mathrm{Cu}^{2+}, \mathrm{Zn}^{2+}$, and $\mathrm{Mn}^{2+}$ in controlling cellular $\mathrm{Mn}, \mathrm{Zn}$, and growth rate in the coastal alga Chlamydomonas, Limnology and Oceanography, 43, 1055-1064, doi:10.4319/lo.1998.43.6.1055, 1998a

Sunda, W. G. and Huntsman, S. A.: Processes regulating cellular metal accumulation and physiological effects: Phytoplankton as model systems, Science of the Total Environment, 219, 165-181, doi:10.1016/S00489697(98)00226-5, 1998b.

Tachikawa, K. and Elderfield, H.: Microhabitat effects on $\mathrm{Cd} / \mathrm{Ca}$ and $\delta^{13} \mathrm{C}$ of benthic foraminifera, Earth and Planetary Science Letters, 202, 607-624, doi:10.1016/S0012-821X(02)00796-3, 2002.

Tansel, B. and Rafiuddin, S.: Heavy metal content in relation to particle size and organic content of surficial sediments in Miami River and transport potential, International Journal of Sediment Research, 31, 324-329, doi:10.1016/j.ijsrc.2016.05.004, 2016.

Tchounwou, P. B., Yedjou, C. G., Patlolla, A. K., and Sutton, D. J.: Heavy metal toxicity and the environment, Molecular, clinical and environmental toxicology, 133-164, doi:10.1007/978-3-7643-8340-4_6, 2012.

Thomas, M. A., Conaway, C. H., Steding, D. J., Marvin-DiPasquale, M., Abu-Saba, K. E., and Flegal, A. R.: Mercury contamination from historic mining in water and sediment, Guadalupe River and San Francisco Bay, California, Geochemistry: Exploration, Environment, Analysis, 2, 211-217, doi:10.1144/1467-787302-024, 2002. 
Titelboim, D., Sadekov, A., Hyams-Kaphzan, O., Almogi-Labin, A., Herut, B., Kucera, M., and Abramovich, S.: Foraminiferal single chamber analyses of heavy metals as a tool for monitoring permanent and short term anthropogenic footprints, Marine Pollution Bulletin, 128, 65-71, doi:10.1016/j.marpolbul.2018.01.002, 2018.

Toyofuku, T., Suzuki, M., Suga, H., Sakai, S., Suzuki, A., Ishikawa, T., Nooijer, L. J. de, Schiebel, R., Kawahata, H., and Kitazato, $\mathrm{H} .: \mathrm{Mg} / \mathrm{Ca}$ and $\delta^{18} \mathrm{O}$ in the brackish shallow-water benthic foraminifer Ammonia 'beccarii', Marine Micropaleontology, 78, 113-120, doi:10.1016/j.marmicro.2010.11.003, 2011.

Urani, C., Melchioretto, P., Bruschi, M., Fabbri, M., Sacco, M. G., and Gribaldo, L.: Impact of cadmium on intracellular zinc levels in HepG2 cells: Quantitative evaluations and molecular effects, BioMed Research International, 2015, doi:10.1155/2015/949514, 2015.

van Dijk, I., Nooijer, L. J. de, and Reichart, G.-J.: Trends in element incorporation in hyaline and porcelaneous foraminifera as a function of $\mathrm{pCO}_{2}$, Biogeosciences, 14, 497-510, doi:10.5194/bg-14-497-2017, 2017.

Vlahogianni, T., Dassenakis, M., Scoullos, M. J., and Valavanidis, A.: Integrated use of biomarkers (superoxide dismutase, catalase and lipid peroxidation) in mussels Mytilus galloprovincialis for assessing heavy metals' pollution in coastal areas from the Saronikos Gulf of Greece, Marine Pollution Bulletin, 54, 1361-1371, doi:10.1016/j.marpolbul.2007.05.018, 2007.

Wang, G. and Fowler, B. A.: Roles of biomarkers in evaluating interactions among mixtures of lead, cadmium and arsenic, Toxicology and Applied Pharmacology, 233, 92-99, doi:10.1016/j.taap.2008.01.017, 2008.

Wefer, G.: Umwelt, Produktion und Sedimentation benthischer Foraminiferen in der westlichen Ostsee, Reports Sonderforschungsbereich 95 Wechselwirkung Meer - Meeresboden, 14, 1-103, 1976.

Williams, T. M., Rees, J. G., and Setiapermana, D.: Metals and trace organic compounds in sediments and waters of Jakarta Bay and the Pulau Seribu Complex, Indonesia, Marine Pollution Bulletin, 40, 277-285, doi:10.1016/S0025-326X(99)00226-X, 2000.

Wit, J. C., Nooijer, L. J. de, Wolthers, M., and Reichart, G.-J.: A novel salinity proxy based on Na incorporation into foraminiferal calcite, Biogeosciences, 10, 6375-6387, doi:10.5194/bg-10-6375-2013, 2013.

Woehle, C., Roy, A.-S., Glock, N., Wein, T., Weissenbach, J., Rosenstiel, P., Hiebenthal, C., Michels, J., Schönfeld, J., and Dagan, T.: A novel eukaryotic denitrification pathway in foraminifera, Current Biology, 28, 2536-2543. e5, doi:10.1016/j.cub.2018.06.027, 2018.

Wokhe, T. B.: Heavy metals pollution of water and sediment in Mada River, Nigeria, Journal of Scientific Research and Reports, 157-164, doi:10.9734/JSRR/2015/14803, 2015.

Xiang, R., Yang, Z., Saito, Y., Fan, D., Chen, M., Guo, Z., and Chen, Z.: Paleoenvironmental changes during the last 8400 years in the southern Yellow Sea: Benthic foraminiferal and stable isotopic evidence, Marine Micropaleontology, 67, 104-119, doi:10.1016/j.marmicro.2007.11.002, 2008.

Yanko, V., Ahmad, M., and Kaminski, M.: Morphological deformities of benthic foraminiferal tests in response to pollution by heavy metals; implications for pollution monitoring, Journal of Foraminiferal Research, 28, 177-200, 1998.

Yeghicheyan, D., Aubert, D., Bouhnik-le Coz, M., Chmeleff, J., Delpoux, S., Djouraev, I., Granier, G., Lacan, F., Piro, J.-L., and Rousseau, T.: A New Interlaboratory Characterisation of Silicon, Rare Earth Elements and Twenty-Two Other Trace Element Concentrations in the Natural River Water Certified Reference Material SLRS-6 (NRC-CNRC), Geostandards and Geoanalytical Research, 43, 475-496, doi:10.1111/ggr.12268, 2019. 
https://doi.org/10.5194/bg-2021-158

Preprint. Discussion started: 12 August 2021

(C) Author(s) 2021. CC BY 4.0 License.

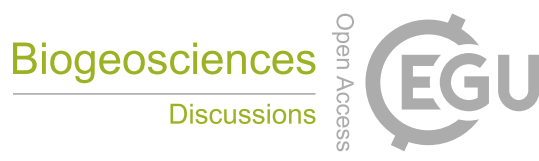

1322 Yılmaz, S. and Sadikoglu, M.: Study of heavy metal pollution in seawater of Kepez harbor of Canakkale (Turkey),

1323 Environmental Monitoring and Assessment, 173, 899-904, doi:10.1007/s10661-010-1432-5, 2011. 